\title{
STAR FORMATION IN THE CENTRAL 400 PC OF THE MILKY WAY: EVIDENCE FOR A POPULATION OF MASSIVE YOUNG STELLAR OBJECTS
}

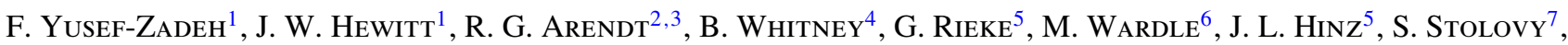 \\ C. C. LANG $^{8}$, M. G. BurTON ${ }^{9}$, AND S. RAMIREZ ${ }^{7}$ \\ ${ }^{1}$ Department of Physics and Astronomy, Northwestern University, Evanston, IL 60208, USA; zadeh@northwestern.edu \\ ${ }^{2}$ Science Systems and Applications, Inc., Baltimore County, GSFC, Code 665, Greenbelt, MD 20771, USA \\ ${ }^{3}$ University of Maryland, Baltimore County, GSFC, Code 665, Greenbelt, MD 20771, USA \\ ${ }^{4}$ Space Science Institute, 4750 Walnut Street, Suite 205, Boulder, CO 80301, USA \\ ${ }^{5}$ Steward Observatory, University of Arizona, 933 North Cherry Avenue, Tucson, AZ 85721, USA \\ ${ }^{6}$ Department of Physics and Engineering, Macquarie University, Sydney, NSW 2109, Australia \\ ${ }^{7}$ Spitzer Science Center, California Institute of Technology, Mail Code 220-6, 1200 East California Boulevard, Pasadena, CA 91125, USA \\ ${ }^{8}$ Department of Physics \& Astronomy, University of Iowa, Iowa City, IA 52245, USA \\ ${ }^{9}$ School of Physics, University of New South Wales, Sydney, NSW 2052, Australia \\ Received 2009 April 17; accepted 2009 June 16; published 2009 August 10
}

\begin{abstract}
The central kpc of the Milky Way might be expected to differ significantly from the rest of the Galaxy with regard to gasdynamics and the formation of young stellar objects (YSOs). We probe this possibility with midinfrared observations obtained with Infrared Array Camera and Multiband Imaging Photometer on Spitzer and with Midcourse Space Experiment. We use color-color diagrams and spectral energy distribution (SED) fits to explore the nature of YSO candidates (including objects with $4.5 \mu \mathrm{m}$ excesses possibly due to molecular emission). There is an asymmetry in the distribution of the candidate YSOs, which tend to be found at negative Galactic longitudes; this behavior contrasts with that of the molecular gas, approximately $2 / 3$ of which is at positive longitudes. The small-scale height of these objects suggests that they are within the Galactic center region and are dynamically young. They lie between two layers of infrared dark clouds and may have originated from these clouds. We identify new sites for this recent star formation by comparing the mid-IR, radio, submillimeter, and methanol maser data. The methanol masers appear to be associated with young, embedded YSOs characterized by $4.5 \mu \mathrm{m}$ excesses. We use the SEDs of these sources to estimate their physical characteristics; their masses appear to range from $\sim 10$ to $\sim 20 M_{\odot}$. Within the central $400 \times 50 \mathrm{pc}\left(|l|<1.3\right.$ and $\left.|b|<10^{\prime}\right)$ the star formation rate (SFR) based on the identification of Stage I evolutionary phase of YSO candidates is about $0.14 M_{\odot} \mathrm{yr}^{-1}$. Given that the majority of the sources in the population of YSOs are classified as Stage I objects, we suggest that a recent burst of star formation took place within the last $10^{5} \mathrm{yr}$. This suggestion is also consistent with estimates of SFRs within the last $\sim 10^{7} \mathrm{yr}$ showing a peak around $10^{5} \mathrm{yr}$ ago. Lastly, we find that the Schmidt-Kennicutt Law applies well in the central $400 \mathrm{pc}$ of the Galaxy. This implies that star formation does not appear to be dramatically affected by the extreme physical conditions in the Galactic center region.
\end{abstract}

Key words: galaxies: starburst - Galaxy: center - ISM: clouds - masers - stars: formation

Online-only material: color figures

\section{INTRODUCTION}

Understanding the processes occurring in the nuclear disk of our own Galaxy is interesting not only for insight into our own Milky Way Galaxy, but also because its study can potentially provide a template to the closest galactic nuclei. This important region of the Galaxy hosts several sources of energetic activity, and is the site of massive molecular clouds with pockets of past and present massive star formation. On a larger scale, the distribution of molecular clouds is asymmetric in the so-called "central molecular zone" showing $2 / 3$ of the molecular gas on the positive longitude side of the Galactic center region (Bania 1977; Bally et al. 1988; Oka et al. 2005; Tsuboi et al. 1999; Martin et al. 2004; Morris \& Serabyn 1996). The nature of this asymmetry in the context of gasdynamics is not understood. This unique region is also distinguished from the rest of the Galaxy by the presence of a bar that is responsible for feeding gas into the central few hundred parsecs of the Galaxy. In particular, the noncircular motion of the gas in this region is thought to be induced by the bar's potential combined with dynamical friction leading to the migration of molecular clouds to the
Galactic center (Binney et al. 1991; Stark et al. 1991). The collision of these migrating molecular clouds with each other in a small volume of the Galactic center is thought to be the driving mechanism for star formation on a wide range of scales (Wardle \& Yusef-Zadeh 2008). Thus, it is possible that the star formation process depends on the Galactic center environment including the effects of the bar on the gasdynamics.

Although there is a high concentration of dense molecular clouds distributed in the Galactic center region, the star formation rate (SFR) has shown extreme values. On the one hand, the massive star-forming region Sgr B2 points to the closest example of starburst activity in our Galaxy. This region contains some of the most spectacular young and dense stellar clusters having an age of a few million years (Figer et al. 1999; Paumard et al. 2006; Lu et al. 2006). In the context of recently formed stars in the nuclear disk, detailed study using Infrared Space Observatory (ISO) at 7 and $15 \mu \mathrm{m}$ indicates the presence of a population of young stellar object (YSO) candidates in the region $-0.424<l<-0.115$ and $-0.194<b<0.089$ (Omont et al. 2003; Schuller et al. 2006). On the other hand, quiescent giant molecular clouds such as G0.25-0.01 (Lis \& Carlstrom 
1994; Lis \& Menten 1998) appear to contain highly inefficient star formation.

One intriguing question is whether the star formation in this region proceeds analogously to that elsewhere in the Galaxy, or whether the central region provides a unique environment and might provide a template for alternative modes of star formation in galactic nuclei generally. For example, the molecular clouds in the Galactic center region have elevated temperatures. Molecular line studies yield temperature estimates of 75-200 K (Hüttemeister et al. 1993), while far-infrared data indicate dust temperatures $\leqslant 30 \mathrm{~K}$ (Odenwald \& Fazio 1984; Cox \& Laureijs 1989; Pierce-Price et al. 2000). This temperature discrepancy suggests direct heating of the molecular gas either by cosmic rays or shocks, which may reduce the efficiency of star formation in the region (Lis et al. 2001; Yusef-Zadeh et al. 2007b).

In order to census past and present star formation activity and to gain insight into the nature of star formation in the unique environment of the Galactic center, we carried out a $24 \mu \mathrm{m}$ survey of the central molecular zone and beyond between $|l|<4^{\circ}$ and $|b|<1^{\circ}$. This is the most recent in a series of papers that have presented sensitive surveys of the Galactic center region at near-IR and mid-IR wavelengths. Our Multiband Imaging Photometer for Spitzer (MIPS) survey provides an important data point when combined with the Spitzer Infrared Array Camera (IRAC) survey of the Galactic center region (Ramirez et al. 2008; Stolovy et al. 2006; Arendt et al. 2008). This allows us to distinguish YSOs and asymptotic giant branches (AGBs) by fitting the spectral energy distribution (SED) of infrared point sources as well as by constructing color-magnitude diagrams (CMDs). Furthermore, $24 \mu \mathrm{m}$ observations reveal dense, cold dust clouds through their absorption against background emission and identify warm dust clouds through their emission without being contaminated by polycyclic aromatic hydrocarbon (PAH) emission. The presence of large-scale infrared dark clouds (IRDCs) in the Galactic center at this wavelength traces potential sites of the earliest phase of active and quiescent star formation. In particular, the correlation of $24 \mu \mathrm{m}$ point sources distributed toward IRDCs with $4.5 \mu \mathrm{m}$ excess sources, thought to trace shock excited molecular emission, can be used to identify the earliest phase of massive star formation (Chambers et al. 2009). Lastly, $24 \mu \mathrm{m}$ emission points to $\mathrm{H}$ II regions with embedded thermal sources of heating as well as to sites where synchrotron emission from relativistic particles is produced. The ratio of $24 \mu \mathrm{m}$ to radio emission can separate thermal from nonthermal sources (Fuerst et al. 1987).

The structure of this paper is as follows. Section 2 describes details of observations using MIPS, IRAC, and radio data, while Section 3 presents the large-scale distribution of extended clouds and compact stellar sources. Then in Section 4, we focus on the stellar distribution of $24 \mu \mathrm{m}$ sources and the CMD of the sources covered by the MIPS and IRAC surveys. Section 5 discusses the SEDs of candidate YSOs followed by a brief description of individual candidate sites of on-going star formation activity. The new sites of young, massive star formation are found by the detection of sources with excess $4.5 \mu \mathrm{m}$ emission. There is a rich collection of prominent infrared sources toward the Galactic center, some of which such as Sgr A, the radio Arc, and Sgr C, are saturated. We discuss elsewhere the distribution of $24 \mu \mathrm{m}$ emission from Sgr A (G0.0-0.0) and the Galactic center radio Arc (G0.2-0.0), the Sgr B H II complex (G0.7-0.0) and G0.6-0.0, Sgr C (G359.4-0.1), Sgr E (G358.7-0.0), and the Tornado nebula. Here, we assume that the distance to the Galactic center is $8.5 \mathrm{kpc}$ (Ghez et al. 2008).

\section{OBSERVATIONS AND DATA REDUCTIONS}

Apart from the $24 \mu \mathrm{m}$ survey, several other surveys of this region have recently been carried out in other wavelength bands, as described briefly below. We have correlated these data with our MIPS survey data.

\subsection{MIPS Survey at $24 \mu \mathrm{m}$}

The Galactic center region is very bright at mid-IR wavelengths. Therefore, to cover the full $8^{\circ} \times 2^{\circ}$ survey area most efficiently, we used MIPS in the fast scan mode with large crossscan steps of $302^{\prime \prime}$. Because of the permitted scan orientation during the Galactic center visibility window, the map was a parallelogram. This fast scan mode achieved enough individual source sightings for high reliability and reached the specified $1 \mathrm{mJy}$ detection limit. Eight rectangular scans were performed in sequence, each taking approximately $2.3 \mathrm{hr}$, giving a total observing time of $\sim 18 \mathrm{hr}$. The diffraction limit of the $24 \mu \mathrm{m}$ image is $5.8 \operatorname{arcsec}^{2}$. These data represent the highest spatial resolution and sensitivity large-scale map made of the inner few degrees of the Galactic center at $24 \mu \mathrm{m}$. The source photometry was based on the $24 \mu \mathrm{m}$ catalog compiled by Hinz et al. (2009).

Our data are complemented by the Spitzer Legacy program MIPS Inner Galactic Plane Survey (MIPSGAL) which observed 220 degrees $^{2}$ of the inner Galactic plane, $65^{\circ}>l>10^{\circ}$ and $-10^{\circ}>l>-65^{\circ}$ for $|b|<1^{\circ}$, at 24 and $70 \mu \mathrm{m}$ (Carey et al. 2009) which avoided duplicating coverage of the Galactic center region. MIPSGAL-II post-BCD data were used to fill gaps around the high latitude edges. This was done by reprojecting the MIPSGAL II data over the whole field. The MIPSGAL data are only used where there is zero coverage at the edges of the mosaic.

There are several $\mathrm{H}$ II regions where the $24 \mu \mathrm{m}$ emission is saturated. The most extended saturated regions lie toward Sgr A, the radio Arc, and Sgr C, near $l \sim 0^{\circ}, 0.2,0.6$, respectively. The point source saturation limit, depending on the background level, ranges between 4 and $4.5 \mathrm{Jy}$ which correspond to 0.60 and $0.51 \mathrm{mag}$ at $24 \mu \mathrm{m}$. In order to remedy the saturation artifacts, we replaced the saturated regions by Midcourse Space Experiment $(M S X)$ Band E data $(21.34 \mu \mathrm{m})$ using the following steps. (1) The $M S X$ data are reprojected to match the pixel scale and alignment of the MIPS image. A relative distortion between the positions of sources in MSX and MIPS images was corrected by a fourth-order two-dimensional polynomial warping of the $M S X$ images. This correction reduced positional differences to $\sim 0.5$ pixels ( 2 ". 49 pixels). (2) A linear fit to the correlation between pixel intensities is performed to derive a photometric scale factor and offset to be applied to match $M S X$ Band E intensities with MIPS $24 \mu \mathrm{m}$ intensities. The correlation is performed with bright (but not saturated) data by restricting pixel intensities to $>500 \mathrm{MJy} \mathrm{sr}^{-1}$ for the MIPS data and in the range of $(1-4.5) \times 10^{-5} \mathrm{~W} \mathrm{~m}^{-2} \mathrm{sr}^{-1}$. The derived scale factor was $\sim 40 \%$ larger than the quoted factor for converting $M S X$ data from $\mathrm{W} \mathrm{m} \mathrm{m}^{-2} \mathrm{sr}^{-1}$ to $\mathrm{MJy} \mathrm{sr}^{-1}$. The reason for such a discrepancy is not clear, but part of the difference may be caused by the slightly different nominal wavelengths of the instruments. (3) A mask is defined to identify the saturated regions. The mask is set to 1.0 where the rescaled $M S X$ data are brighter than $1000 \mathrm{MJy} \mathrm{sr}^{-1}$ and then ramped down to 0.0 for any pixels $>3$ pixels away from these regions. (4) The mask and 1.0 minus the mask are then applied as weights in the linear combination of the MSX and MIPS images. Thus, in the resulting image, $99.6 \%$ 
of the pixels contain unmodified MIPS intensities, $0.3 \%$ of the pixels contain scaled MSX intensities, and in between $0.1 \%$ of the pixels contain a weighted average of MIPS and scaled MSX intensities. (5) Lastly, we interpolate over $\sim 10$ small MIPS coverage gaps that occupy $0.03 \%$ of the image.

Despite the weighted averages, differences in the point-spread function (PSF) widths ( $\sim 18^{\prime \prime} .3$ for $M S X, \sim 6^{\prime \prime}$ for MIPS) create artifacts where $M S X$ data replace saturated MIPS point sources. Therefore, any quantitative analysis on MIPS saturated sources should be performed on the original MSX images, rather than the combined MIPS $+M S X$ images. The combined images are useful for qualitative evaluation of the structure and color of bright extended emission, and of course, for any unsaturated emission.

\subsection{MIPS Survey at $70 \mu \mathrm{m}$}

In order to obtain more complete SEDs of some of the high latitude $24 \mu \mathrm{m}$ sources that show excess emission at $4.5 \mu \mathrm{m}$, we selected a total of nine such sources distributed at high Galactic latitudes within our survey. These sources were observed separately with MIPS at $70 \mu \mathrm{m}$. The lowlatitude sources were not selected due their possible saturation at low latitudes in the plane. These targets were observed in MIPS photometry mode with the exposure time for each object of $3 \mathrm{~s}$ and default pixel scale of $10^{\prime \prime}$. All observations were obtained between 2007 September 21 and 23. These data were reduced using the MIPS Instrument Team's Data Analysis Tool version 3.06 (Gordon et al. 2007). Aperture photometry was performed utilizing a set of custom IDL scripts. The data were flux calibrated using the conversion factor of $702 \pm 35 \mathrm{MJy}$ $\mathrm{sr}^{-1}$ per raw MIPS units (5\% uncertainty) following Gordon et al. (2007). An aperture of $35^{\prime \prime}$ was used, with a sky radius of $39^{\prime \prime}-65^{\prime \prime}$. The aperture correction for this configuration is 1.185 .

\subsection{Radio Surveys}

Radio continuum observations at $\lambda 20 \mathrm{~cm}$ surveyed the $-2^{\circ}<$ $l<5^{\circ}$ and $|b|<40^{\prime}$ region with a spatial resolution of $\approx 30^{\prime \prime}$ and $10^{\prime \prime}$ using two different array configurations of the Very Large Array (VLA) of the National Radio Astronomy Observatory ${ }^{10}$ (Yusef-Zadeh et al. 2004). Another recent survey at $20 \mathrm{~cm}$ has extended this region to higher positive latitudes of up to $1^{\circ}$ (Law et al. 2008). We obtained 32 overlapping images at $20 \mathrm{~cm}$ from these two surveys and convolved individual images with a Gaussian having a FWHM $=12^{\prime \prime} .8 \times 12^{\prime \prime} .8$ before the images were mosaiced.

This region has also been observed at 20 and $6 \mathrm{~cm}$ with the VLA (Becker et al. 1994; Zoonematkermani et al. 1990). However, these surveys are short snapshot observations of a region dominated by bright sources with a wide range of angular scales and poor sensitivity and so are not useful for comparison with infrared sources due to the nonuniform $u v$ coverage. The detection of point sources at 90 and $20 \mathrm{~cm}$ (Nord et al. 2004; Yusef-Zadeh et al. 2004; Law et al. 2008) does suffer from the artifacts introduced by the lack of $u v$ coverage. More recently, C. C. Lang et al. (2009, in preparation) have completed a $6 \mathrm{~cm}$ survey of the Galactic center region with a uniform $u v$ coverage. We have used this data set to measure the fluxes of several point sources in the G359.43+0.02 cluster, as described below.

\footnotetext{
10 The National Radio Astronomy Observatory is a facility of the National Science Foundation, operated under a cooperative agreement by Associated Universities, Inc.
}

\subsection{Other Surveys}

A survey of Spitzer/IRAC observations of the central $2^{\circ} \times 1.4$ $(\sim 290 \times 210 \mathrm{pc})$ was recently conducted in the four wavelength bands at 3.6, 4.5, 5.8, and $8 \mu \mathrm{m}$ (Stolovy et al. 2006; Ramirez et al. 2008; Arendt et al. 2008). The catalog presented in Ramirez et al. (2008) includes point-source photometry from the IRAC survey and correlates those sources with previously published photometry from Two Micron All Sky Survey (2MASS) at $J, H$, and $K_{s}$ bands (Skrutskie et al. 1997).

A Submillimeter Common-User Bolometer Array (SCUBA) survey of the Galactic center covered a region of $2^{\circ} \times 0.5$ at 450 and $850 \mu \mathrm{m}$ with a spatial resolution of $8^{\prime \prime}$ and $15^{\prime \prime}$, respectively (Pierce-Price et al. 2000). Given the low resolution of the submillimeter data, the detected submillimeter flux is used as upper limits at $450 \mu \mathrm{m}$ and $850 \mu \mathrm{m}$ (Di Francesco et al. 2008) in the SED of individual point sources. However, large-scale submillimeter maps of extended sources at 450 and $850 \mu \mathrm{m}$ have been used for comparison with radio and mid-IR images.

\section{LARGE-SCALE DISTRIBUTION OF $24 \mu \mathrm{m}$ EMISSION}

\subsection{Extended Sources}

Figure 1(a) shows the large-scale view of the surveyed region at $24 \mu \mathrm{m}$. In order to bring out the weak sources, the sources distributed between $-1.8<l<0.8$ are burned out in this figure. The mean brightness of this region is roughly 4-5 times higher than the region beyond the inner few degrees of the Galactic center. Several extended H II regions are identified to have radio continuum and submillimeter counterparts (YusefZadeh et al. 2004; Pierce-Price et al. 2000). An enlarged view of the burned-out $3^{\circ} \times 2^{\circ}$ region is shown in Figure 1(b). Prominent Galactic center H II complexes along the Galactic plane are associated with Sgr A-E, the Arches and the Pistol, all of which are labeled, as shown in Figure 1(c). We note two extended $24 \mu \mathrm{m}$ emitting features G0.23-0.05 and G0.31-0.07 lying between the radio Arc and Sgr B1. The radio Arc, which includes the Arches cluster and the Pistol nebula, consists of a network of nonthermal radio filaments running perpendicular to the Galactic plane (Yusef-Zadeh et al. 1984; Lang et al. 1999). We note a number of parallel filamentary features at $24 \mu \mathrm{m}$ associated with G0.31-0.07 which will be discussed elsewhere. At positive latitudes, two extended clouds known as the western and eastern Galactic center lobes are shown prominently in Figure 1(b) near $l \sim-0.5$ and $\sim 0.2$, respectively. The striking structure of the western lobe with its strong $24 \mu \mathrm{m}$ emission coincides with AFGL 5376 (Uchida et al. 1990). In the eastern lobe, two columns of $24 \mu \mathrm{m}$ features with an extension of about $30^{\prime}$ run parallel to each other away from the Galactic plane. These features lie in the region of the Galactic center lobe that is known to emit strong synchrotron emission (Law et al. 2008). The "double-helix" nebula (Morris et al. 2006) lies along the northern extension of the eastern linear feature in the Galactic center lobe. The southern ends of these linear features appear to be pointed toward the large-scale ionized features associated with the Arches cluster G0.12+0.02 and a cluster of H II regions (H1-H5; Yusef-Zadeh \& Morris 1987; Zhao et al. 1993). In addition, there are several counterparts to foreground $\mathrm{H} \alpha$ emission line nebulae, RCW 137, 141, and 142, which are prominent at $|b|>0.2$.

At positive longitudes, a string of IRDCs is concentrated between $1 \sim 0.2$ and Sgr B2 near $l=0.7$. Submillimeter emission from these clouds is prominent at 450 and $850 \mu \mathrm{m}$ 
No. 1, 2009

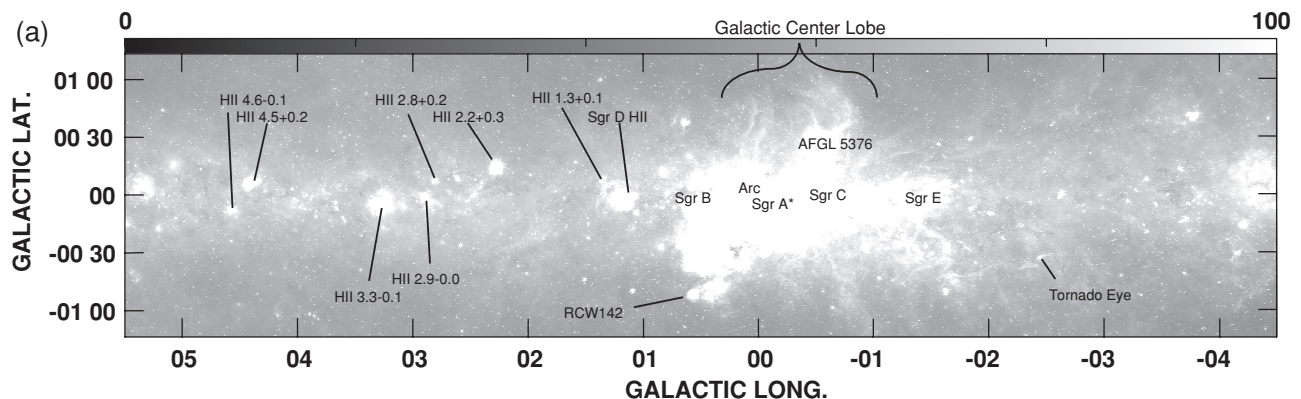

(b)
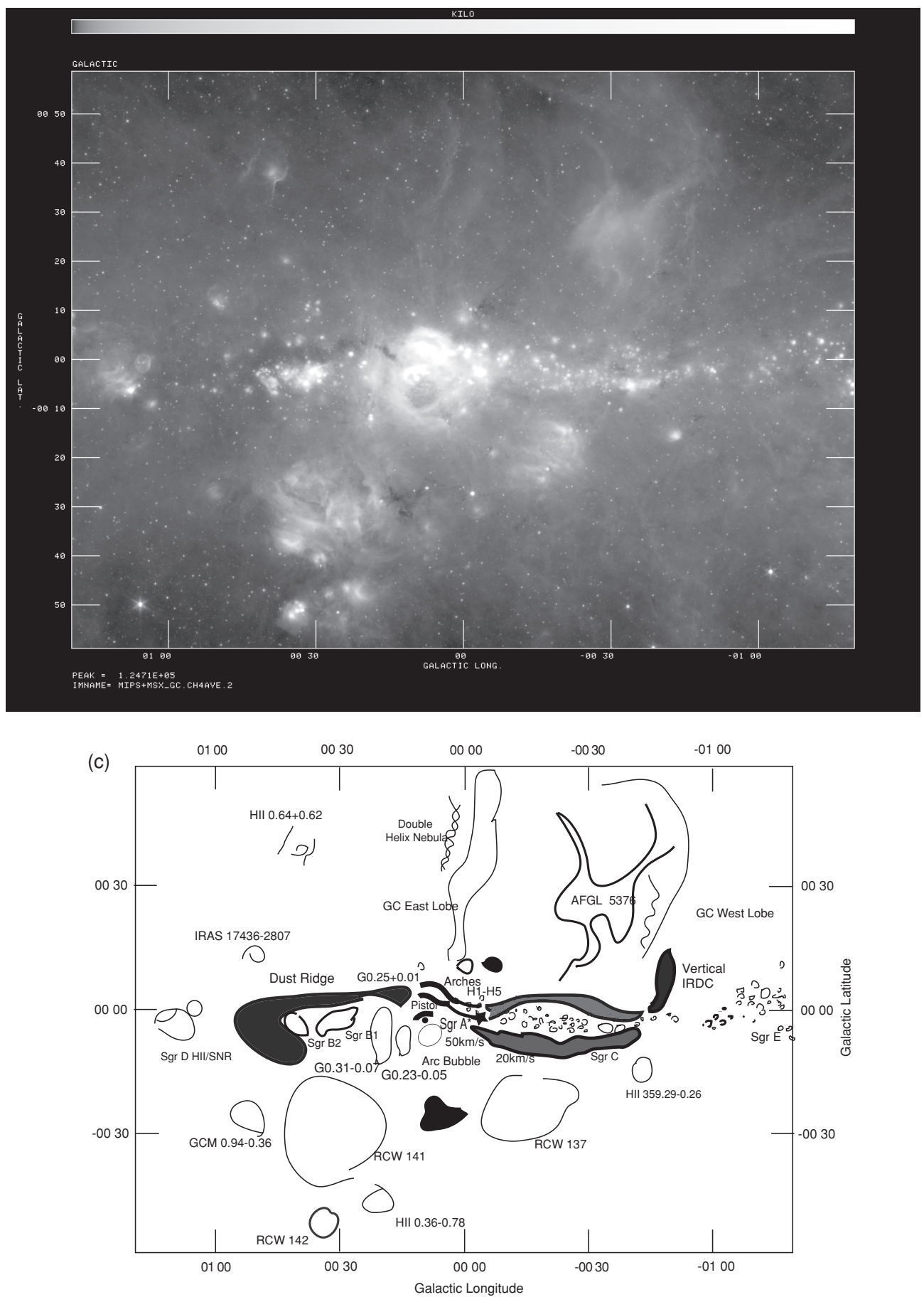

Figure 1. (a) $24 \mu \mathrm{m}$ image of the inner $\sim 10^{\circ} \times 2.5(l \times b)$ of the central region of the Galaxy based on combining MIPS and MSX data. (b) A close-up view of the central $2.5 \times 2^{\circ}$ showing the prominent H II complexes (Sgr A - E) at $24 \mu \mathrm{m}$. (c) A schematic diagram of prominent features detected at $24 \mu \mathrm{m}$ from the region shown in (b) 

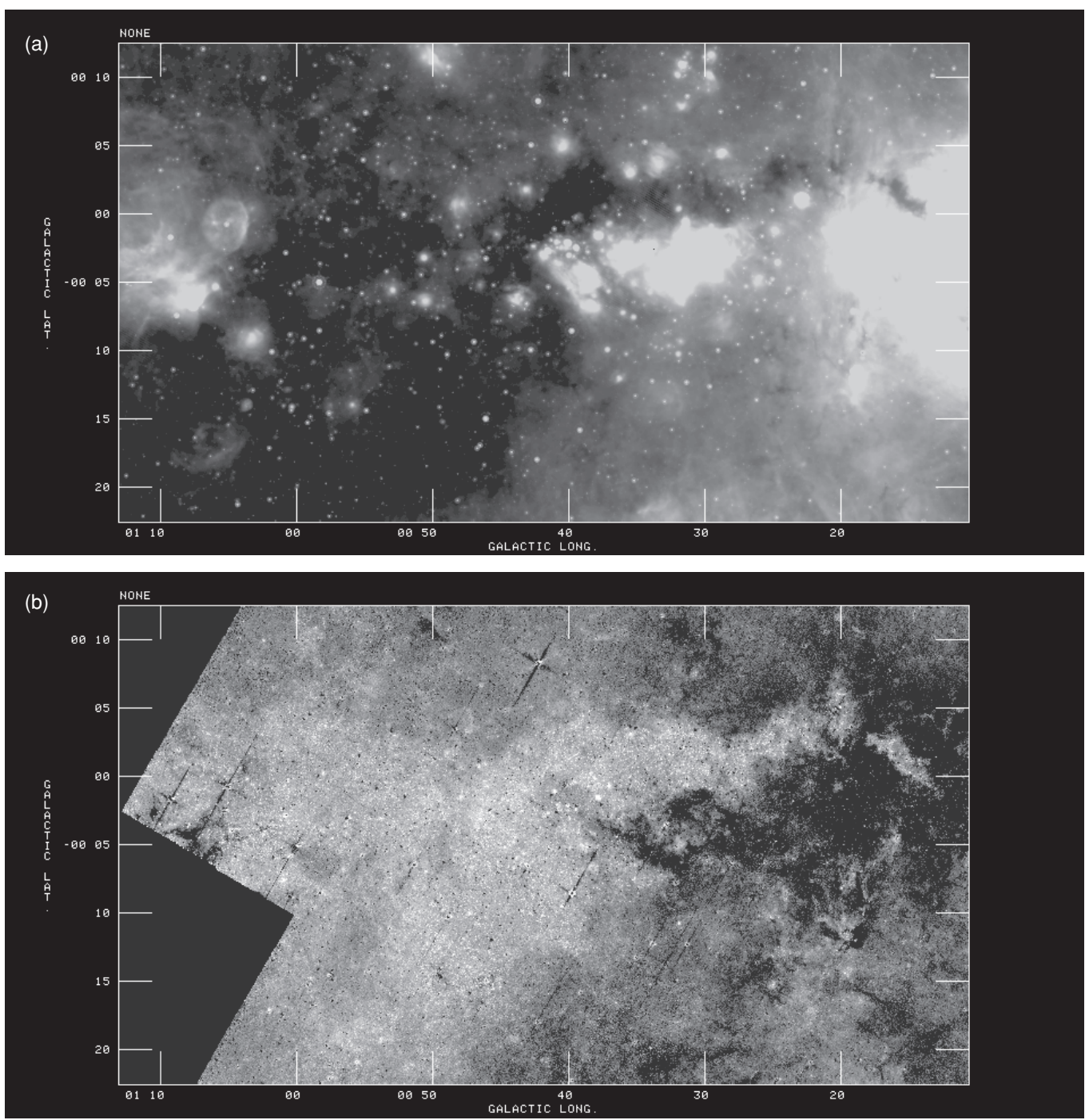

Figure 2. (a) $24 \mu \mathrm{m}$ emission from prominent chain of IRDCs on the positive longitude side of the Galactic center. The thickest cloud surrounds Sgr B2 to the left near $l=0.7$. (b) Identical to (a) except that the ratio of IRAC images is displayed. Positive values of this ratio are shown in light color which reveals low contrast features of the IRDCs. The ratio image is constructed by $I(4.5) /\left[I(3.6)^{1.4} \times I(5.8)\right]^{0.5}$.

(Lis \& Carlstrom 1994; Pierce-Price et al. 2000). Figure 2(a) displays an enlarged view of $24 \mu \mathrm{m}$ emission from dust clouds and stellar sources distributed at positive longitudes. With careful adjustment of the overall background level, the ratio of IRAC images, such as $I(4.5) /\left[I(3.6)^{1.4} \times I(5.8)\right]^{0.5}$, are used to highlight the location and structure of the IRDCs. This ratio is high for the IRDCs (Figure 2(b)). The bright feature in this figure corresponds to a dust lane that consists of a string of dust clouds. These clouds appear narrow near $l=0.25$ and become increasingly wider extending toward negative latitudes near Sgr B2. The kinematics of this dust lane have been studied in numerous molecular line surveys of this region (Oka et al. 1998; Martin et al. 2004).

At negative latitudes, the distribution of dust emission at $24 \mu \mathrm{m}$ and the ratio map are shown in Figures 3(a) and (b), respectively. IRDCs are detected toward the well known molecular clouds M-0.13-0.08 (20 $\left.\mathrm{km} \mathrm{s}^{-1}\right)$ and M-0.02-0.07 (50 km s' ${ }^{-1}$ Herrnstein \& Ho 2005; Armstrong \& Barrett 1985). Both clouds are located near the Galactic center. One of the largest structures in the ratio map is the pair of two dust lanes that run parallel to each other along the Galactic plane between $l=0^{\circ}$ and $l=-40^{\prime}$ on the positive and negative latitudes. The previous CS (1-0) line study of this region has shown the counterpart to the positive latitude dust lane to have a coherent kinematic structure. We also note an elongated dust cloud (called "Vertical IRDC" in Figure 1(c)) running toward positive latitudes at $l=-40^{\prime}, b=4^{\prime}$ with a latitude extent of $12^{\prime}$. The prominence of this feature with respect to the dust lanes in the ratio map suggests that it is a foreground cloud.

\subsection{Color Distribution of Extended Emission}

In order to analyze the color distribution at different bands, a close-up view of the central region of the $24 \mu \mathrm{m}$ survey is cropped to the exact size of the $8 \mu \mathrm{m}$ survey (Stolovy et al. 2006). Figure 4 (a) shows the combined 24,8 , and $4.5 \mu$ m images with red, green, and blue colors, respectively. Figure 4(b) shows only the $3.6,4.5$, and $8 \mu \mathrm{m}$ images in blue, green, and red colors, respectively. Overall, there is a great deal of nonuniformity in the color distribution detected between IRAC and MIPS images, as shown in Figure 4(a). In contrast, uniform color distribution is noted in IRAC images, as shown in Figure 4(b) (Arendt 

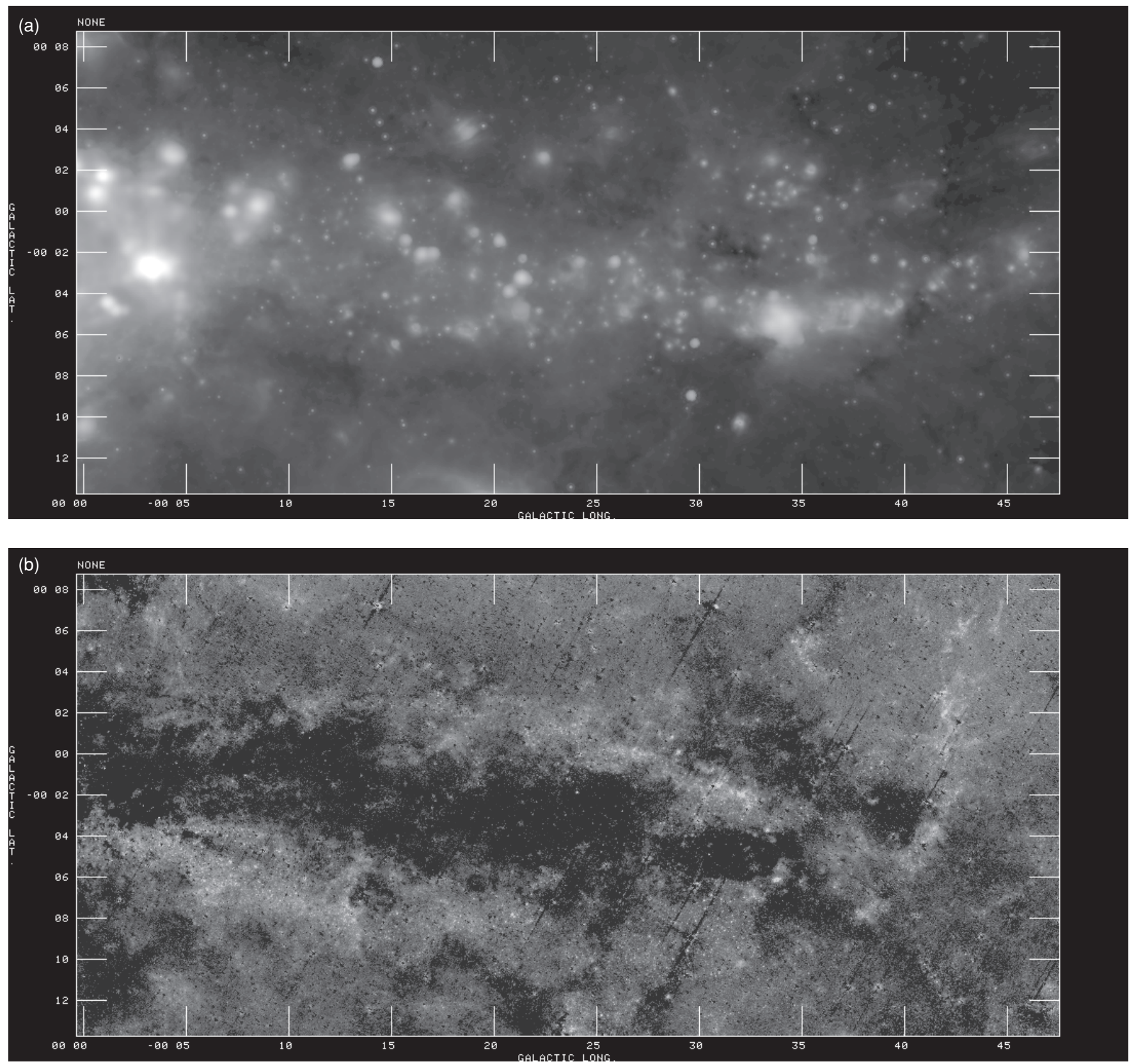

Figure 3. (a) Same as Figure 2(a) except that $24 \mu \mathrm{m}$ distribution from the negative longitude side is selected. Note the distribution of stellar objects along the Galactic plane. (b) As in Figure 2(b) regions with higher intensity ratios reveal details in the structure of IRDCs. Most of the stellar sources seen in (a) are mainly distributed between the two extended IRDCs running parallel to the Galactic plane.

et al. 2008). One of the main reasons for the nonuniformity of colors is that $24 \mu \mathrm{m}$ emission is an excellent diagnostic of local sources of heating of large dust grains. This is in part due to the fact that thermal emission from large dust grains at $24 \mu \mathrm{m}$ becomes significantly enhanced when the radiation field is only 100 times that of the local interstellar radiation field (ISRF) in the solar vicinity (Arendt et al. 2008). In contrast, $8 \mu \mathrm{m}$ emission mainly arises from aromatics (hereafter PAHs) that are excited by the ISRF and nearby OB stars. Thermal emission from small dust grains becomes strong enough to compete with the PAH emission in the $8 \mu \mathrm{m}$ band only when the local radiation field is about $10^{4}$ times that of the solar neighborhood (Li \& Draine 2001). Thus, there is much less variation in the $8-5.8 \mu \mathrm{m}$ color than in the $24-8 \mu \mathrm{m}$ color. These color distribution images based on IRAC and MIPS data place a lower limit of $\sim 100$ times the ISRF throughout the Galactic center region with the exception of the regions known to have a very strong radiation field such as the Arches and Sgr A (see Arendt et al. 2008).

Several extended sources show a distinct pattern of excess $24 \mu \mathrm{m}$ emission (red in Figure 4(a)) surrounded by $8 \mu \mathrm{m}$ emission (green and yellow in Figure 4(a)). The $24 \mu \mathrm{m}$ excess (or $8 \mu \mathrm{m}$ deficit) can be accounted for by the destruction of PAHs by local sources of UV radiation or shocks (Watson et al. 2008; Churchwell et al. 2007; Povich et al. 2007). In contrast to small grains and PAHs that can be destroyed, the large dust grains reradiate the absorbed UV radiation at long wavelengths, $24 \mu \mathrm{m}$. The most striking example that shows these effects is the optical $\mathrm{H} \alpha$ line emission nebulae RCW 137, 141 , and 142 which are foreground sources and are internally heated. PAHs are destroyed close to the source of heating, but are excited at the edge of the cloud by the external interstellar medium (ISM) radiation field. The $24 \mu \mathrm{m}$ diffuse emission 

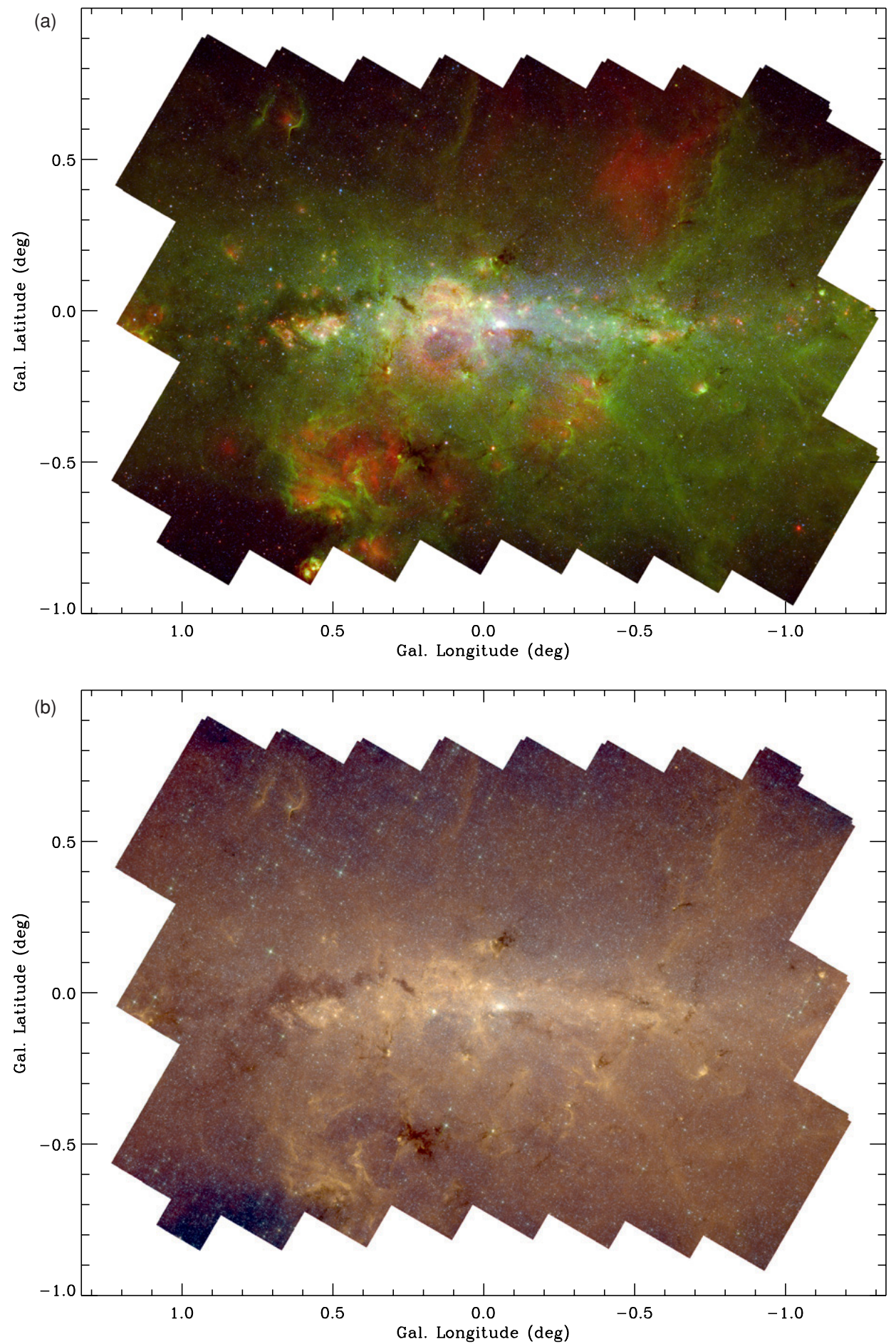

Figure 4. (a) Color images of the 24, 8, and $3.6 \mu \mathrm{m}$ emission from the central $2^{\circ} \times 1.4$ are shown in red, green, and blue colors, respectively. The diffuse clouds peaking at $24 \mu \mathrm{m}$ are centrally heated whereas the emission in green color is mainly due to PAHs in photodissociation regions. (b) Similar to (a) except that the 4.5, 5.8 , and $8 \mu \mathrm{m}$ images are shown in blue, green, and red colors, respectively.

(A color version of this figure is available in the online journal.)

along the Galactic plane traces local sources of heating by H II regions.

There is another type of $24 \mu \mathrm{m}$ source that shows neither $8 \mu \mathrm{m}$ counterpart nor any evidence for embedded sources of heating. AFGL 5376 (Uchida et al. 1990) is one example that is located at high latitudes. G0.85-0.44 is another source that shows similar characteristics. Alternative mechanisms must be responsible for heating these clouds. One candidate is the high flux of cosmic rays that could be responsible for heating large dust grains but not PAHs.

\subsection{Infrared Dark Clouds}

We note several IRDCs in Figure 4(a) with different levels of darkness. These cold, dark clouds are seen in absorption against the diffuse background emission. The IRDCs are optically thick even at $24 \mu \mathrm{m}$. Therefore, their apparent surface brightnesses 
are more of an indication of the emitting column density on the line of sight to the cloud (i.e., the cloud's distance) than a sign of the optical depth of the IRDCs. For example, we note that the IRDCs at high and low Galactic plane latitudes, such as G0.2+0.47 and G0.67+0.18 are the darkest clouds, suggesting that they are distributed locally along the line of sight.

As was shown in the ratio maps, the large-scale distribution of IRDCs forming a dust lane implies an early phase of massive star or star cluster formation in the Galactic center region. IRDCs are dense $\left(>10^{5} \mathrm{~cm}^{-3}\right)$, cold $(<25 \mathrm{~K})$ and attain high column densities $\left(10^{23} \mathrm{~cm}^{-2}\right)$ in the Galactic disk (Egan et al. 1998; Carey et al. 2000; Simon et al. 2006). The extreme physical properties of the clouds in the Galactic center region should be even more suitable for young, massive star cluster formation. We note the string of IRDCs are coincident with the "Dust Ridge" (Lis et al. 2001), as shown in Figure 1(c). What is remarkable is a large-scale east-west ridge of IRDCs tracing dense molecular gas between G0.25+0.01 and Sgr B2. This continuous eastwest IRDC ridge shows a sinusoidal appearance on a scale of $\sim 5^{\prime}$ corresponding to $12 \mathrm{pc}$. The western half of this largescale ridge of IRDCs is quiescent (Lis et al. 2001) in its star formation activity whereas the eastern half shows spectacular on-going star formation. The string of submillimeter emitting molecular clouds includes G0.25+0.01 and the Sgr B complex and is darker than the well known 20 and $50 \mathrm{~km} \mathrm{~s}^{-1}$ molecular clouds M-0.13-0.08 and M-0.02-0.07 as well as clouds in the negative longitude side of the Galactic center. This behavior suggests that the positive-longitude IRDCs are closer than the 20 and $50 \mathrm{~km} \mathrm{~s}^{-1}$ and Sgr C clouds. The large-scale distribution of IRDCs traces coherent molecular lanes running parallel to the nuclear disk (Bania 1977; Bally et al. 1988; Oka et al. 2005; Tsuboi et al. 1999; Martin et al. 2004), as shown in the ratio maps of Figures 2 and 3 . The color contrast of the coherent, largescale dust lane suggests that the molecular gas in the central molecular zone is oriented in the same direction as that of the well known stellar bar (Binney et al. 1991; Stark et al. 1991).

\subsection{Distribution of Compact Sources}

The blue color (4.5 $\mu \mathrm{m}$ emission) in Figure 4(a) represents the stellar density distribution in Sgr A where the evolved stellar core of the bulge peaks. This is mostly unresolved stellar sources, not intrinsically diffuse emission. A number of red $(24 \mu \mathrm{m})$ point sources are also seen in this figure that appear to be embedded within IRDCs suggesting that they are protostellar candidates, as will be discussed in Section 5. The red color reveals embedded stellar sources that heat surrounding dust grains to high temperatures. Numerous compact H II regions with radio continuum counterparts are distributed along the Galactic plane near the Galactic center.

To reveal the distribution of intrinsically red compact sources against the strong extended emission, we constructed a $24 \mu \mathrm{m}$ to $8 \mu \mathrm{m}$ intensity ratio image, which is presented in Figure 5(a). Regions with relatively strong $24 \mu \mathrm{m}$ emission are light whereas regions with strong $8 \mu \mathrm{m}(\mathrm{PAH})$ emission are dark. Most of the emission from the ISM (including that on the line of sight to IRDCs) disappears into a flat gray background. The disappearance of IRDCs is because they are opaque at both $8 \mu \mathrm{m}$ and $24 \mu \mathrm{m}$. The extinction ratio $\left(A_{8 \mu \mathrm{m}} / A_{24 \mu \mathrm{m}} \sim 1 ; \mathrm{Li}\right.$ $\&$ Draine 2001) implies a lack of reddening even for clouds that are not opaque (e.g., their edges). The "removal" of the typical ISM emission and dark clouds accentuates a number of structures and helps reveal some fainter, compact, and extended sources with excess $24 \mu \mathrm{m}$ emission. The most striking feature in the ratio map is the distribution of compact emission from a high concentration of strong IR-excess objects, candidate YSOs or UC HII regions distributed between $l=0^{\circ}$ and $l=-1.2$.

Figure 5(b) shows the relationship between the compact $24 \mu \mathrm{m}$ point sources and the molecular gas. It is a composite image indicating $24 \mu \mathrm{m}$ in green and $450 \mu \mathrm{m}$ in red. Submillimeter emission at $450 \mu \mathrm{m}$ is optically thin and is known to be an excellent tracer of molecular gas distributed in the central molecular zone. The comparison of the distribution of $24 \mu \mathrm{m}$ emission and the ratio map shows two features. One is the distribution of extended submillimeter emission being strongest in the positive longitude side of the Galactic center. It is clear that molecular gas distribution at $l>0^{\circ}$ is more uniform and more intense at $450 \mu \mathrm{m}$ than that at negative longitudes. Similar asymmetric structure is also noted in CS and CO line observations (Bally et al. 1988; Martin et al. 2004; Oka et al. 2005; Tsuboi et al. 1999). This asymmetric distribution shows $2 / 3$ of the mass of molecular gas on the positive longitude side of the Galactic center region (Bally et al. 1988; Oka et al. 2005; Martin et al. 2004). The presence of such an asymmetry is mainly due to massive clouds associated with $\mathrm{Sgr} \mathrm{B} 2$ and the dust ridge and a lack of massive, dense clouds at $l<0^{\circ}$ with the exception of Sgr C.

The second feature is the distribution of $450 \mu \mathrm{m}$ emission at negative longitudes showing that $24 \mu \mathrm{m}$ sources anticorrelate with the distribution of dust clouds. Remarkably, the distribution of $24 \mu \mathrm{m}$ point sources appears to be sandwiched by two layers of dust and gas clouds running parallel to the Galactic plane and symmetrically distributed with respect to the Galactic center. The "bow-tie" dust layers, as schematically drawn in Figure 1(c), stretch along both positive and negative longitudes and coincide with IRDCs at $8 \mu \mathrm{m}$ and $24 \mu \mathrm{m}$. Submillimeter emission is also noted from a cloud at negative latitudes $b=-5^{\prime}$. This feature is the negative latitude counterpart to the layer of submillimeter emitting cloud in the region between G0.25+0.01 and Sgr B1 at positive longitudes. The negative latitude submillimeter layer does not have an IRDC counterpart, implying that it is most likely located on the far side of Sgr B2. Thus, it is likely that these molecular layers are closer to us at positive longitudes than at negative ones. What is remarkable from the mid-IR and submillimeter images is that the "bow-tie" structure in fact consists of two layers of molecular and dust clouds that are symmetrically distributed with respect to the Galactic center. However, it is most likely that the positive longitude side of these molecular layers is closer to us than those at negative latitudes.

We also note that unlike the gas and dust distributions, the $24 \mu \mathrm{m}$ point sources are more uniformly distributed on the negative longitudes than at positive ones. Given that the chain of IRDCs that include Sgr B2 are thought to be closer than those at negative longitude, it could be that they hide a distant population of $24 \mu \mathrm{m}$ sources and create an apparent asymmetry, as discussed in more detail below.

\section{CANDIDATE YSOs}

To investigate the nature of the $24 \mu \mathrm{m}$ compact sources, 8-24 $\mu \mathrm{m}([8]-[24] \mu \mathrm{m})$ CMDs are constructed for sources within $|b|=10^{\prime}$ and $|l|=1.4$, corresponding to the central $0.4 \times 0.05 \mathrm{kpc}$ region. The CMD of these sources identified in the positive and negative longitudes is shown in Figure 6. These data used the MIPS catalog at $24 \mu \mathrm{m}$ (Hinz et al. 2009) and IRAC catalog at $8 \mu \mathrm{m}$ (Ramirez et al. 2008). A total of 172,670 sources are detected, 70,794 and 101,876 of which are 


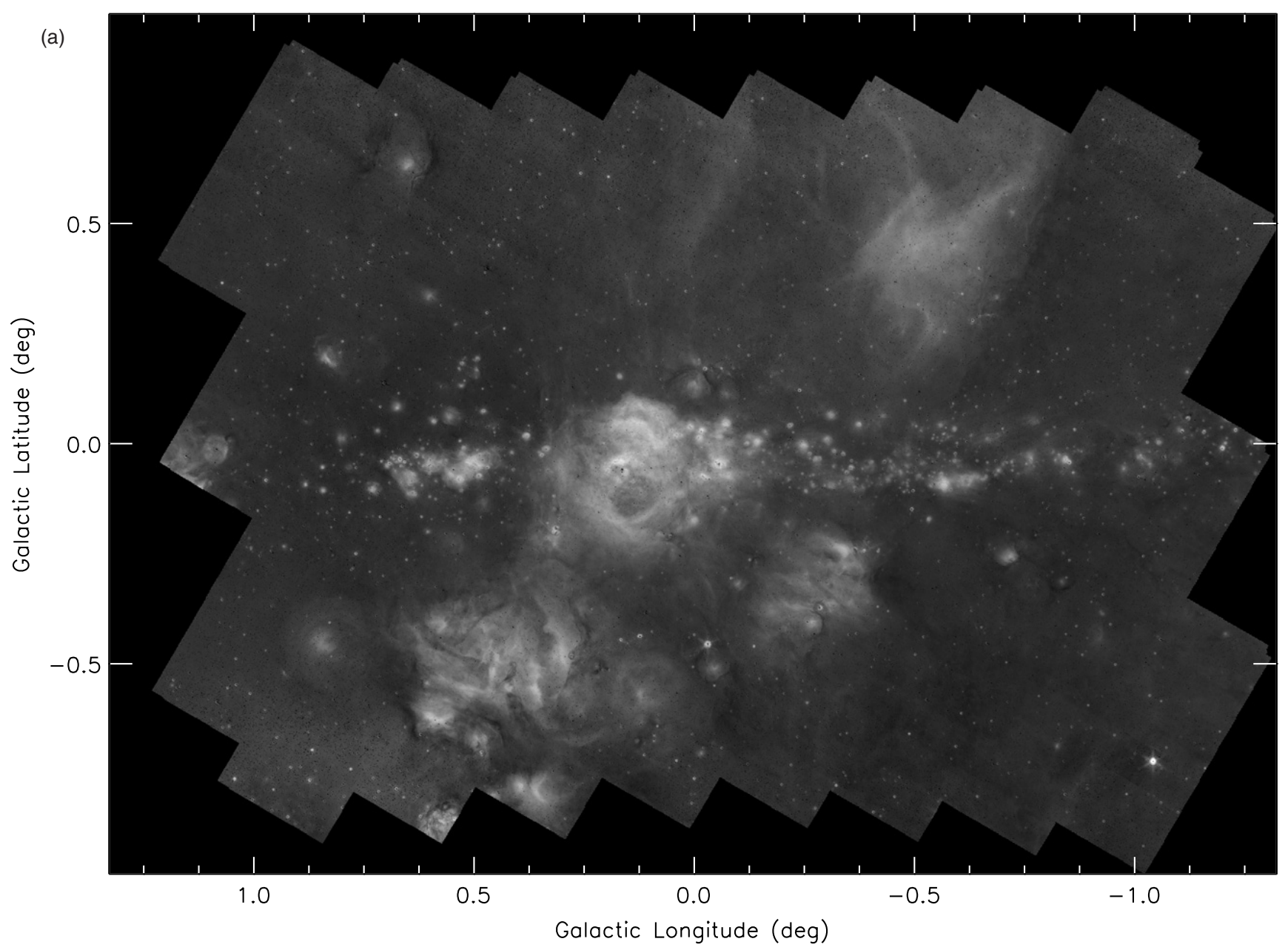

(b)

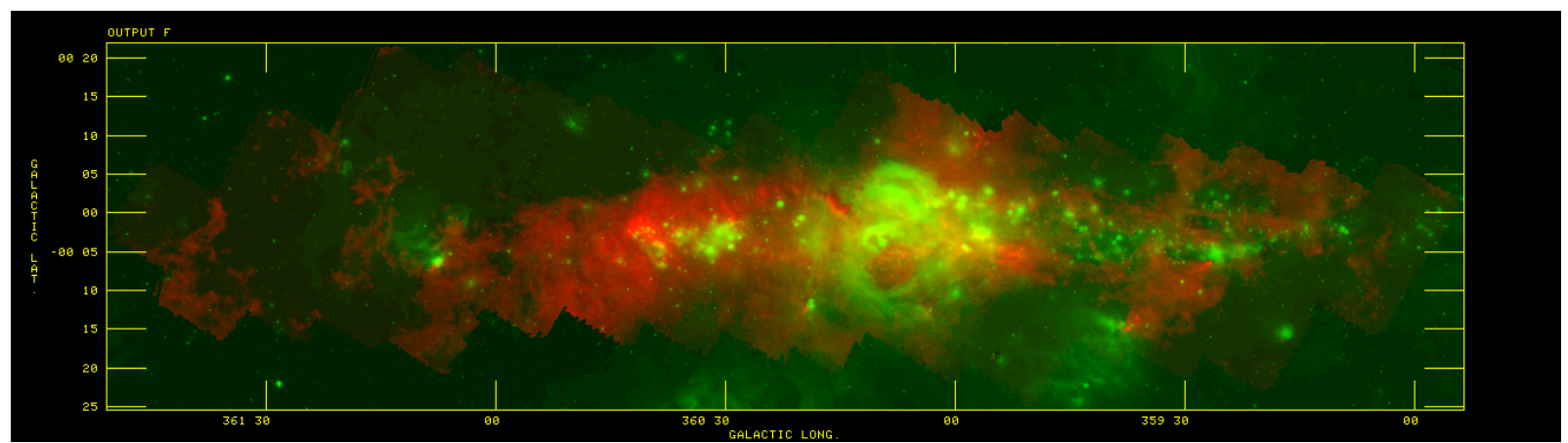

Figure 5. (a) Large-scale gray-scale ratio image $I(24 \mu \mathrm{m}) / I(8 \mu \mathrm{m})$ shows the bright region to be mainly due to $24 \mu \mathrm{m}$ emission whereas dark region shows the region dominated by $8 \mu \mathrm{m}$ emission. Note that the dark regions in this ratio image do not represent the IRDCs. (b) The distribution of $24 \mu \mathrm{m}$ emission in green color is shown against the distribution of $450 \mu \mathrm{m}$ emission (Pierce-Price et al. 2000) in red color.

(A color version of this figure is available in the online journal.)

found in the region restricted to positive and negative longitudes, respectively. There is an excess of 31,082 sources identified in negative longitudes. This asymmetry could be explained by the high extinction experienced by mid-IR sources in the region where dense molecular clouds are highly concentrated. Alternatively, the distribution of compact dusty sources could be due to an intrinsic excess of YSOs or evolved AGB stars. The dashed line in Figure 6 is chosen empirically, as discussed below, to separate the reddest YSOs from AGBs. Each of the two possibilities is discussed below.

$\mathrm{OH} / \mathrm{IR}$ stars are oxygen-rich, mass-losing cool giants that represent evolved AGB stars at the end of their lives (Habing
1996). These post-AGB stars are known to be dusty with excess emission at infrared wavelengths. YSOs are also known to have excess emission in infrared wavelengths and are characterized by dusty envelopes and disks that absorb the radiation from the central protostar (Whitney et al. 2003a; Adams et al. 1987). To explore the color of evolved stars in the Galactic center, we examined the spatial distribution of known $\mathrm{OH} / \mathrm{IR}$ stars in the region shown in Figure 6 using the $\mathrm{OH} 1612 \mathrm{MHz}$ catalogs (Lindqvist et al. 1992; Sevenster et al. 1997a, 1997b; Sjouwerman et al. 1998). OH/IR surveys are not affected by visual extinction. These surveys have uniformly sampled the region covered by IRAC observations. The shallow but 

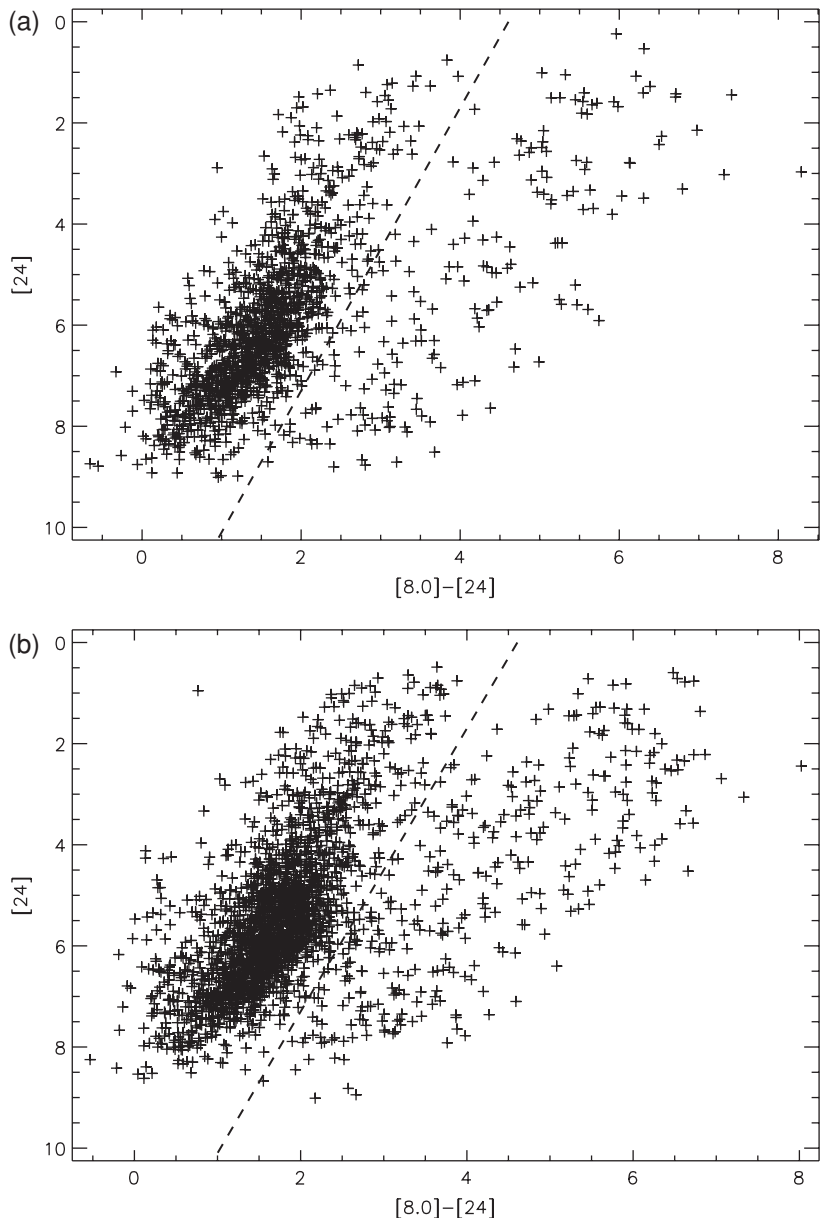

Figure 6. (a) CMD of $24 \mu \mathrm{m}$ sources covers the region $\mathrm{b} \pm 10^{\prime}$ and $0<1<1$. 4 . The dashed line is an empirical separator between evolved AGB stars (to the left) and candidate YSOs to the right. (b) Similar to (a) except that the search area includes $-1.4<1<0$

uniformly sampled survey by Sevenster et al. (1997a) finds a total of seven and nine $\mathrm{OH} / \mathrm{IR}$ stars in the restricted region, for positive and negative longitudes, respectively. Similarly, the spatial distribution of $\mathrm{OH} / \mathrm{IR}$ stars on a large scale is symmetric with respect to the Galactic center. Although, there are not many $\mathrm{OH} / \mathrm{IR}$ masers that we found to have infrared counterparts in the restricted region of our survey, we find no evidence of asymmetry in the distribution of $\mathrm{OH} / \mathrm{IR}$ stars with respect to the Galactic center.

To examine whether the observed color of the infrared sources (Ortiz et al. 2002; Ojha et al. 2007) can be due to the excess of YSOs, we first identify the color of OH/IR stars. The reddening toward these Galactic center sources is minimally affected by the differential extinction between 8 and $24 \mu \mathrm{m}$. Flaherty et al. (2007) have recently derived $A(8 \mu \mathrm{m})-A(24 \mu \mathrm{m})$ $\sim 0.0 \pm 0.05 \times A(\mathrm{~K})$. The color variation between 8 and $24 \mu \mathrm{m}$ should be $<0.2$ mag assuming $A(\mathrm{~K}) \sim 3.2$ or $\sim 30$ mag of visual extinction. Thus, the observed color of the sources is intrinsic. Here, we make an empirical assumption that the very red sources are likely to be candidate YSOs. Figure 7(a) shows the CMD of all the $24 \mu \mathrm{m}$ sources found in the surveyed region by IRAC. The colors of OH/IR stars that are found using the survey by Sevenster et al. (1997a) is shown as triangles in Figure 7(a) and are distributed to the left of the dashed line that was selected empirically. The color of $\mathrm{OH} / \mathrm{IR}$ masers is clearly distinguished from the reddest and faintest sources (by a few magnitudes). The dashed line in Figure 7(a) is the slope
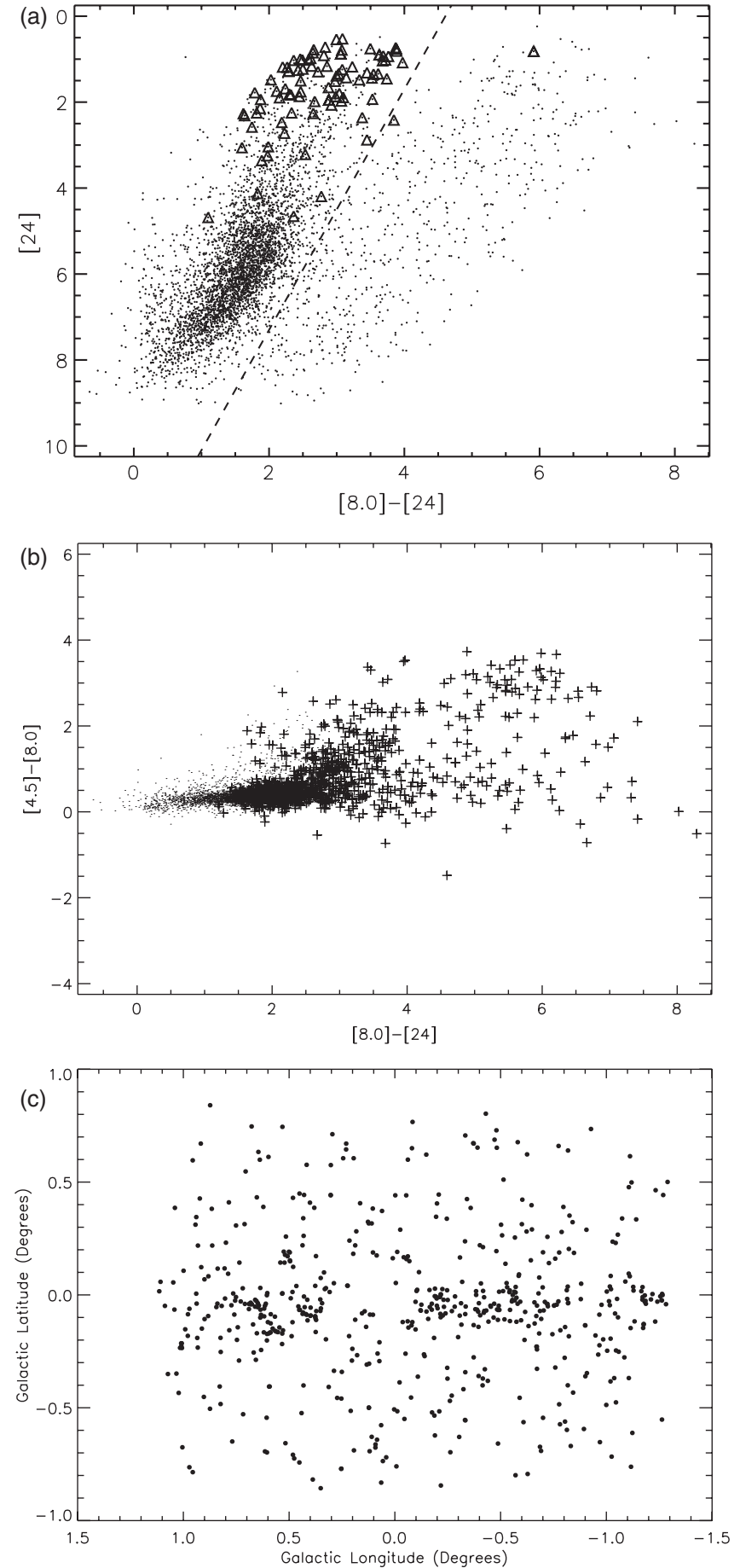

Figure 7. (a) CMD of the region covering $-10^{\prime}<b<10^{\prime}$ and $-1.4<l<1$. 4 . The triangles show the locations of known $\mathrm{OH} / \mathrm{IR}$ stars within the region. The dashed line is drawn to separate the $\mathrm{OH} / \mathrm{IR}$ stars from candidate YSOs with redder colors. A total of 4,541 sources is plotted as dots in the CMD. (b) Color-color-magnitude diagram of the region covered in (a). The crosses show the location of candidate YSOs identified in (a). (c) The spatial distribution of $24 \mu \mathrm{m}$ sources. The central hole centered on Sgr A is due to MIPS saturation at this wavelength.

that separates most of the YSO candidates to the right from the rest of the sources that have 24 and $8 \mu \mathrm{m}$ counterparts. The left side of the slope in Figure 7(a) indicates a trend of IR excess sources with luminosity. The CMD of the Large Magellanic Cloud (LMC) shows a similar trend which is due to a linear relationship between more luminous AGB stars with increasing radiation pressure, and more mass loss of the dust, and therefore 
more IR excess (see Figure 3(a) of Whitney et al. 2008). Thus, we believe the sources to the right of the slope in Figure 7(a) are dominated by candidate YSOs. The color-color diagram between [8] $-[24] \mu \mathrm{m}$ is also shown in Figure 7(b) where the candidate YSOs are shown as crosses. In the restricted $l= \pm 1.4$, $b= \pm 10^{\prime}$ region the total number of YSO candidates is found to be 347 and 212 distributed on the negative and positive longitudes, respectively. The spatial distribution of candidate YSOs with [8] - [24] greater than 4 is shown in Figure 7(c) revealing that the reddest infrared sources are distributed mostly along the Galactic plane. The numbers of YSO candidates are lower limits because the colors of stars in the saturated regions near $l \sim 0^{\circ}$ and $l \sim 0.2$ could not be examined (see Figure 7(c)). In spite of these restrictions, we note that most of the candidate YSOs are distributed in the negative latitudes, very close to the Galactic equator.

The saturated sources at $24 \mu \mathrm{m}$ as well as the contaminating sources limit our ability to address the issue of completeness. However, we believe that the distribution of OH/IR sources validates our empirical choice for the slope of the dashed line used to identify most of the YSO candidates. An additional step is taken in Section 5.3 to identify the fraction of YSO candidates via SED fitting of the sources selected from the right region of the CMD of Figure 7. As discussed below, $~ 60 \%-$ $65 \%$ of the total number of sources from the CMDs are SED fitted. Ultimately, future spectroscopic measurements of YSO candidates should be able to address the completeness issue.

\subsection{Excess Candidate YSOs in $l<0^{\circ}$ ?}

To test the possibility that there is an asymmetric distribution of candidate YSOs with respect to the Galactic center, we examined two tracers that are not affected by visual extinction. First, we examined compact radio continuum sources (YusefZadeh et al. 2004) and found 44 and 58 sources in the positive and negative longitude sides of the Galactic center, restricted to the same regions where candidate YSOs are found. It is known that Sgr B2 is one of the most active star-forming sites in the Galaxy at $l \sim 0.7$ (Mehringer et al. 1998; De Pree et al. 2005). We note that 16 of the 44 compact radio sources at the positive longitudes arise only from ultracompact $\mathrm{H}$ II regions in $\mathrm{Sgr} \mathrm{B} 2$. On the other hand, compact radio continuum sources in the $l<0^{\circ}$ region are distributed more uniformly than in the region at $l>0^{\circ}$. The excess number of 14 sources at negative longitudes combined with nonuniform distribution of compact radio continuum sources at positive longitudes imply an asymmetry of radio continuum sources that cannot be explained by excess extinction at positive latitudes. This is consistent with the excess of infrared sources in the negative longitudes of the Galactic center. Additional support for this picture comes from $\mathrm{H}_{2} \mathrm{O}$ maser study of the same region showing a similar distribution to that of compact $20 \mathrm{~cm}$ radio continuum sources. This survey used the Infrared Astronomical Satellite (IRAS) Galactic center catalog (Taylor et al. 1993) to search for $\mathrm{H}_{2}$ masers. The largest concentration of star-forming water masers is found in Sgr B2 whereas the star-forming $\mathrm{H}_{2} \mathrm{O}$ masers are populated uniformly in the negative longitude sides of the Galactic center (Taylor et al. 1993). For example, the targeted survey by Taylor et al. which excluded the Sgr B2 region, detected a total of 13 and one star-forming $\mathrm{H}_{2} \mathrm{O}$ masers at the negative and positive longitudes, respectively. Although water maser survey observations have not uniformly sampled the Galactic center region, the available data are consistent with the picture that the largest concentration of quiescent molecular clouds such as the string of IRDCs distributed in $l>0^{\circ}$ do not have a high density of $24 \mu \mathrm{m}$ sources, $\mathrm{H}_{2} \mathrm{O}$ masers, and ultracompact radio continuum sources (Lis \& Carlstrom 1994).

Since most of the candidate YSOs are distributed asymmetrically on the negative longitude side of the Galactic center, we constructed another histogram of candidate YSOs for $l<0^{\circ}$. This histogram should be a better representation of the scale height of the uniformly distributed YSO candidates. Gaussian fits to the narrow and broad components give FWHM $5.17 \pm 0.2$ centered at $b=-2.4$ and FWHM $\sim 28.6 \pm 2^{\prime}$ centered at $b=-0^{\prime} 02$, respectively. The scale height of the YSO candidates in $1<0^{\circ}$ is $h \sim 6.3 \mathrm{pc}$ is similar to $h \sim 7 \mathrm{pc}$ when all the sources at both positive and negative longitudes are included.

\subsection{Distribution of $24 \mu \mathrm{m}$ Sources}

To examine the distribution of YSO candidates as a function of latitude, a histogram was made using the data from the central $2.6 \times 2^{\circ}(l \times b)$. Figure 8 (a) shows a narrow and a broad component. The broad and narrow components are fitted with two Gaussians. The broad component is centered near $b \sim 0^{\prime}$ with a FWHM $=64^{\prime} .9 \pm 2^{\prime}$ whereas the narrow component is displaced with respect to the Galactic plane at $b=-3^{\prime}$ with a FWHM 5'.6 \pm 0.2 . Figure 8(b) shows the distribution of the candidate YSOs when they are restricted to $|b|<10^{\prime}$. This distribution is consistent with the narrow latitude distribution of YSO candidates with an offset with respect to the Galactic equator near $b=-2 \cdot 4$. This value is likely to be close to the true latitude of the Galactic plane since Sgr $A^{*}$, the massive black hole at the Galactic center, lies at $b=-2$ '.8. Unlike the broad distribution that is likely due to foreground sources toward the Galactic center, the narrow distribution of the candidate YSOs suggests a uniform sample of sources located along the midplane of the Galactic center. The scale height of the candidate YSOs is estimated to be $h \sim 7 \mathrm{pc}$ assuming the distance to the Galactic center is $8.5 \mathrm{kpc}$. Molecular line observations of this region based on CS (1-0) transition also finds a narrow scale height $h \sim 10$ pc (Bally et al. 1988). The narrow scale height of both candidate YSOs and molecular clouds can be used as a support for the association of infrared sources and the disk population of molecular gas distributed in the Galactic center region. To test that the reddest sources belong to a population of YSOs distributed in the midplane of the Galactic center region, the YSO candidates from CMDs of Figures 6 and 7 are presented in two-dimensional histograms of Figures 8(c) and (d) to emphasize the higher fraction of brighter, redder YSO candidates within $|b|<10^{\prime}$ and the exterior region $|b|>10^{\prime}$, respectively. These CMDs are selected from the point-source catalogs. The fraction of redder YSO candidates with [8] $-[24]>3$, relative to the total number of sources in the inner $10^{\prime}$ is found to be higher by about a factor of 5-10 higher than the region beyond the inner $10^{\prime}$. This suggests that there are more red YSOs in proportion to the total YSOs detected for the innermost region of the Galactic center than those found in the outermost region. There are 1720 YSO candidates, 599 of which are in the region restricted to $-1.4<l<1.4$ and $-10^{\prime}<b<10^{\prime}$; there are 347 and 252 sources distributed in the negative and positive longitudes, respectively.

To compare the scale heights of candidate YSOs and the bright $24 \mu \mathrm{m}$ point sources, we constructed Figure 9(a) which shows the distribution of bright unsaturated $24 \mu \mathrm{m}$ sources between 0 and $5 \mathrm{mag}$ as a function of latitude within $|l|<1.3$. The narrow and wide components reflect the scale height of all 

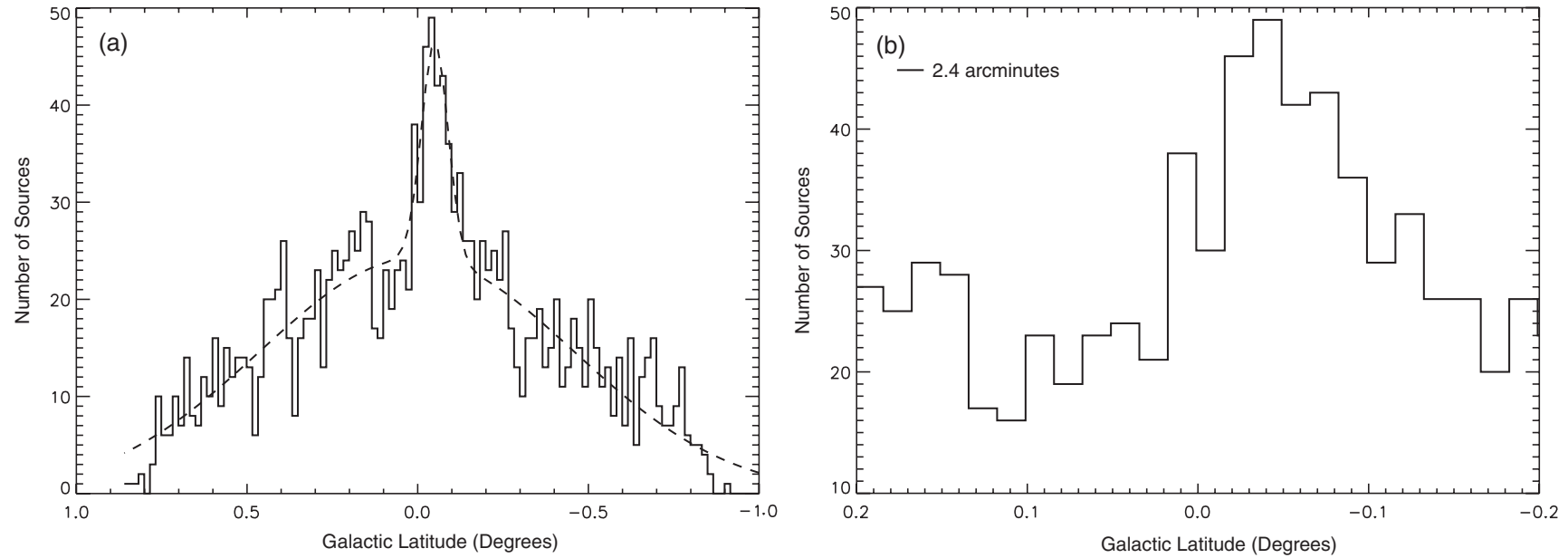

$|\mathrm{b}|<10^{\prime}$

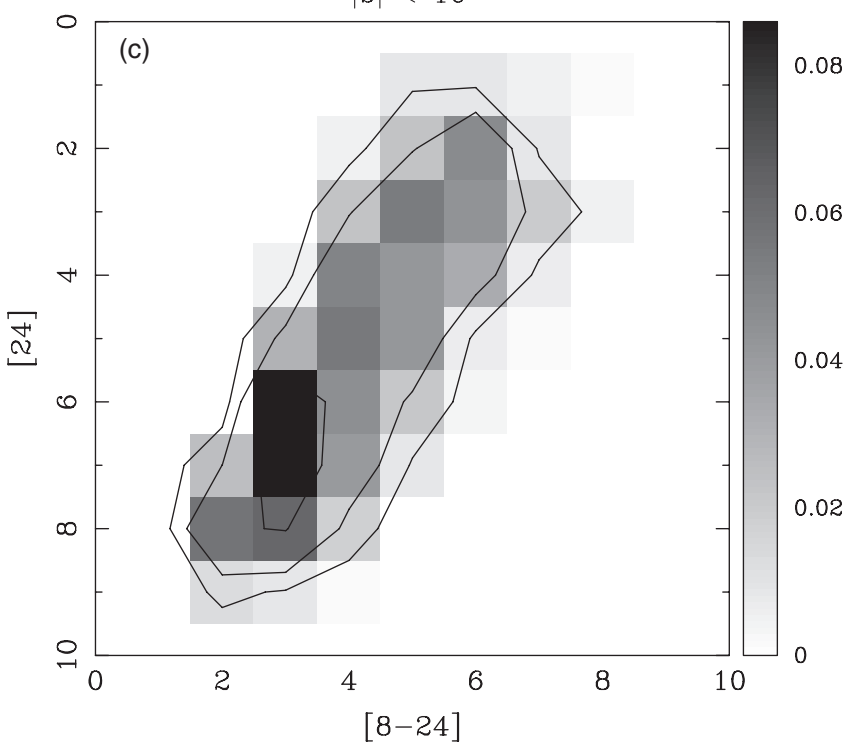

$|b|>10^{\prime}$

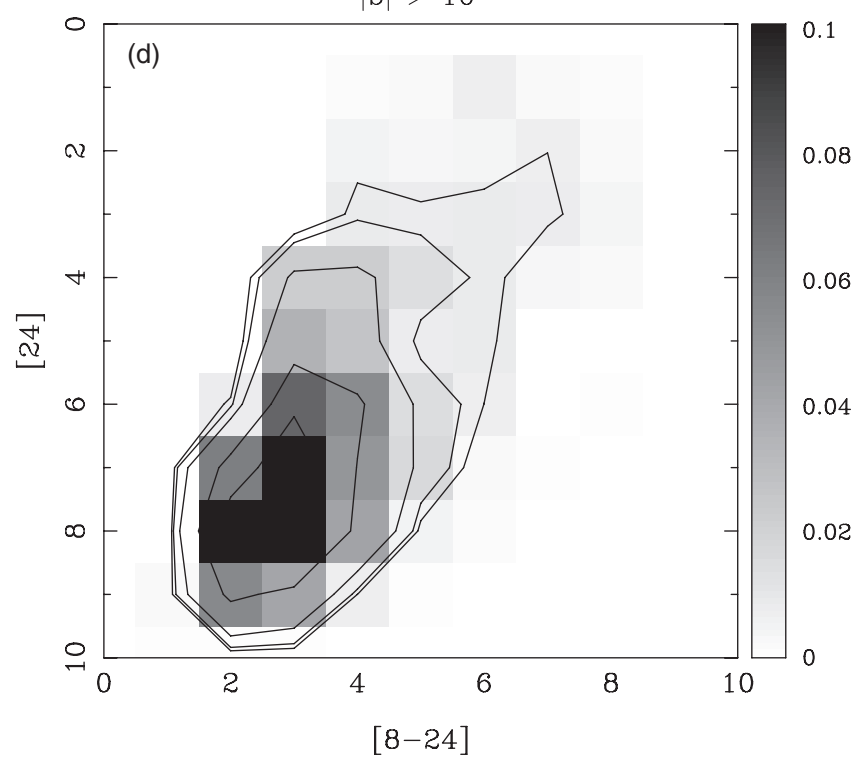

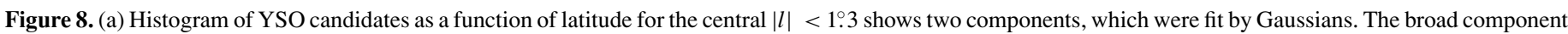

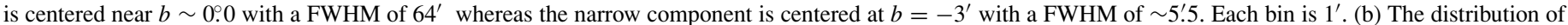

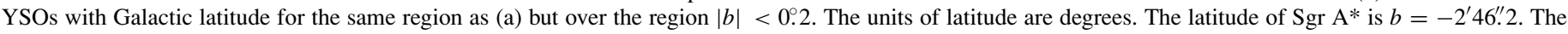

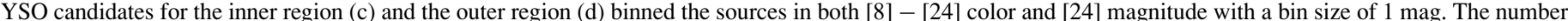

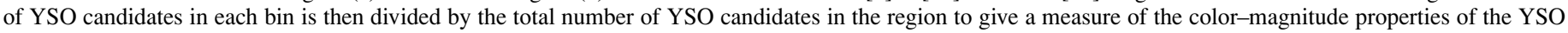

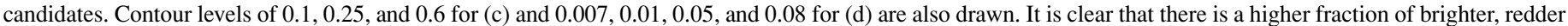
YSO candidates in the innermost region of the Galactic center when compared to outside sources.
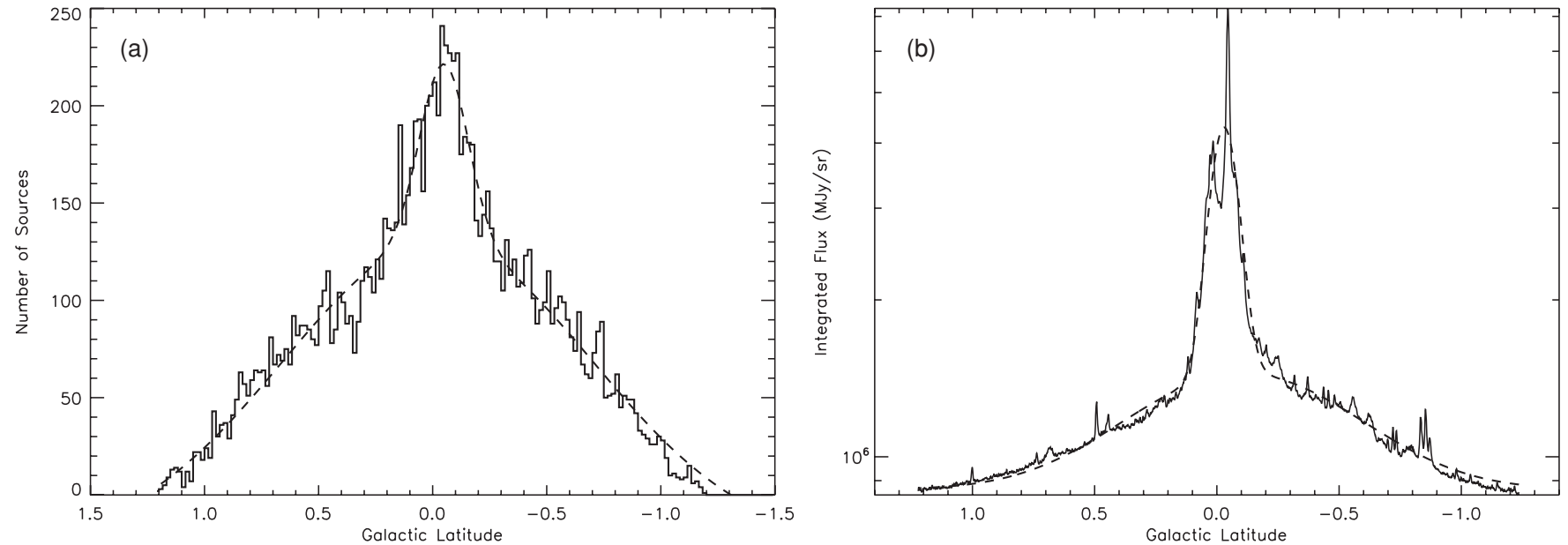

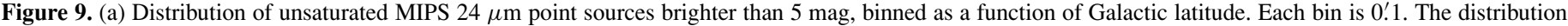

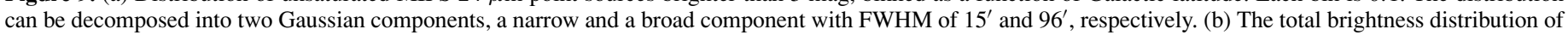
$24 \mu \mathrm{m}$ emission as a function of latitude based on the combined MIPS and MSX image using $|l|<4^{\circ}$. The units of latitude are degrees. 
bright stars which include both the evolved dusty stars and YSO candidates. The fitted values give two components with FWM $\sim 15^{\prime}$ and $96^{\prime}$, both of which are broader than those of the candidate YSOs. To measure the scale height of the bright dusty stars without being contaminated by the YSO candidates, we subtracted the candidate YSOs and determined the latitude distribution of dusty sources. Two components were fitted with FWHM $\sim 16^{\prime} .2 \pm 0.3$ and $105^{\prime} \pm 3^{\prime}$. The scale height of the narrow and bright component corresponds to a value of $\sim 20 \mathrm{pc}$. The higher values of the scale heights of bright dusty sources compared to YSO candidates suggest they are more evolved dynamically than the YSOs. An important implication of these scale heights is the support for a new population of YSOs distributed in the Galactic center region.

Using the mosaic image based on the combined MSX and MIPS data, we also constructed the brightness distribution of $24 \mu \mathrm{m}$ emission as a function of latitude and longitude. Figure 9(b) shows the latitude distribution where two broad and narrow components are detected. The broad background emission is subtracted followed by a Gaussian fit to the narrow component, giving a FWHM $\sim 8^{\prime}$ which corresponds to a scale height of $\sim 10 \mathrm{pc}$. This value is slightly thicker than the scale height of candidate YSOs having $h=7 \mathrm{pc}$ but close to the scale height of molecular gas $h=10 \mathrm{pc}$. The fit to the broad component gives FWHM $=61^{\prime}$, representing the scale height of bright sources in the disk distributed along the line of sight.

To examine the brightness distribution of point sources for a given flux, we plot the brightness distribution of $24 \mu \mathrm{m}$ sources as a function of longitude and present the results in three different panels in Figure 10. The flux of selected YSO candidates range between 0 and 5, 5 and 7, and 7 and 10 mag corresponding to bright, intermediate, and faint sources in the entire region of the $24 \mu \mathrm{m}$ survey but restricted only to within $b= \pm 10^{\prime}$. Such a tight latitude restriction most likely selects the sources that are populated in the Galactic plane. It is clear that the brightest $24 \mu \mathrm{m}$ sources show a stronger asymmetry in longitude, as shown in Figure 10(a), when compared to the distribution of fainter sources, as shown in Figures 10(b) and (c). The faintest stars dominate at greater longitudes but are not detected in the innermost region of the Galactic center. This is because of the confusion and the saturation caused by the extremely strong background. These distributions indicate that the brightest $24 \mu \mathrm{m}$ point sources exhibit an excess at negative longitudes similar to that seen in the candidate YSOs. The fainter $24 \mu \mathrm{m}$ sources seem to be uniformly distributed.

\section{THE SEDS OF CANDIDATE YSOs}

One of the difficulties in identifying YSOs by selecting them from [8]-[24] $\mu \mathrm{m}$ CMDs is the contamination by dusty and bright AGB stars. In addition, YSOs can populate left of the CMD line as well (as seen in Figures 6 and 7). In order to have more confidence in identifying YSO candidates and to characterize their properties, we obtained the SEDs of selected number of YSO candidates using measurements at eight wavelengths from $1.24 \mu \mathrm{m}$ to $24 \mu \mathrm{m}$ in order to determine the evolutionary phase of these protostellar candidates. The SED fitting technique is motivated by physical models and determines the physical properties of the sources. This technique is used for further refinement of the list of YSO candidates based on the CMD technique with its ad hoc distinctions in color. The SEDs of the sources are analyzed by comparing to a set of SEDs produced by a large grid of YSO models (Robitaille et al. 2006;
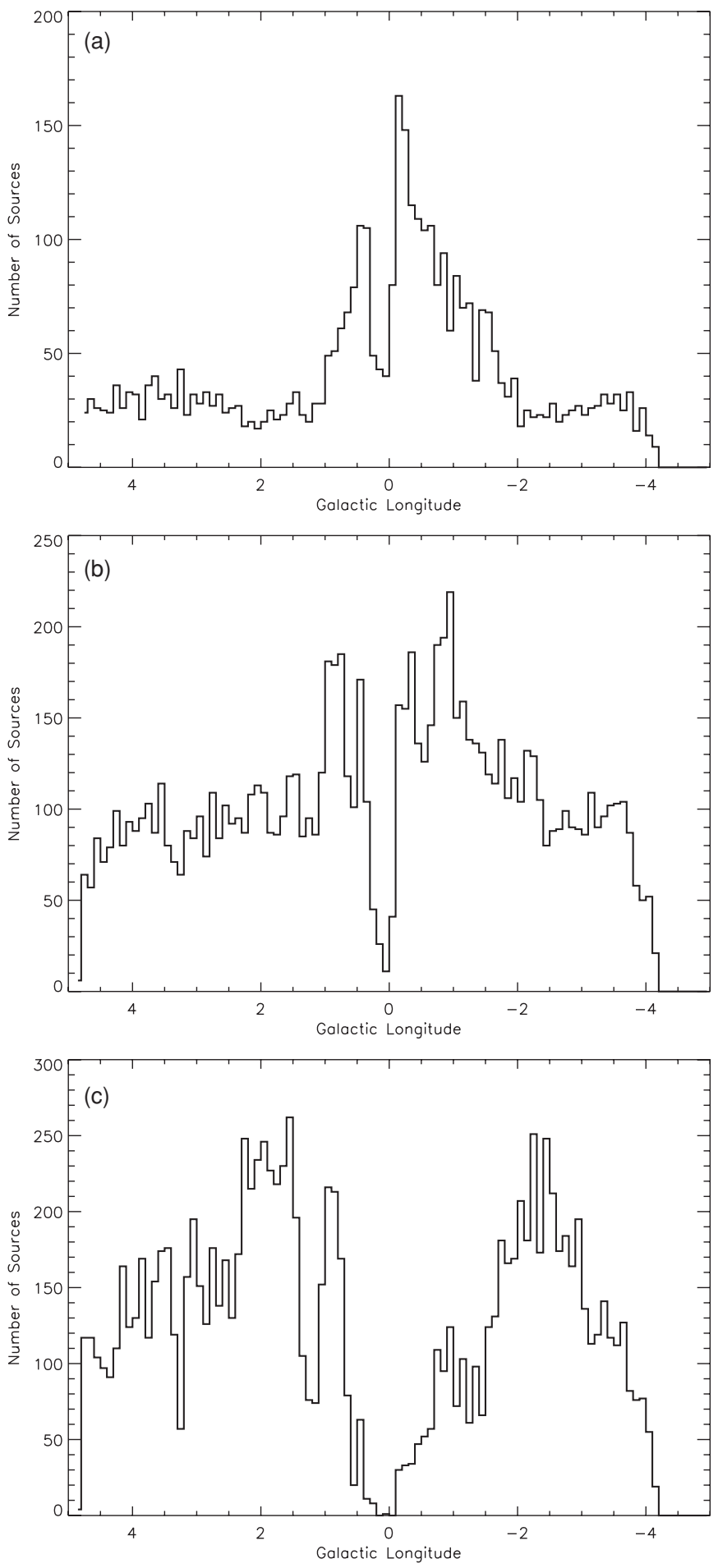

Figure 10. (a) Brightness distribution of $24 \mu \mathrm{m}$ point sources as a function of longitudes in shown for $24 \mu \mathrm{m}$ sources ranging between 0 and 5 mag. (b) The middle panel shows the distribution of point sources with intermediate brightness between 5 and 7 mag. (c) Similar to the middle panel except for faint sources between 7 and $10 \mathrm{mag}$. All the plots have used the entire region of the $24 \mu \mathrm{m}$ survey but restricted the latitudes to within $b= \pm 10^{\prime}$. The dips in the distribution near $l=0^{\circ}$ are largely caused by the inability to detect faint sources in regions where $24 \mu \mathrm{m}$ emission is extremely bright. The units of longitude are degrees.

Whitney et al. 2003a, 2003b). We use a linear regression fitter (Robitaille et al. 2007) to find all SEDs from the grid of models that are fit within a specified $\chi^{2}$ range to the data. The $\chi^{2}$ of the fit depends on the errors assumed for the data. We set 

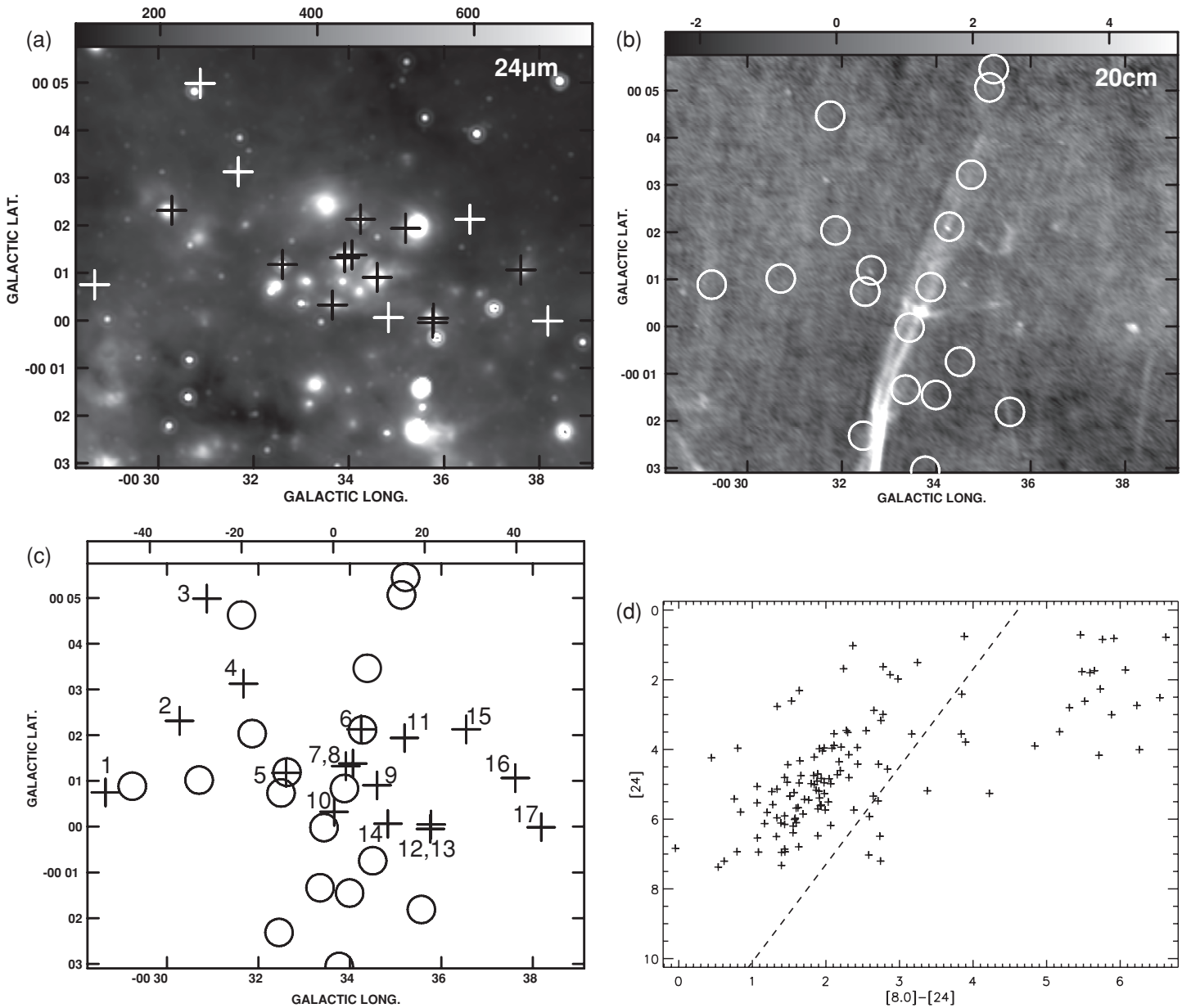

Figure 11. (a) Gray-scale $24 \mu \mathrm{m}$ image of the G359.43+0.02 stellar cluster. (b) A gray-scale $20 \mathrm{~cm}$ continuum image with a resolution of $12^{\prime \prime} \times 12^{\prime \prime}$ with an identical size to that shown in (a). (c) A finding chart of YSO candidate sources and radio continuum sources in the region shown in (a) and (b). (d) A plot of the CMD of the infrared sources found in (a). A total of 18 candidate YSOs are selected from the area to the right of the plot. The crosses present the position of the compact radio sources as listed in Table 1 whereas the circles coincide with the position of unsaturated $24 \mu \mathrm{m}$ YSO candidate sources, as listed in Table 2.

lower limits on the fractional flux error $(d F / F)$ to be $20 \%$ to account for variability between the 2MASS, IRAC, and MIPS observation dates. The minimum reduced $\chi^{2}$ for each source is determined. For all the models with a reduced $\chi^{2}$ within 1 of the minimum value, physical parameters corresponding to those models are averaged along with their standard deviations. The models are not always well constrained due to the incomplete SED information (many lower and upper limits and relatively few overall data points). However, this averaging is constrained by the finite extent of the grid of models.

We carried out our search for candidate YSOs toward two regions, using SED fitting of sources selected from CMDs. One is a small cluster of $24 \mu \mathrm{m}$ sources at G359.43+0.02 and the other is the candidate YSOs distributed within $|b|=10^{\prime}$ and $|l|=1.3$. We caution that the CMD selection biases us to redder sources. In addition, we have employed conservative errors in the SED fitting. Robitaille et al. (2006) defined three evolutionary stages based on their derived model properties, analogous to the classification based on the observed SED slope (Lada 1987): Stage I objects have envelope infall rate $\dot{M}_{\text {env }} / M_{\star}>10^{-6} \mathrm{yr}^{-1}$, Stage II objects have $\dot{M}_{\text {env }} / M_{\star}<$ $10^{-6} \mathrm{yr}^{-1}$ and $M_{\text {disk }} / M_{\star}>10^{-6}$, and Stage III objects have $\dot{M}_{\text {env }} / M_{\star}<10^{-6} \mathrm{yr}^{-1}$ and $M_{\text {disk }} / M_{\star}<10^{-6}$. That is, Stage I objects are young protostars embedded in an opaque infalling envelope. In Stage II objects the envelope has mostly dispersed, and the central star is surrounded by an opaque disk. In Stage
III the disk is optically thin. It is not clear if there is a longlived disk stage (Stage II) in high-mass sources, or if the disk is dispersed with the envelope. There are good reasons for the conservative (20\%) error bars, but it means that Stage II and III YSOs with smaller IR excesses may fail to be distinguished from reddened stellar atmospheres. It is clear that the YSO list will be incomplete and biased toward younger evolutionary stages, which are readily distinguishable from even dusty evolved stars. We discuss these two groups separately below and then estimate the SFR.

\subsection{G359.43+0.02 Cluster}

A striking cluster of $24 \mu \mathrm{m}$ sources lies about $8^{\prime}$ north of the Sgr C complex G359.4-0.1 with its thermal and nonthermal radio continuum components. Figures $11(\mathrm{a})$ and (b) show grayscale images of G359.43+0.02 at $24 \mu \mathrm{m}$ and $20 \mathrm{~cm}$, respectively. This cluster of $24 \mu \mathrm{m}$ source (G359.43+0.02) is distributed in the region where the northern extension of the nonthermal radio filament of $\mathrm{Sgr} \mathrm{C}$ dominates the radio continuum emission. In spite of strong extended background emission from the nonthermal filaments, there are several compact radio sources that are detected toward this cluster. The positions, the flux densities at 20 and $6 \mathrm{~cm}$ and the sizes of individual radio sources are listed in Table 1; Column 1 identifies the source number, Columns 2 and 3 show the Galactic coordinates, and Columns 4-7 give the flux densities in mJy and the source sizes 
Table 1

The Glactic Coordinates and Flux Density of Sources in G359.43+0.02

\begin{tabular}{|c|c|c|c|c|c|c|}
\hline No. & GLON & GLAT & $\mathrm{S}_{6 \mathrm{~cm}} \pm \sigma$ & $\mathrm{S}_{20 \mathrm{~cm}} \pm \sigma$ & $\theta_{\text {maj }}{ }^{\prime \prime}$ & $\theta_{\min }{ }^{\prime \prime}$ \\
\hline 1 & 359.522356 & 0.012511 & $6.5 \pm 1.6$ & $4.1 \pm 2.2$ & 8.8 & 2.0 \\
\hline 2 & 359.495378 & 0.038569 & $5.2 \pm 2.4$ & & 12.9 & 10.2 \\
\hline 3 & 359.485425 & 0.083103 & $6.5 \pm 3.6$ & $3.0 \pm 1.8$ & 7.9 & 3.8 \\
\hline 4 & 359.472092 & 0.052111 & $5.7 \pm 2.2$ & $5.3 \pm 2.4$ & 6.9 & 0.4 \\
\hline $5^{\mathrm{a}}$ & 359.456575 & 0.019586 & $3.6 \pm 1$ & $2.8 \pm 1.7$ & 4.2 & $\ldots$ \\
\hline $6^{b}$ & 359.429236 & 0.035489 & $3.4 \pm 1.2$ & $4.4 \pm 0.9$ & 5.8 & $\ldots$ \\
\hline 7 & 359.434825 & 0.022075 & $12.6 \pm 3.4$ & $\ldots$ & 19 & 11 \\
\hline 8 & 359.432242 & 0.022986 & $14.4 \pm 4$ & $\ldots$ & 21.3 & 11.1 \\
\hline 9 & 359.423431 & 0.015069 & $6.9 \pm 1.7$ & $4.8 \pm 2.5$ & 11 & 7 \\
\hline 10 & 359.439063 & 0.005402 & 76.0 & $96 \pm 9$ & $900 \operatorname{arcsec}^{2}$ & \\
\hline $11^{\mathrm{c}}$ & 359.413464 & 0.032297 & $1.4 \pm 1.7$ & $1.3 \pm 2$ & 11.1 & $\ldots$ \\
\hline $11 e^{c}$ & & & $49 \pm 20$ & $40.3 \pm 12$ & $1472 \operatorname{arcsec}^{2}$ & \\
\hline 12 & 359.403742 & 0.000797 & $13.8 \pm 2.8$ & $6.0 \pm 2.3$ & 13.4 & 11.6 \\
\hline 13 & 359.404019 & -0.000811 & $9.5 \pm 8.6$ & $9.7 \pm 5.2$ & 11.4 & 9.6 \\
\hline 14 & 359.419481 & 0.001042 & & & & \\
\hline 15 & 359.390961 & 0.035469 & $4.3 \pm 1.3$ & $9.5 \pm 4.2$ & 8.4 & 5.9 \\
\hline 16 & 359.373078 & 0.017686 & & $\ldots$ & 9.0 & 8.2 \\
\hline 17 & 359.363663 & -0.000190 & $25 \pm 3.2$ & $30.8 \pm 5.6$ & & \\
\hline
\end{tabular}

Notes.

a Infrared counterpart 300758

b Infrared counterpart 284291.

c Source 11 has a central compact source and an extended component

at 6 and $20 \mathrm{~cm}$, respectively. These values are estimated by twodimensional Gaussian fits to individual compact radio sources at 6 and $20 \mathrm{~cm}$. The finding chart for the radio and $24 \mu \mathrm{m}$ sources is shown in Figure 11(c). The crosses drawn on Figures 11(a) and (c) show the positions of radio continuum point sources whereas the circles drawn on Figure 11(b) and (c) show the position of compact $24 \mu \mathrm{m}$ sources. Because of the saturation of several $24 \mu \mathrm{m}$ sources in this cluster, there are only two radio sources, as listed in Table 1, that have cataloged $24 \mu \mathrm{m}$ counterparts.

The CMD covered by the region shown in Figures 11(a) and (b) is shown in Figure 11(d). A total of 28 candidate YSOs is detected in the YSO candidate region of Figure 11(d). We selected these candidate YSOs and fitted their SEDs. The SED fitting leads to the rejection of 10 sources as YSO candidates. Thus, the total number of YSO fitted sources is about $64 \%$ of the sources that are selected from CMDs. The fraction of Stage I objects is $78 \%$ of the total number of SED fitted sources.

The rejected sources are likely to be evolved stellar sources, for which the model YSO SEDs give poor fits. Other reasons for rejecting sources could be (1) variability between IRAC and MIPS, (2) errors in photometry, (3) source confusion where multiple sources contribute and give an SED that is not well fit by a single YSO model, (4) bad extraction of data, or (5) there are no correct models in the grid.

Figure 12(a) shows the fitted SEDs for each individual YSO candidate source and Table 2 shows the physical characteristics derived from their SED fitting. These sources are assumed to be $8.5 \mathrm{kpc}$ away with $A_{v}$ values ranging between 5 and $50 \mathrm{mag}$. Given that 9 out of 18 of these have fewer than five data points, we note from Figure 12(a) that the flux of most cluster members is elevated at $24 \mu \mathrm{m}$ when compared to IRAC and 2MASS data points. This supports the identification of these sources as YSOs. The parameters of the fits to these 18 sources suggest that the masses of the individual protostars range between 5 and $11.7 M_{\odot}$ with corresponding luminosities ranging between $6.5 \times 10^{2}$ and $10^{4} L_{\odot}$.

Columns 1-10 of Tables 2, 3, and 4 show (1) the SST (Spitzer Space Telescope) Galactic center (GC) number based on the IRAC catalogs (Ramirez et al. 2008), (2 and 3) Galactic coordinates, (4) $\chi^{2}$ values, (5) number of acceptably fitting models "nfits," (6) the visual extinction $A_{v}$, (7 and 8) the average mass and luminosity of the protostar, (9) the average mass accretion rate which includes their corresponding standard deviations, and (10) the evolutionary stage of the fitted sources. The error bars are $1 \sigma$ standard deviations of the well-fit values. The standard deviation of the fitted values reflect the limited number of data points that went into a fit. Thus, additional data points in other wavelength bands should improve the reliability in a fit. We examined $\chi^{2}$ with values ranging between 0.5 and 2 . There were many fits that did not go through the data points when a value of 2 was selected. On the other hand, when we used a value of 0.5 , there were not enough fits. Thus, we visually selected fits with $\chi^{2} \sim 1, A_{v}$ values ranging between 5 and 50 mag. $A_{v}$ restriction is unlikely to bias the results. It is restricting some of the less likely models from being included in the fitting. For example, if the $A_{v}$ to a source is 10 , and the fitter finds a model with $A_{v}=0$, it is going to find a more embedded model than it should in order to fit the high extinction. By selecting a reasonable range of $A_{v}$, we are getting more appropriate YSO models to fit the source.

\subsection{Sources within $|b|=10^{\prime}$}

The number of candidate YSOs, as shown to the right in the CMDs of Figure 7(a) is 347 and 212 distributed at negative and positive longitudes, respectively. These numbers are obviously lower limits due to the bright saturated background at $24 \mu \mathrm{m}$. SED fitting showed a total of 213,112 , and 35 for Stage I, II, and III sources. We note that $\sim 60 \%$ of YSO candidates are identified as Stage I sources. When grouped by longitude, the total numbers of Stage I, II, and III sources are 131, 69, 23 at $l<0^{\circ}$ whereas 82, 43, 12 sources are distributed at $l>0^{\circ}$, respectively. The total number of acceptable sources that are SED fitted within the restricted region is 360 . This number is $\sim 64 \%$ of the total number of sources that are selected from CMDs. A similar fraction of SED fitted stars is found in 

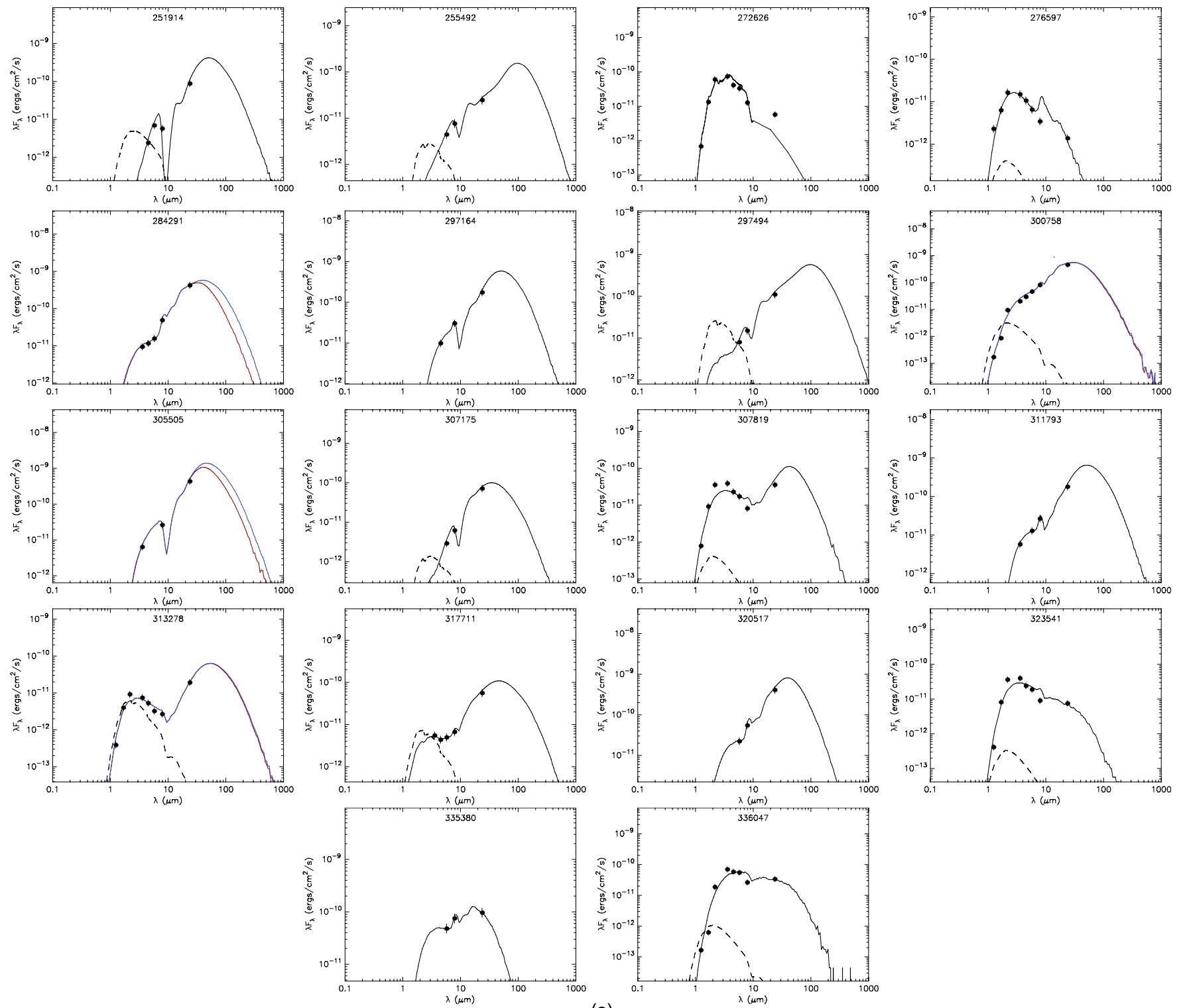

(a)

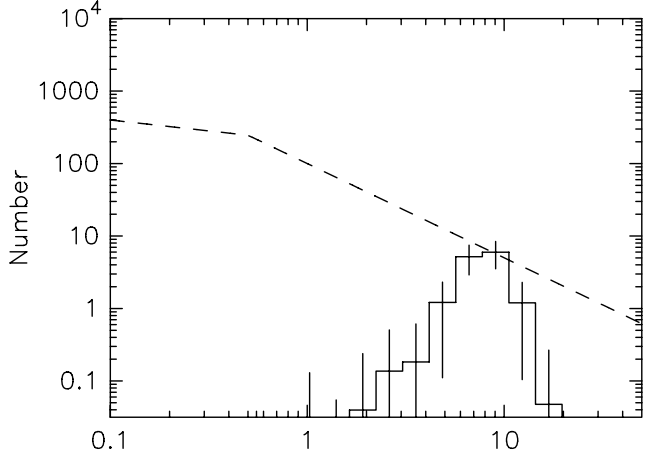

(b) Mass $\left(M_{\odot}\right)$

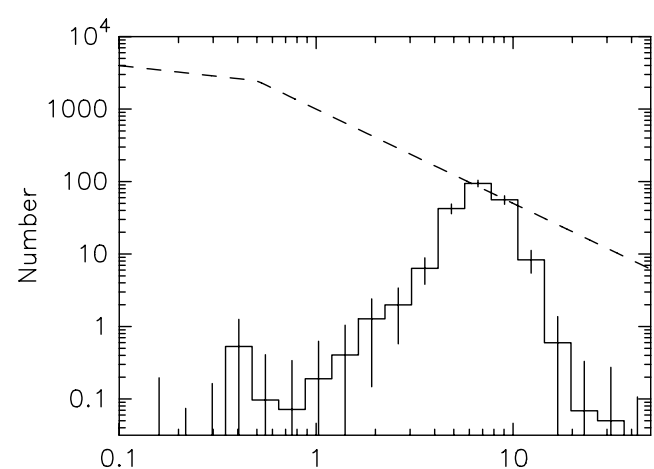

(c) Mass $\left(M_{\odot}\right)$

Figure 12. (a) SED fitted plots of 18 candidate YSOs found in the G359.43+0.02 cluster. The parameters of the fits are shown in Table 2. (b) The histogram of the mass of YSO candidates for the cluster G359.43+0.02. (c) Similar to (b) except for all the YSO candidates distributed within the Galactic disk restricted to $|b|=10^{\prime}$ and $|l|<1$. 3. "Number" in both (b) and (c) represent all the good fits for a given source in the distribution and is normalized so that the sum equals the average mass which is given in Tables 2-4. Each source is represented by a distribution of well fit masses. The broken power-law form is fitted to the data and is shown as dashed line in both (b) and (c).

(A color version of this figure is available in the online journal.) 
0
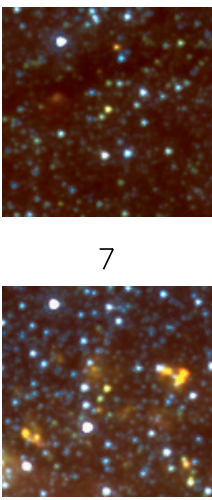

14

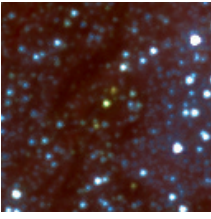

21

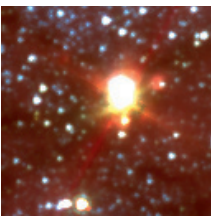

28

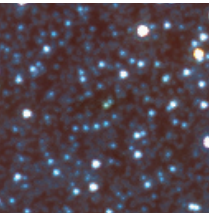

1

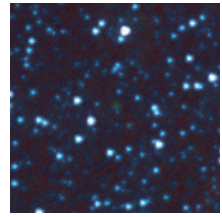

8

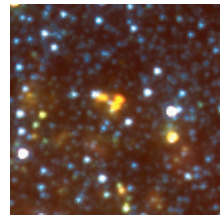

15

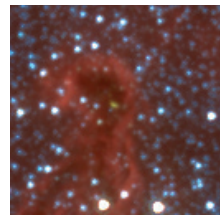

22

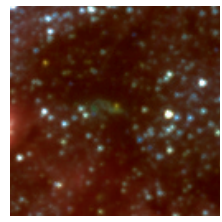

29

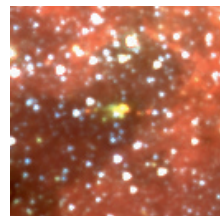

2

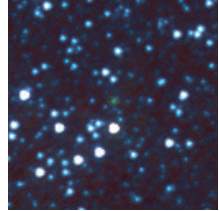

9

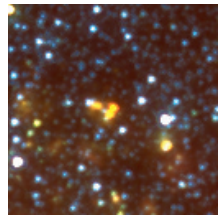

16

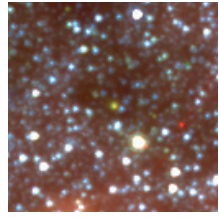

23

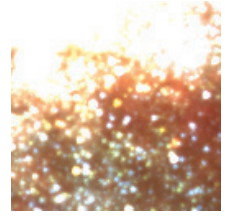

30

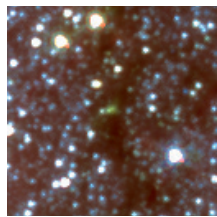

3

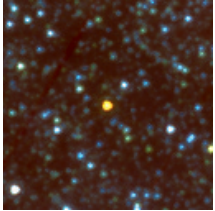

10

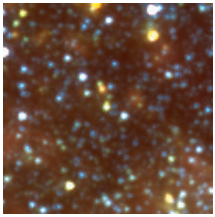

17

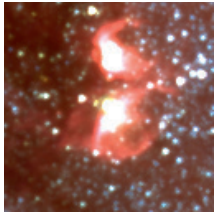

24

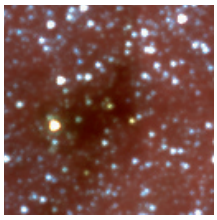

31

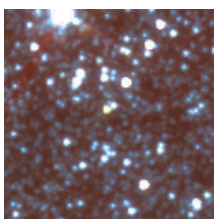

4

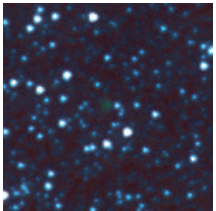

11

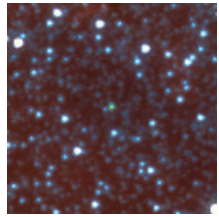

18

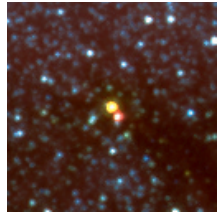

25

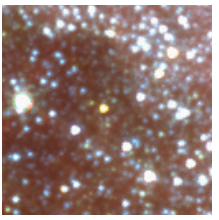

32

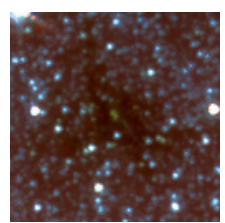

5

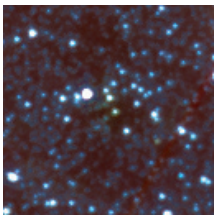

12

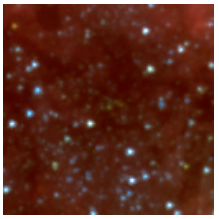

19

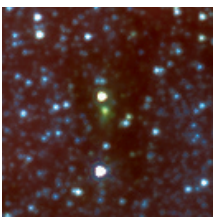

26

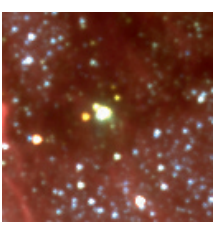

6

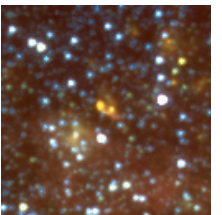

13

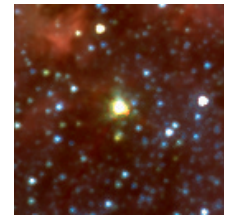

20

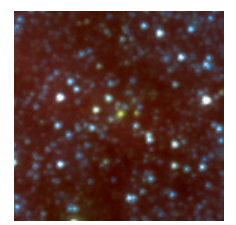

27

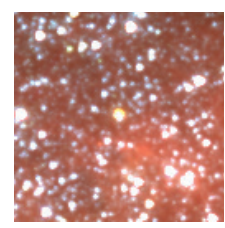

Figure 13. Color images of all the $4.5 \mu \mathrm{m}$ sources found in the IRAC survey region. Red, green, and blue colors denote $8,4.5$, and $3.6 \mu \mathrm{m}$ emission, respectively. Each image shows a region of 7.5 7.5 centered on the $4.5 \mu \mathrm{m}$ excess sources.

(A color version of this figure is available in the online journal.)

Table 2

The Parameters of the SED Fits to Candidate YSOs in G359.43+0.02

\begin{tabular}{lccccccccc}
\hline \hline SST GC No. & $l$ & $b$ & $\chi_{\min }^{2}$ & nfits & $\left\langle A_{v}\right\rangle$ & $\left\langle M_{\star}\right\rangle$ & $\left\langle L_{\star}\right\rangle$ & $\left\langle\dot{M}_{\text {env }}\right\rangle$ & $\langle$ Stage $\rangle$ \\
\hline 251914 & 359.4130 & 0.09095 & 0.03 & 310 & 32.1 & $8.0 \pm 1.3$ & $1.4 \pm 1.2 \mathrm{E}+03$ & $2.7 \pm 4.3 \mathrm{E}-04$ & $\mathrm{I}$ \\
255492 & 359.4145 & 0.08451 & 0.00 & 736 & 31.6 & $5.0 \pm 1.6$ & $6.5 \pm 7.8 \mathrm{E}+02$ & $1.1 \pm 3.5 \mathrm{E}-04$ & I \\
272626 & 359.4288 & 0.05876 & 4.57 & 240 & 18.8 & $10.1 \pm 0.7$ & $6.5 \pm 1.2 \mathrm{E}+03$ & $0.0 \pm 0.0 \mathrm{E}+00$ & II \\
276597 & 359.4709 & 0.07663 & 5.97 & 52 & 15.0 & $7.5 \pm 0.5$ & $2.3 \pm 0.7 \mathrm{E}+03$ & $0.0 \pm 0.0 \mathrm{E}+00$ & III \\
$284291^{\mathrm{a}}$ & 359.4287 & 0.03534 & 0.07 & 204 & 37.6 & $9.5 \pm 1.0$ & $5.2 \pm 1.9 \mathrm{E}+03$ & $0.8 \pm 3.8 \mathrm{E}-04$ & $\mathrm{I}$ \\
297164 & 359.4352 & 0.01400 & 0.00 & 739 & 36.2 & $8.4 \pm 0.9$ & $2.4 \pm 1.2 \mathrm{E}+03$ & $1.3 \pm 3.5 \mathrm{E}-04$ & I \\
297494 & 359.4688 & 0.03400 & 0.00 & 703 & 34.9 & $7.5 \pm 0.9$ & $1.7 \pm 1.0 \mathrm{E}+03$ & $1.7 \pm 4.6 \mathrm{E}-04$ & I \\
$300758^{\mathrm{b}}$ & 359.4562 & 0.01993 & 1.66 & 59 & 17.6 & $8.8 \pm 1.1$ & $3.3 \pm 1.6 \mathrm{E}+03$ & $5.1 \pm 5.2 \mathrm{E}-05$ & I \\
305505 & 359.4583 & 0.01236 & 0.00 & 837 & 38.0 & $10.1 \pm 1.4$ & $5.7 \pm 2.2 \mathrm{E}+03$ & $2.2 \pm 5.1 \mathrm{E}-04$ & I \\
307175 & 359.4427 & 0.02637 & 0.00 & 443 & 36.1 & $6.9 \pm 1.0$ & $1.2 \pm 0.9 \mathrm{E}+03$ & $2.2 \pm 5.5 \mathrm{E}-04$ & I \\
307819 & 359.4249 & 0.01234 & 4.18 & 20 & 15.0 & $6.2 \pm 0.6$ & $1.1 \pm 0.1 \mathrm{E}+03$ & $1.0 \pm 4.7 \mathrm{E}-04$ & I \\
311793 & 359.4072 & 0.03009 & 0.00 & 453 & 40.8 & $8.3 \pm 0.8$ & $2.8 \pm 1.2 \mathrm{E}+03$ & $1.0 \pm 3.4 \mathrm{E}-04$ & $\mathrm{I}$ \\
313278 & 359.4882 & 0.01704 & 1.28 & 18 & 16.8 & $6.3 \pm 0.7$ & $2.9 \pm 0.8 \mathrm{E}+02$ & $1.8 \pm 2.3 \mathrm{E}-04$ & I \\
317711 & 359.4334 & 0.02412 & 0.08 & 450 & 24.7 & $6.5 \pm 0.8$ & $8.8 \pm 5.9 \mathrm{E}+02$ & $1.0 \pm 2.9 \mathrm{E}-04$ & I \\
320517 & 359.4441 & 0.02219 & 0.00 & 784 & 39.2 & $9.6 \pm 1.1$ & $5.1 \pm 1.8 \mathrm{E}+03$ & $1.4 \pm 6.3 \mathrm{E}-04$ & I \\
323541 & 359.5125 & 0.01483 & 5.03 & 55 & 16.6 & $7.0 \pm 1.0$ & $2.0 \pm 0.8 \mathrm{E}+03$ & $0.0 \pm 0.0 \mathrm{E}+00$ & II \\
335380 & 359.4371 & 0.05085 & 0.00 & 346 & 30.2 & $9.5 \pm 2.4$ & $5.8 \pm 4.6 \mathrm{E}+03$ & $2.3 \pm 9.5 \mathrm{E}-04$ & I \\
336047 & 359.4591 & 0.03848 & 7.85 & 6 & 17.0 & $11.7 \pm 0.8$ & $1.0 \pm 0.2 \mathrm{E}+04$ & $0.0 \pm 0.0 \mathrm{E}+00$ & II \\
\hline
\end{tabular}

Notes.

a Radio counterpart 6.

${ }^{\mathrm{b}}$ Radio counterpart 5 . 
Table 3

The Parameters of the SED Fits to Candidate YSOs in $l<0^{\circ}$

\begin{tabular}{|c|c|c|c|c|c|c|c|c|c|}
\hline SST GC No. & $l$ & $b$ & $\chi_{\min }^{2}$ & nfits & $\left\langle A_{v}\right\rangle$ & $\left\langle M_{\star}\right\rangle$ & $\left\langle L_{\star}\right\rangle$ & $\left\langle\dot{M}_{\text {env }}\right\rangle$ & $\langle$ Stage $\rangle$ \\
\hline 116907 & 358.7362 & 0.0045 & 0.80 & 13 & 16.3 & $7.7 \pm 0.5$ & $1.2 \pm 0.3 \mathrm{E}+03$ & $7.0 \pm 9.5 \mathrm{E}-04$ & $\mathrm{I}$ \\
\hline 119577 & 358.8940 & 0.0943 & 18.80 & 3 & 15.0 & $7.0 \pm 0.0$ & $4.7 \pm 0.1 \mathrm{E}+02$ & $2.6 \pm 3.5 \mathrm{E}-04$ & I \\
\hline 119780 & 358.8605 & 0.0729 & 0.07 & 471 & 29.7 & $4.7 \pm 0.8$ & $4.2 \pm 3.3 \mathrm{E}+02$ & $0.2 \pm 1.8 \mathrm{E}-04$ & I \\
\hline 121566 & 358.8108 & 0.0369 & 5.64 & 10 & 15.5 & $6.9 \pm 0.0$ & $1.5 \pm 0.0 \mathrm{E}+03$ & $0.0 \pm 0.0 \mathrm{E}-00$ & III \\
\hline 122375 & 358.7448 & -0.0062 & 0.00 & 1072 & 28.1 & $4.1 \pm 1.6$ & $2.5 \pm 2.7 \mathrm{E}+02$ & $0.7 \pm 1.7 \mathrm{E}-04$ & I \\
\hline 126327 & 358.8288 & 0.0347 & 7.12 & 6 & 15.0 & $7.0 \pm 0.1$ & $4.9 \pm 0.4 \mathrm{E}+02$ & $3.1 \pm 3.3 \mathrm{E}-04$ & I \\
\hline 127127 & 358.7587 & -0.0108 & 0.00 & 485 & 34.7 & $5.1 \pm 1.5$ & $0.8 \pm 1.0 \mathrm{E}+03$ & $0.3 \pm 2.8 \mathrm{E}-09$ & II \\
\hline 127297 & 358.7373 & -0.0245 & 0.00 & 1537 & 38.9 & $8.9 \pm 1.0$ & $3.4 \pm 1.4 \mathrm{E}+03$ & $1.1 \pm 3.0 \mathrm{E}-04$ & I \\
\hline 131902 & 358.9174 & 0.0747 & 17.20 & 12 & 15.0 & $9.5 \pm 0.4$ & $5.3 \pm 0.8 \mathrm{E}+03$ & $0.0 \pm 0.0 \mathrm{E}-00$ & III \\
\hline 132101 & 358.9409 & 0.0887 & 0.23 & 71 & 39.4 & $12.6 \pm 3.1$ & $1.0 \pm 0.5 \mathrm{E}+04$ & $0.9 \pm 1.7 \mathrm{E}-03$ & I \\
\hline 134327 & 358.9658 & 0.0982 & 0.07 & 84 & 15.7 & $5.0 \pm 0.5$ & $3.5 \pm 0.9 \mathrm{E}+02$ & $1.2 \pm 4.2 \mathrm{E}-05$ & I \\
\hline 136092 & 358.9102 & 0.0592 & 0.22 & 126 & 18.1 & $8.5 \pm 1.2$ & $4.0 \pm 1.8 \mathrm{E}+03$ & $3.2 \pm 9.4 \mathrm{E}-05$ & I \\
\hline 136259 & 358.8728 & 0.0356 & 1.05 & 84 & 15.9 & $7.1 \pm 1.0$ & $1.6 \pm 1.3 \mathrm{E}+03$ & $1.2 \pm 4.1 \mathrm{E}-04$ & I \\
\hline 137737 & 358.8181 & -0.0020 & 11.70 & 5 & 15.0 & $6.5 \pm 0.2$ & $2.9 \pm 0.4 \mathrm{E}+02$ & $1.3 \pm 0.5 \mathrm{E}-04$ & I \\
\hline 142082 & 358.8714 & 0.0194 & 7.02 & 4 & 15.0 & $6.8 \pm 0.1$ & $3.6 \pm 0.4 \mathrm{E}+02$ & $2.3 \pm 3.0 \mathrm{E}-04$ & I \\
\hline 142930 & 358.8283 & -0.0094 & 5.72 & 5 & 15.0 & $6.6 \pm 0.0$ & $4.6 \pm 0.0 \mathrm{E}+02$ & $1.7 \pm 0.0 \mathrm{E}-00$ & I \\
\hline 142942 & 358.9795 & 0.0840 & 0.11 & 58 & 21.9 & $7.6 \pm 0.6$ & $7.3 \pm 3.4 \mathrm{E}+02$ & $2.5 \pm 2.7 \mathrm{E}-04$ & I \\
\hline 146060 & 358.8148 & -0.0260 & 0.00 & 646 & 37.5 & $5.4 \pm 1.9$ & $1.0 \pm 1.4 \mathrm{E}+03$ & $5.2 \pm 2.3 \mathrm{E}-04$ & I \\
\hline 147176 & 358.7761 & -0.0527 & 10.72 & 2 & 15.0 & $6.3 \pm 0.0$ & $2.5 \pm 0.0 \mathrm{E}+02$ & $1.8 \pm 0.0 \mathrm{E}-04$ & I \\
\hline 147584 & 358.8293 & -0.0210 & 0.00 & 790 & 28.9 & $7.4 \pm 1.5$ & $2.2 \pm 1.5 \mathrm{E}+03$ & $0.6 \pm 2.4 \mathrm{E}-04$ & I \\
\hline 147743 & 358.7765 & -0.0540 & 12.29 & 2 & 15.0 & $6.3 \pm 0.0$ & $2.5 \pm 0.0 \mathrm{E}+02$ & $1.8 \pm 0.0 \mathrm{E}-04$ & I \\
\hline 150660 & 358.8131 & -0.0391 & 1.65 & 18 & 15.5 & $5.6 \pm 0.2$ & $2.1 \pm 0.3 \mathrm{E}+02$ & $1.1 \pm 1.0 \mathrm{E}-05$ & I \\
\hline 153970 & 358.8569 & -0.0206 & 5.57 & 27 & 15.0 & $8.3 \pm 0.7$ & $3.3 \pm 1.0 \mathrm{E}+03$ & $0.0 \pm 0.0 \mathrm{E}-00$ & III \\
\hline 158032 & 358.8088 & -0.0607 & 4.76 & 50 & 15.0 & $4.4 \pm 0.3$ & $3.2 \pm 0.9 \mathrm{E}+02$ & $0.0 \pm 0.0 \mathrm{E}-00$ & II \\
\hline 158837 & 358.8657 & -0.0277 & 0.38 & 174 & 17.0 & $4.2 \pm 0.4$ & $2.7 \pm 0.9 \mathrm{E}+02$ & $0.3 \pm 3.4 \mathrm{E}-08$ & II \\
\hline 161727 & 358.9833 & 0.0373 & 0.00 & 415 & 37.9 & $9.0 \pm 1.3$ & $3.5 \pm 1.8 \mathrm{E}+03$ & $2.6 \pm 8.1 \mathrm{E}-04$ & I \\
\hline 165041 & 358.9781 & 0.0254 & 15.11 & 3 & 15.0 & $6.7 \pm 0.2$ & $4.5 \pm 4.0 \mathrm{E}+02$ & $2.6 \pm 3.5 E-04$ & I \\
\hline 166903 & 358.8068 & -0.0850 & 0.00 & 683 & 34.7 & $6.5 \pm 1.8$ & $1.8 \pm 2.1 \mathrm{E}+03$ & $0.7 \pm 3.5 \mathrm{E}-04$ & I \\
\hline 168737 & 359.0166 & 0.0399 & 6.03 & 507 & 15.0 & $9.7 \pm 0.5$ & $5.7 \pm 1.0 \mathrm{E}+03$ & $0.0 \pm 0.0 \mathrm{E}-00$ & III \\
\hline 170621 & 358.7689 & -0.1181 & 5.02 & 3 & 15.0 & $5.5 \pm 0.0$ & $1.5 \pm 0.0 \mathrm{E}+02$ & $7.1 \pm 0.0 \mathrm{E}-05$ & I \\
\hline 173766 & 358.9977 & 0.0149 & 2.62 & 101 & 15.5 & $3.7 \pm 0.9$ & $7.3 \pm 2.2 \mathrm{E}+01$ & $0.7 \pm 1.0 \mathrm{E}-04$ & I \\
\hline 175663 & 359.2416 & 0.1607 & 8.10 & 19 & 17.1 & $7.0 \pm 0.7$ & $7.0 \pm 2.3 \mathrm{E}+02$ & $1.2 \pm 2.0 \mathrm{E}-04$ & I \\
\hline 179253 & 359.2140 & 0.1343 & 1.73 & 55 & 15.5 & $5.3 \pm 0.5$ & $6.7 \pm 2.4 \mathrm{E}+02$ & $0.0 \pm 0.0 \mathrm{E}-00$ & II \\
\hline 180155 & 358.8542 & -0.0901 & 0.00 & 727 & 27.2 & $4.1 \pm 0.7$ & $2.3 \pm 1.6 \mathrm{E}+02$ & $0.7 \pm 4.1 \mathrm{E}-05$ & I \\
\hline 187849 & 358.8233 & -0.1286 & 6.13 & 10 & 15.0 & $5.7 \pm 0.4$ & $1.8 \pm 0.4 \mathrm{E}+02$ & $2.7 \pm 2.6 \mathrm{E}-04$ & I \\
\hline 188031 & 358.9874 & -0.0278 & 7.34 & 5 & 15.0 & $8.2 \pm 0.2$ & $9.9 \pm 1.9 \mathrm{E}+02$ & $5.9 \pm 8.9 \mathrm{E}-04$ & I \\
\hline 188899 & 358.8238 & -0.1308 & 8.31 & 3 & 15.0 & $5.5 \pm 0.0$ & $1.5 \pm 0.0 \mathrm{E}+02$ & $7.1 \pm 0.0 \mathrm{E}-05$ & I \\
\hline 189586 & 359.1190 & 0.0497 & 0.00 & 303 & 28.6 & $4.5 \pm 1.7$ & $5.1 \pm 6.8 \mathrm{E}+02$ & $1.4 \pm 3.1 \mathrm{E}-04$ & I \\
\hline 193986 & 359.0376 & -0.0106 & 8.56 & 28 & 15.0 & $9.4 \pm 0.6$ & $5.1 \pm 1.0 \mathrm{E}+03$ & $0.0 \pm 0.0 \mathrm{E}-00$ & II \\
\hline 194367 & 358.8665 & -0.1171 & 0.00 & 106 & 36.1 & $7.6 \pm 3.3$ & $2.9 \pm 7.2 \mathrm{E}+03$ & $4.0 \pm 9.9 \mathrm{E}-04$ & I \\
\hline 194640 & 358.9834 & -0.0455 & 3.29 & 6 & 15.0 & $6.9 \pm 0.1$ & $4.8 \pm 0.2 \mathrm{E}+02$ & $3.5 \pm 3.0 \mathrm{E}-04$ & I \\
\hline 198456 & 359.0938 & 0.0138 & 6.5 & 88 & 16 & $5.8 \pm 0.7$ & $9.1 \pm 3$ & $0.0 \pm 0.0 \mathrm{E}-$ & II \\
\hline 201854 & 359.2106 & 0.0782 & 5.57 & 46 & 15.0 & $4.2 \pm 0.4$ & $2.5 \pm 0.8 \mathrm{E}+02$ & $2.0 \pm 9.5 \mathrm{E}-06$ & II \\
\hline 202699 & 359.1513 & 0.0397 & 8.60 & 189 & 15.0 & $9.3 \pm 0.6$ & $5.1 \pm 1.2 \mathrm{E}+03$ & $0.0 \pm 0.0 \mathrm{E}-00$ & III \\
\hline 204187 & 358.9588 & -0.0824 & 8.50 & 1 & 15.0 & $6.7 \pm 0.0$ & $3.2 \pm 0.0 \mathrm{E}+02$ & $4.8 \pm 0.0 \mathrm{E}-05$ & I \\
\hline 204714 & 359.0956 & 0.0007 & 2.13 & 51 & 15.0 & $4.6 \pm 0.7$ & $4.0 \pm 3.4 \mathrm{E}+02$ & $0.2 \pm 1.3 \mathrm{E}-05$ & II \\
\hline 206043 & 359.3542 & 0.1572 & 3.38 & 31 & 15.0 & $6.2 \pm 0.5$ & $1.0 \pm 0.3 \mathrm{E}+03$ & $0.0 \pm 0.0 \mathrm{E}-00$ & III \\
\hline 207736 & 358.9448 & -0.0992 & 2.03 & 14 & 15.2 & $6.6 \pm 0.4$ & $3.4 \pm 0.7 \mathrm{E}+02$ & $2.3 \pm 2.0 \mathrm{E}-04$ & I \\
\hline 208385 & 359.3569 & 0.1534 & 4.00 & 17 & 15.0 & $4.6 \pm 0.4$ & $1.7 \pm 0.3 \mathrm{E}+02$ & $1.3 \pm 2.1 \mathrm{E}-05$ & I \\
\hline 212351 & 359.1824 & 0.0368 & 1.48 & 2 & 15.3 & $6.7 \pm 0.0$ & $3.2 \pm 0.0 \mathrm{E}+02$ & $4.8 \pm 0.0 \mathrm{E}-05$ & I \\
\hline 212533 & 358.8852 & -0.1468 & 21.18 & 4 & 15.0 & $6.9 \pm 0.1$ & $3.9 \pm 0.5 \mathrm{E}+02$ & $4.1 \pm 3.5 \mathrm{E}-04$ & I \\
\hline 214283 & 359.0873 & -0.0262 & 0.15 & 407 & 19.2 & $5.0 \pm 0.9$ & $5.6 \pm 9.2 \mathrm{E}+02$ & $0.5 \pm 6.5 \mathrm{E}-05$ & I \\
\hline 214291 & 358.9865 & -0.0883 & 5.69 & 36 & & & $1.2 \pm 0.1 \mathrm{E}+03$ & $0 \mathrm{E}-00$ & III \\
\hline 214384 & 359.0151 & -0.0708 & 1.51 & 4 & 15.0 & $6.6 \pm 0.0$ & $4.6 \pm 0.0 \mathrm{E}+02$ & $1.7 \pm 0.0 \mathrm{E}-05$ & I \\
\hline 215021 & 359.0938 & -0.0238 & 7.90 & 73 & 15.0 & $6.5 \pm 0.2$ & $1.2 \pm 0.1 \mathrm{E}+03$ & $0.0 \pm 0.0 \mathrm{E}-00$ & III \\
\hline 215431 & 359.1431 & 0.0056 & 6.00 & 3 & 15.0 & $5.5 \pm 0.0$ & $1.5 \pm 0.0 \mathrm{E}+02$ & $7.1 \pm 0.0 \mathrm{E}-05$ & I \\
\hline 216691 & 359.3706 & 0.1428 & 2.42 & 99 & 21.3 & $7.9 \pm 1.4$ & $3.1 \pm 2.5 \mathrm{E}+03$ & $0.0 \pm 0.0 \mathrm{E}-00$ & II \\
\hline 219986 & 359.1208 & -0.0185 & 0.01 & 471 & 36.0 & $7.7 \pm 1.1$ & $1.2 \pm 0.7 \mathrm{E}+03$ & $2.3 \pm 4.7 \mathrm{E}-04$ & I \\
\hline 223624 & 359.0653 & -0.0610 & 9.33 & 56 & 15.4 & $9.4 \pm 0.8$ & $5.2 \pm 1.3 \mathrm{E}+03$ & $0.0 \pm 0.0 \mathrm{E}-00$ & II \\
\hline 224770 & 359.4100 & 0.1489 & 0.54 & 219 & 16.2 & $6.6 \pm 0.8$ & $1.4 \pm 0.5 \mathrm{E}+03$ & $0.0 \pm 0.0 \mathrm{E}-00$ & II \\
\hline 225677 & 359.1460 & -0.0158 & 5.60 & 4 & 15.0 & $6.4 \pm 0.0$ & $4.1 \pm 0.0 \mathrm{E}+02$ & $7.0 \pm 0.0 \mathrm{E}-05$ & I \\
\hline 229216 & 359.0992 & -0.0525 & 0.98 & 91 & 15.1 & $4.6 \pm 0.5$ & $2.4 \pm 1.0 \mathrm{E}+02$ & $2.5 \pm 9.5 \mathrm{E}-05$ & I \\
\hline 231270 & 359.3173 & 0.0771 & 0.00 & 637 & 29.8 & $7.3 \pm 1.6$ & $2.2 \pm 1.9 \mathrm{E}+03$ & $1.3 \pm 5.7 \mathrm{E}-04$ & I \\
\hline 235473 & 359.4716 & 0.1627 & 0.01 & 683 & 28.7 & $5.9 \pm 1.2$ & $1.1 \pm 0.9 \mathrm{E}+03$ & $0.1 \pm 1.5 \mathrm{E}-04$ & I \\
\hline 235995 & 359.0383 & -0.1052 & 9.89 & 183 & 15.0 & $10.6 \pm 0.1$ & $7.5 \pm 0.3 \mathrm{E}+03$ & $0.0 \pm 0.0 \mathrm{E}-00$ & III \\
\hline 237228 & 359.1848 & -0.0177 & 20.81 & 4 & 15.0 & $8.5 \pm 0.0$ & $3.6 \pm 0.0 \mathrm{E}+03$ & $0.0 \pm 0.0 \mathrm{E}-00$ & II \\
\hline
\end{tabular}


Table 3

(Continued)

\begin{tabular}{|c|c|c|c|c|c|c|c|c|c|}
\hline SST GC No. & $l$ & $b$ & $\chi_{\min }^{2}$ & nfits & $\left\langle A_{v}\right\rangle$ & $\left\langle M_{\star}\right\rangle$ & $\left\langle L_{\star}\right\rangle$ & $\left\langle\dot{M}_{\text {env }}\right\rangle$ & $\langle$ Stage $\rangle$ \\
\hline 238167 & 359.4078 & 0.1174 & 2.40 & 78 & 15.3 & $7.9 \pm 0.7$ & $2.9 \pm 1.1 \mathrm{E}+03$ & $0.0 \pm 0.0 \mathrm{E}-00$ & III \\
\hline 241266 & 359.2441 & 0.0096 & 6.91 & 53 & 15.0 & $6.9 \pm 0.5$ & $1.6 \pm 0.4 \mathrm{E}+03$ & $0.0 \pm 0.0 \mathrm{E}-00$ & III \\
\hline 243433 & 359.4619 & 0.1388 & 6.30 & 65 & 15.0 & $5.0 \pm 0.3$ & $4.9 \pm 1.2 \mathrm{E}+02$ & $0.0 \pm 0.0 \mathrm{E}-00$ & II \\
\hline 243522 & 359.1801 & -0.0348 & 0.51 & 20 & 15.1 & $5.9 \pm 0.4$ & $4.4 \pm 2.0 \mathrm{E}+02$ & $3.7 \pm 6.9 \mathrm{E}-05$ & I \\
\hline 246410 & 359.3009 & 0.0334 & 2.57 & 15 & 18.1 & $8.8 \pm 0.8$ & $1.8 \pm 0.4 \mathrm{E}+03$ & $2.0 \pm 1.7 \mathrm{E}-04$ & I \\
\hline 251494 & 359.1027 & -0.0992 & 6.36 & 10 & 15.0 & $7.1 \pm 0.2$ & $1.8 \pm 0.2 \mathrm{E}+03$ & $0.0 \pm 0.0 \mathrm{E}-00$ & II \\
\hline 251914 & 359.4130 & 0.0909 & 0.03 & 310 & 32.1 & $8.0 \pm 1.3$ & $1.4 \pm 1.2 \mathrm{E}+03$ & $2.7 \pm 4.3 \mathrm{E}-04$ & I \\
\hline 253913 & 359.5316 & 0.1597 & 15.95 & 2 & 15.0 & $5.5 \pm 0.0$ & $1.5 \pm 0.0 \mathrm{E}+02$ & $7.1 \pm 0.0 \mathrm{E}-05$ & I \\
\hline 254708 & 359.0599 & -0.1321 & 0.00 & 595 & 38.6 & $7.7 \pm 1.5$ & $3.0 \pm 2.7 \mathrm{E}+03$ & $0.1 \pm 1.2 \mathrm{E}-04$ & I \\
\hline 255492 & 359.4145 & 0.0845 & 0.00 & 736 & 31.6 & $5.0 \pm 1.6$ & $6.5 \pm 7.8 \mathrm{E}+02$ & $1.1 \pm 3.5 \mathrm{E}-04$ & I \\
\hline 258517 & 359.0967 & -0.1172 & 9.05 & 162 & 15.0 & $7.2 \pm 0.0$ & $1.9 \pm 0.0 \mathrm{E}+03$ & $0.0 \pm 0.0 \mathrm{E}-00$ & III \\
\hline 258882 & 359.2085 & -0.0492 & 4.98 & 23 & 15.0 & $7.4 \pm 0.6$ & $2.1 \pm 0.8 \mathrm{E}+03$ & $0.0 \pm 0.0 \mathrm{E}-00$ & II \\
\hline 262648 & 359.0669 & -0.1440 & 11.83 & 8 & 15.0 & $16.4 \pm 1.0$ & $2.7 \pm 0.4 \mathrm{E}+04$ & $0.0 \pm 0.0 \mathrm{E}-00$ & II \\
\hline 263857 & 359.2346 & -0.0431 & 0.09 & 406 & 38.9 & $8.9 \pm 0.9$ & $4.1 \pm 1.5 \mathrm{E}+03$ & $0.5 \pm 2.7 \mathrm{E}-04$ & $\mathrm{I}$ \\
\hline 264669 & 359.4653 & 0.0970 & 0.00 & 572 & 20.6 & $4.0 \pm 0.6$ & $2.3 \pm 1.5 \mathrm{E}+02$ & $0.2 \pm 3.6 \mathrm{E}-07$ & II \\
\hline 265278 & 359.3205 & 0.0067 & 0.14 & 104 & 16.6 & $4.9 \pm 0.6$ & $2.2 \pm 0.8 \mathrm{E}+02$ & $3.2 \pm 7.4 \mathrm{E}-05$ & I \\
\hline 268132 & 359.1003 & -0.1343 & 3.01 & 11 & 15.0 & $5.5 \pm 0.4$ & $1.7 \pm 0.3 \mathrm{E}+02$ & $4.3 \pm 3.9 \mathrm{E}-04$ & I \\
\hline 268877 & 359.2461 & -0.0461 & 0.03 & 171 & 36.5 & $9.7 \pm 0.9$ & $5.8 \pm 1.8 \mathrm{E}+03$ & $0.8 \pm 1.5 \mathrm{E}-05$ & II \\
\hline 272626 & 359.4288 & 0.0587 & 4.57 & 240 & 18.8 & $10.1 \pm 0.7$ & $6.5 \pm 1.2 \mathrm{E}+03$ & $0.0 \pm 0.0 \mathrm{E}-00$ & II \\
\hline 274996 & 359.2906 & -0.0309 & 1.38 & 4 & 28.0 & $6.8 \pm 0.7$ & $8.0 \pm 1.1 \mathrm{E}+02$ & $7.4 \pm 8.1 \mathrm{E}-04$ & $\mathrm{I}$ \\
\hline 275827 & 359.2791 & -0.0397 & 1.05 & 9 & 15.1 & $8.4 \pm 0.3$ & $1.0 \pm 0.2 \mathrm{E}+03$ & $4.8 \pm 7.0 \mathrm{E}-04$ & I \\
\hline 276597 & 359.4709 & 0.0766 & 5.97 & 52 & 15.0 & $7.5 \pm 0.5$ & $2.3 \pm 0.7 \mathrm{E}+03$ & $0.0 \pm 0.0 \mathrm{E}-00$ & III \\
\hline 277526 & 359.2104 & -0.0854 & 0.25 & 255 & 20.7 & $5.7 \pm 0.7$ & $8.7 \pm 4.0 \mathrm{E}+02$ & $1.5 \pm 9.9 \mathrm{E}-06$ & II \\
\hline 278053 & 359.1057 & -0.1508 & 2.38 & 20 & 45.1 & $9.9 \pm 4.5$ & $6.4 \pm 8.6 \mathrm{E}+03$ & $0.5 \pm 1.5 \mathrm{E}-03$ & $\mathrm{I}$ \\
\hline 279979 & 359.1129 & -0.1501 & 4.59 & 82 & 17.3 & $7.0 \pm 0.6$ & $1.8 \pm 0.5 \mathrm{E}+03$ & $0.0 \pm 0.0 \mathrm{E}-00$ & II \\
\hline 282944 & 359.2387 & -0.0788 & 5.65 & 28 & 15.0 & $4.3 \pm 0.5$ & $1.9 \pm 0.7 \mathrm{E}+02$ & $0.6 \pm 1.6 \mathrm{E}-05$ & I \\
\hline 283981 & 359.2434 & -0.0779 & 3.69 & 42 & 15.0 & $4.3 \pm 0.2$ & $2.9 \pm 0.7 \mathrm{E}+02$ & $0.0 \pm 0.0 \mathrm{E}-00$ & II \\
\hline 284291 & 359.4287 & 0.0353 & 0.07 & 204 & 37.6 & $9.5 \pm 1.0$ & $5.2 \pm 1.9 \mathrm{E}+03$ & $0.8 \pm 3.8 \mathrm{E}-04$ & I \\
\hline 286515 & 359.1828 & -0.1201 & 0.00 & 719 & 32.6 & $7.2 \pm 1.4$ & $2.3 \pm 2.0 \mathrm{E}+03$ & $0.1 \pm 1.0 \mathrm{E}-04$ & I \\
\hline 288646 & 359.2087 & -0.1085 & 4.65 & 15 & 15.0 & $9.6 \pm 2.4$ & $6.4 \pm 4.1 \mathrm{E}+03$ & $0.0 \pm 0.0 \mathrm{E}-00$ & III \\
\hline 293719 & 359.2453 & -0.0959 & 4.27 & 1261 & 15.0 & $6.6 \pm 0.0$ & $1.3 \pm 0.0 \mathrm{E}+03$ & $0.0 \pm 0$. & III \\
\hline 295788 & 359.2963 & -0.0687 & 0.07 & 191 & 35.3 & $7.4 \pm 1.2$ & $1.7 \pm 1.4 \mathrm{E}+03$ & $2.2 \pm 4.4 \mathrm{E}-04$ & I \\
\hline 297164 & 359.4352 & 0.0140 & 0.00 & 739 & 36.2 & $8.4 \pm 0.9$ & $2.4 \pm 1.2 \mathrm{E}+03$ & $1.3 \pm 3.5 \mathrm{E}-04$ & I \\
\hline 297494 & 359.4688 & 0.0340 & 0.00 & 703 & 34.9 & $7.5 \pm 0.9$ & $1.7 \pm 1.0 \mathrm{E}+03$ & $1.7 \pm 4.6 \mathrm{E}-04$ & I \\
\hline 300758 & 359.4562 & 0.0199 & 1.66 & 59 & 17.6 & $8.8 \pm 1.1$ & $3.3 \pm 1.6 \mathrm{E}+03$ & $5.1 \pm 5.2 \mathrm{E}-05$ & I \\
\hline 302651 & 359.3136 & -0.0712 & 0.22 & 318 & 19.6 & $6.6 \pm 0.6$ & $1.4 \pm 0.3 \mathrm{E}+03$ & $0.3 \pm 2.4 \mathrm{E}-04$ & I \\
\hline 302664 & 359.2228 & -0.1270 & 6.28 & 15 & 15.2 & $6.3 \pm 0.7$ & $1.2 \pm 0.4 \mathrm{E}+03$ & $0.0 \pm 0.0 \mathrm{E}-00$ & III \\
\hline 305505 & 359.4583 & 0.0123 & 0.00 & 837 & 38.0 & $10.1 \pm 1.4$ & $5.7 \pm 2.2 \mathrm{E}+03$ & $2.2 \pm 5.1 \mathrm{E}-04$ & I \\
\hline 306363 & 359.5484 & 0.0661 & 0.01 & 441 & 35.1 & $5.7 \pm 1.8$ & $1.2 \pm 1.9 \mathrm{E}+03$ & $0.5 \pm 6.7 \mathrm{E}-05$ & I \\
\hline 307175 & 359.4427 & -0.0002 & 0.00 & 443 & 36.1 & $6.9 \pm 1.0$ & $1.2 \pm 0.9 \mathrm{E}+03$ & $2.2 \pm 5.5 \mathrm{E}-04$ & I \\
\hline 307819 & 359.4249 & -0.0123 & 4.18 & 20 & & $6.2 \pm 0.6$ & $1.1 \pm 0.1 \mathrm{E}+03$ & $1.0 \pm 4.7 \mathrm{E}-04$ & I \\
\hline 311087 & 359.7029 & 0.1526 & 9.19 & 141 & 15 . & $6.4 \pm 0.3$ & $1.2 \pm 0.2 \mathrm{E}+03$ & $0.0 \pm 0.0 \mathrm{E}-00$ & III \\
\hline 311793 & 359.4072 & -0.0300 & 0.00 & 453 & 40.8 & $8.3 \pm 0.8$ & $2.8 \pm 1.2 \mathrm{E}+03$ & $1.0 \pm 3.4 \mathrm{E}-04$ & I \\
\hline 313278 & 359.4882 & 0.0170 & 1.28 & 18 & 16.8 & $6.3 \pm 0.7$ & $2.9 \pm 0.8 \mathrm{E}+02$ & $1.8 \pm 2.3 \mathrm{E}-04$ & $\mathrm{I}$ \\
\hline 314042 & 359.2046 & -0.1584 & 4.66 & 106 & 15.2 & $4.7 \pm 0.5$ & $4.1 \pm 1.7 \mathrm{E}+02$ & $0.0 \pm 0.0 \mathrm{E}-00$ & II \\
\hline 317583 & 359.2173 & -0.1565 & 0.00 & 830 & 30.3 & $4.0 \pm 1.5$ & $1.9 \pm 1.6 \mathrm{E}+02$ & $0.4 \pm 1.3 \mathrm{E}-04$ & $\mathrm{I}$ \\
\hline 317711 & 359.4334 & -0.0241 & 0.08 & 450 & 24.7 & $6.5 \pm 0.8$ & $8.8 \pm 5.9 \mathrm{E}+02$ & $1.0 \pm 2.9 \mathrm{E}-04$ & I \\
\hline 320517 & 359.4441 & -0.0221 & 0.00 & 784 & 39.2 & $9.6 \pm 1.1$ & $5.1 \pm 1.8 \mathrm{E}+03$ & $1.4 \pm 6.3 \mathrm{E}-04$ & I \\
\hline 320729 & 359.3078 & -0.1062 & 7.32 & 14 & 15.0 & $7.1 \pm 0.2$ & $1.8 \pm 0.2 \mathrm{E}+03$ & $0.5 \pm 1.0 \mathrm{E}-06$ & II \\
\hline 321628 & 359.3311 & -0.0934 & 0.33 & 78 & 28.1 & $2.9 \pm 2.4$ & $2.3 \pm 3.3 \mathrm{E}+02$ & $1.4 \pm 3.6 \mathrm{E}-04$ & $\mathrm{I}$ \\
\hline 323541 & 359.5125 & 0.0148 & 5.03 & 55 & 16.6 & $7.0 \pm 1.0$ & $2.0 \pm 0.8 \mathrm{E}+03$ & $0.0 \pm 0.0 \mathrm{E}-00$ & II \\
\hline 328013 & 359.4058 & -0.0580 & 0.00 & 823 & 32.0 & $7.7 \pm 1.6$ & $3.0 \pm 2.8 \mathrm{E}+03$ & $0.1 \pm 1.7 \mathrm{E}-04$ & I \\
\hline 329968 & 359.4136 & -0.0565 & & 73 & & $8.9 \pm 1$ & $4.4 \pm 1.9 \mathrm{E}+03$ & $0.0 \pm 0.0 \mathrm{E}-$ & II \\
\hline 330325 & 359.3839 & -0.0752 & 0.01 & 279 & 37.5 & $8.4 \pm 0.9$ & $2.3 \pm 1.1 \mathrm{E}+03$ & $2.3 \pm 5.8 \mathrm{E}-04$ & I \\
\hline 331261 & 359.2778 & -0.1419 & 6.14 & 48 & 15.3 & $6.0 \pm 0.6$ & $1.0 \pm 0.3 \mathrm{E}+03$ & $0.0 \pm 0.0 \mathrm{E}-00$ & II \\
\hline 331513 & 359.5929 & 0.0510 & 45.54 & 2 & 15.0 & $7.1 \pm 0.0$ & $4.5 \pm 0.0 \mathrm{E}+02$ & $7.6 \pm 0.0 \mathrm{E}-04$ & I \\
\hline 335380 & 359.4371 & -0.0508 & 0.00 & 346 & 30.2 & $9.5 \pm 2.4$ & $5.8 \pm 4.6 \mathrm{E}+03$ & $2.3 \pm 9.5 \mathrm{E}-04$ & $\mathrm{I}$ \\
\hline 336047 & 359.4591 & -0.0384 & 7.85 & 6 & 17.0 & $11.6 \pm 0.8$ & $1.0 \pm 0.2 \mathrm{E}+04$ & $0.0 \pm 0.0 \mathrm{E}-00$ & II \\
\hline 343554 & 359.4277 & -0.0701 & 0.24 & 160 & 35.3 & $7.4 \pm 1.8$ & $2.6 \pm 3.0 \mathrm{E}+03$ & $1.5 \pm 5.3 \mathrm{E}-04$ & $\mathrm{I}$ \\
\hline 344255 & 359.3227 & -0.1357 & 3.44 & 7 & 15.5 & $5.2 \pm 0.5$ & $6.0 \pm 2.2 \mathrm{E}+02$ & $0.0 \pm 0.0 \mathrm{E}-00$ & II \\
\hline 344820 & 359.3390 & -0.1266 & 11.32 & 2 & 15.0 & $6.3 \pm 0.0$ & $2.5 \pm 0.0 \mathrm{E}+02$ & $1.8 \pm 0.0 \mathrm{E}-04$ & I \\
\hline 345503 & 359.5740 & 0.0164 & 1.96 & 140 & 17.7 & $6.6 \pm 0.7$ & $1.5 \pm 0.5 \mathrm{E}+03$ & $0.0 \pm 0.0 \mathrm{E}-00$ & II \\
\hline 346221 & 359.2843 & -0.1625 & 0.53 & 3 & 24.3 & $5.6 \pm 0.6$ & $6.7 \pm 3.2 \mathrm{E}+02$ & $6.5 \pm 6.1 \mathrm{E}-04$ & I \\
\hline 348310 & 359.5823 & 0.0168 & 1.54 & 64 & 16.1 & $5.0 \pm 0.5$ & $5.3 \pm 2.0 \mathrm{E}+02$ & $0.0 \pm 0.0 \mathrm{E}-00$ & II \\
\hline 349188 & 359.5809 & 0.0144 & 0.13 & 195 & 16.5 & $5.6 \pm 0.6$ & $7.9 \pm 2.9 \mathrm{E}+02$ & $0.0 \pm 0.0 \mathrm{E}-00$ & II \\
\hline 351441 & 359.4553 & -0.0662 & 0.03 & 694 & 39.2 & $8.7 \pm 0.9$ & $3.4 \pm 1.4 \mathrm{E}+03$ & $1.2 \pm 4.8 \mathrm{E}-04$ & I \\
\hline
\end{tabular}


Table 3

(Continued)

\begin{tabular}{|c|c|c|c|c|c|c|c|c|c|}
\hline SST GC No. & $l$ & $b$ & $\chi_{\min }^{2}$ & nfits & $\left\langle A_{v}\right\rangle$ & $\left\langle M_{\star}\right\rangle$ & $\left\langle L_{\star}\right\rangle$ & $\left\langle\dot{M}_{\text {env }}\right\rangle$ & $\langle$ Stage $\rangle$ \\
\hline 358063 & 359.5668 & -0.0086 & 2.02 & 33 & 15.1 & $9.5 \pm 1.4$ & $5.7 \pm 2.6 \mathrm{E}+03$ & $0.0 \pm 0.0 \mathrm{E}-00$ & II \\
\hline 359581 & 359.6667 & 0.0501 & 3.94 & 19 & 15.0 & $7.1 \pm 0.3$ & $1.8 \pm 0.3 \mathrm{E}+03$ & $0.0 \pm 0.0 \mathrm{E}-00$ & II \\
\hline 359649 & 359.6652 & 0.0491 & 4.13 & 14 & 15.0 & $6.5 \pm 0.5$ & $1.1 \pm 0.6 \mathrm{E}+03$ & $1.5 \pm 2.9 \mathrm{E}-05$ & I \\
\hline 360104 & 359.4031 & -0.1123 & 1.09 & 35 & 15.3 & $6.6 \pm 0.3$ & $4.1 \pm 1.1 \mathrm{E}+02$ & $2.2 \pm 3.0 \mathrm{E}-04$ & I \\
\hline 360444 & 359.6817 & 0.0578 & 12.18 & 4 & 15.0 & $6.9 \pm 0.1$ & $3.9 \pm 0.5 \mathrm{E}+02$ & $4.1 \pm 3.5 \mathrm{E}-04$ & I \\
\hline 362943 & 359.8239 & 0.1410 & 4.26 & 24 & 15.0 & $4.5 \pm 0.3$ & $2.6 \pm 0.8 \mathrm{E}+02$ & $0.7 \pm 1.7 \mathrm{E}-05$ & I \\
\hline 362952 & 359.5303 & -0.0390 & 3.97 & 6 & 15.6 & $7.1 \pm 0.1$ & $4.7 \pm 0.2 \mathrm{E}+02$ & $2.4 \pm 1.1 \mathrm{E}-05$ & I \\
\hline 363252 & 359.5781 & -0.0101 & 1.55 & 12 & 15.0 & $5.4 \pm 0.3$ & $1.7 \pm 0.2 \mathrm{E}+02$ & $2.9 \pm 3.2 \mathrm{E}-04$ & I \\
\hline 365847 & 359.8251 & 0.1370 & 0.21 & 337 & 32.7 & $9.1 \pm 1.5$ & $5.1 \pm 2.9 \mathrm{E}+03$ & $0.1 \pm 1.7 \mathrm{E}-04$ & I \\
\hline 366204 & 359.6598 & 0.0350 & 6.06 & 72 & 15.7 & $8.0 \pm 1.3$ & $3.3 \pm 1.7 \mathrm{E}+03$ & $0.0 \pm 0.0 \mathrm{E}-00$ & II \\
\hline 370438 & 359.5058 & -0.0663 & 0.22 & 372 & 37.5 & $7.8 \pm 0.9$ & $1.9 \pm 1.0 \mathrm{E}+03$ & $1.4 \pm 3.9 \mathrm{E}-04$ & $\mathrm{I}$ \\
\hline 370894 & 359.6155 & 0.0001 & 1.21 & 3 & 15.0 & $6.5 \pm 0.1$ & $2.5 \pm 0.1 \mathrm{E}+02$ & $1.9 \pm 1.0 \mathrm{E}-04$ & I \\
\hline 371987 & 359.8203 & 0.1238 & 2.36 & 32 & 15.3 & $5.8 \pm 0.5$ & $8.9 \pm 3.0 \mathrm{E}+02$ & $0.0 \pm 0.0 \mathrm{E}-00$ & II \\
\hline 372254 & 359.4249 & -0.1189 & 8.40 & 2 & 16.8 & $6.8 \pm 0.0$ & $3.4 \pm 0.0 \mathrm{E}+02$ & $6.0 \pm 0.0 \mathrm{E}-05$ & $\mathrm{I}$ \\
\hline 372630 & 359.5113 & -0.0666 & 0.06 & 237 & 31.7 & $8.8 \pm 2.8$ & $4.6 \pm 5.0 \mathrm{E}+03$ & $2.2 \pm 7.8 \mathrm{E}-04$ & I \\
\hline 373058 & 359.5164 & -0.0641 & 0.03 & 99 & 31.3 & $7.9 \pm 0.6$ & $2.2 \pm 1.2 \mathrm{E}+03$ & $1.9 \pm 5.9 \mathrm{E}-04$ & I \\
\hline 374813 & 359.5477 & -0.0478 & 0.03 & 1000 & 38.8 & $9.5 \pm 1.0$ & $4.8 \pm 1.8 \mathrm{E}+03$ & $1.0 \pm 4.4 \mathrm{E}-04$ & I \\
\hline 376861 & 359.6040 & -0.0167 & 7.40 & 71 & 15.5 & $7.8 \pm 1.1$ & $2.9 \pm 1.3 \mathrm{E}+03$ & $0.0 \pm 0.0 \mathrm{E}-00$ & II \\
\hline 377183 & 359.3960 & -0.1447 & 5.22 & 31 & 16.2 & $7.8 \pm 0.5$ & $2.7 \pm 0.6 \mathrm{E}+03$ & $0.0 \pm 0.0 \mathrm{E}-00$ & II \\
\hline 380282 & 359.7906 & 0.0920 & 2.73 & 70 & 15.0 & $6.8 \pm 0.9$ & $1.6 \pm 0.8 \mathrm{E}+03$ & $1.0 \pm 7.7 \mathrm{E}-06$ & II \\
\hline 381931 & 359.4557 & -0.1158 & 0.50 & 12 & 27.3 & $3.6 \pm 3.6$ & $7.2 \pm 8.5 \mathrm{E}+02$ & $4.5 \pm 6.9 \mathrm{E}-04$ & I \\
\hline 384976 & 359.7965 & 0.0881 & 8.82 & 45 & 15.0 & $10.4 \pm 0.2$ & $7.2 \pm 0.4 \mathrm{E}+03$ & $0.0 \pm 0.0 \mathrm{E}-00$ & III \\
\hline 386185 & 359.4817 & -0.1068 & 3.58 & 79 & 15.5 & $7.4 \pm 0.5$ & $2.1 \pm 0.6 \mathrm{E}+03$ & $0.0 \pm 0.0 \mathrm{E}-00$ & II \\
\hline 387011 & 359.4816 & -0.1082 & 2.54 & 22 & 15.0 & $5.9 \pm 0.7$ & $1.0 \pm 0.3 \mathrm{E}+03$ & $2.7 \pm 4.0 \mathrm{E}-07$ & II \\
\hline 387018 & 359.7330 & 0.0458 & 8.15 & 41 & 15.0 & $10.2 \pm 0.3$ & $6.7 \pm 0.8 \mathrm{E}+03$ & $0.0 \pm 0.0 \mathrm{E}-00$ & III \\
\hline 390425 & 359.4830 & -0.1129 & 2.85 & 47 & 15.0 & $5.9 \pm 0.9$ & $7.3 \pm 8.8 \mathrm{E}+02$ & $0.5 \pm 1.9 \mathrm{E}-04$ & I \\
\hline 394544 & 359.9475 & 0.1649 & 0.72 & 552 & 27.5 & $5.7 \pm 1.0$ & $9.2 \pm 6.1 \mathrm{E}+02$ & $0.4 \pm 5.4 \mathrm{E}-05$ & II \\
\hline 395315 & 359.5677 & -0.0690 & 0.00 & 1400 & 34.7 & $8.7 \pm 0.9$ & $3.5 \pm 1.3 \mathrm{E}+03$ & $1.1 \pm 4.4 \mathrm{E}-04$ & I \\
\hline 397703 & 359.9313 & 0.1497 & 0.00 & 526 & 28.8 & $7.7 \pm 1.8$ & $2.7 \pm 2.7 \mathrm{E}+03$ & $1.6 \pm 6.7 \mathrm{E}-04$ & I \\
\hline 399231 & 359.9483 & 0.1576 & 0.07 & 528 & 35.1 & $8.0 \pm 1.8$ & $3.5 \pm 4.2 \mathrm{E}+03$ & $0.1 \pm 1.2 \mathrm{E}-04$ & I \\
\hline 399927 & 359.5769 & -0.0709 & 6.83 & 8 & 15.0 & $8.2 \pm 0.5$ & $3.2 \pm 0.7 \mathrm{E}+03$ & $1.5 \pm 2.6 \mathrm{E}-09$ & II \\
\hline 400062 & 359.5126 & -0.1105 & 0.00 & 379 & 33.1 & $6.5 \pm 1.9$ & $1.7 \pm 2.2 \mathrm{E}+03$ & $1.3 \pm 4.1 \mathrm{E}-04$ & I \\
\hline 401264 & 359.5329 & -0.1000 & 0.06 & 281 & 36.0 & $8.8 \pm 1.0$ & $3.3 \pm 1.3 \mathrm{E}+03$ & $1.9 \pm 6.3 \mathrm{E}-04$ & I \\
\hline 409238 & 359.6113 & -0.0651 & 8.31 & 7 & 15.0 & $8.7 \pm 0.0$ & $4.0 \pm 0.0 \mathrm{E}+03$ & $0.0 \pm 0.0 \mathrm{E}-00$ & II \\
\hline 409373 & 359.5185 & -0.1221 & 2.50 & 88 & 17.8 & $6.5 \pm 1.0$ & $1.6 \pm 0.6 \mathrm{E}+03$ & $0.0 \pm 0.0 \mathrm{E}-00$ & II \\
\hline 411192 & 359.6193 & -0.0633 & 0.07 & 303 & 32.6 & $7.0 \pm 2.0$ & $2.4 \pm 2.6 \mathrm{E}+03$ & $0.5 \pm 2.0 \mathrm{E}-04$ & I \\
\hline 412509 & 359.7154 & -0.0066 & 0.09 & 136 & 30.8 & $6.8 \pm 1.1$ & $1.2 \pm 1.3 \mathrm{E}+03$ & $1.5 \pm 4.0 \mathrm{E}-04$ & $\mathrm{I}$ \\
\hline 416141 & 359.9919 & 0.1567 & 15.72 & 13 & 15.0 & $10.7 \pm 1.3$ & $8.2 \pm 3.3 \mathrm{E}+03$ & $0.0 \pm 0.0 \mathrm{E}-00$ & II \\
\hline 417301 & 359.8509 & 0.0685 & 0.60 & 293 & 29.1 & $6.2 \pm 1.5$ & $1.4 \pm 1.5 \mathrm{E}+03$ & $1.0 \pm 7.0 \mathrm{E}-08$ & II \\
\hline 419271 & 359.5965 & -0.0903 & 0.00 & 1079 & 39.6 & $9.0 \pm 1.0$ & $3.7 \pm 1.4 \mathrm{E}+03$ & $1.1 \pm 4.3 \mathrm{E}-04$ & I \\
\hline 420459 & 359.6870 & -0.0368 & 12.33 & 26 & & $13.1 \pm 0.9$ & $1.4 \pm 0.2 \mathrm{E}+04$ & $0.0 \pm 0.0 \mathrm{E}-00$ & II \\
\hline 421092 & 359.6650 & -0.0513 & 0.02 & 417 & 32. & $6.9 \pm 1.0$ & $1.3 \pm 1.1 \mathrm{E}+03$ & $1.6 \pm 4.4 \mathrm{E}-04$ & I \\
\hline 421780 & 359.5573 & -0.1183 & 0.00 & 649 & 33.0 & $5.6 \pm 1.9$ & $1.1 \pm 1.5 \mathrm{E}+03$ & $0.9 \pm 3.6 \mathrm{E}-04$ & I \\
\hline 431993 & 359.7828 & 0.0031 & 6.64 & 7 & 15.0 & $7.0 \pm 0.0$ & $4.8 \pm 0.2 \mathrm{E}+02$ & $2.7 \pm 3.2 \mathrm{E}-04$ & I \\
\hline 436449 & 359.6986 & -0.0554 & 5.46 & 673 & 15.0 & $9.9 \pm 0.3$ & $6.1 \pm 0.8 \mathrm{E}+03$ & $0.0 \pm 0.0 \mathrm{E}-00$ & III \\
\hline 440627 & 359.8361 & 0.0218 & 2.03 & 2 & 15.0 & $7.9 \pm 0.0$ & $7.5 \pm 0.0 \mathrm{E}+02$ & $4.9 \pm 3.3 \mathrm{E}-04$ & I \\
\hline 441299 & 359.7169 & -0.0521 & 6.74 & 53 & 15.0 & $8.5 \pm 0.7$ & $3.8 \pm 1.1 \mathrm{E}+03$ & $0.0 \pm 0.0 \mathrm{E}-00$ & II \\
\hline 442291 & 359.7696 & -0.0215 & 5.83 & 5 & 15.0 & $7.2 \pm 0.2$ & $1.9 \pm 0.2 \mathrm{E}+03$ & $0.6 \pm 1.1 \mathrm{E}-07$ & II \\
\hline 442669 & 359.6671 & -0.0848 & 11.06 & 5 & 15.0 & $10.3 \pm 0.1$ & $6.8 \pm 0.3 \mathrm{E}+03$ & $0.0 \pm 0.0 \mathrm{E}-00$ & II \\
\hline 447593 & 359.8057 & -0.0080 & 4.63 & 6 & 15.0 & $8.5 \pm 0.0$ & $3.6 \pm 0.0 \mathrm{E}+03$ & $0.0 \pm 0.0 \mathrm{E}-00$ & II \\
\hline 448031 & 359.8111 & -0.0054 & 12.85 & 10 & 15.0 & $8.3 \pm 0.7$ & $2.9 \pm 1.5 \mathrm{E}+03$ & $1.8 \pm 4.6 \mathrm{E}-05$ & I \\
\hline 454105 & 359.7053 & -0.0798 & 0.00 & 332 & 34.0 & $8.4 \pm 2.3$ & $4.5 \pm 5.9 \mathrm{E}+03$ & $0.1 \pm 1.9 \mathrm{E}-04$ & I \\
\hline 454135 & & -0.1414 & & 32 & 33. & $5.9 \pm 1$ & $1.2 \pm 1.7 \mathrm{E}+03$ & $1.7 \pm 4.9 \mathrm{E}-$ & I \\
\hline 457947 & 359.6273 & -0.1336 & 0.08 & 736 & 31.3 & $6.5 \pm 0.9$ & $1.0 \pm 0.6 \mathrm{E}+03$ & $0.5 \pm 2.0 \mathrm{E}-04$ & I \\
\hline 458543 & 359.7303 & -0.0716 & 0.19 & 102 & 31.2 & $7.3 \pm 0.9$ & $2.0 \pm 1.0 \mathrm{E}+03$ & $0.7 \pm 3.6 \mathrm{E}-04$ & I \\
\hline 460967 & 359.7678 & -0.0526 & 7.90 & 4 & 15.0 & $7.3 \pm 0.0$ & $2.0 \pm 0.0 \mathrm{E}+03$ & $6.1 \pm 0.0 \mathrm{E}-09$ & II \\
\hline 462136 & 359.6277 & -0.1401 & 1.96 & 66 & 15.3 & $6.3 \pm 1.4$ & $1.4 \pm 1.6 \mathrm{E}+03$ & $0.4 \pm 1.5 \mathrm{E}-05$ & II \\
\hline 462303 & 359.6423 & -0.1314 & 4.23 & 23 & 15.0 & $5.7 \pm 0.2$ & $2.3 \pm 0.5 \mathrm{E}+02$ & $1.1 \pm 3.3 \mathrm{E}-04$ & I \\
\hline 464434 & 359.6721 & -0.1166 & 8.13 & 10 & 15.0 & $7.8 \pm 0.7$ & $2.7 \pm 1.0 \mathrm{E}+03$ & $0.0 \pm 0.0 \mathrm{E}-00$ & III \\
\hline 466603 & 359.8973 & 0.0175 & 2.58 & 36 & 15.5 & $6.9 \pm 0.6$ & $9.7 \pm 3.2 \mathrm{E}+02$ & $0.8 \pm 1.6 \mathrm{E}-04$ & I \\
\hline 470861 & 359.8013 & -0.0478 & 11.32 & 9 & 16.0 & $7.7 \pm 0.4$ & $2.4 \pm 0.5 \mathrm{E}+03$ & $3.4 \pm 3.0 \mathrm{E}-09$ & II \\
\hline 472034 & 359.7097 & -0.1056 & 4.29 & 43 & 15.3 & $8.1 \pm 0.8$ & $3.1 \pm 1.1 \mathrm{E}+03$ & $0.0 \pm 0.0 \mathrm{E}-00$ & II \\
\hline 474477 & 359.8252 & -0.0389 & 0.00 & 393 & 31.0 & $5.4 \pm 1.6$ & $0.7 \pm 1.1 \mathrm{E}+03$ & $1.6 \pm 4.5 \mathrm{E}-04$ & I \\
\hline 475651 & 359.7068 & -0.1132 & 5.01 & 46 & 15.0 & $9.5 \pm 0.6$ & $5.3 \pm 1.2 \mathrm{E}+03$ & $0.0 \pm 0.0 \mathrm{E}-00$ & III \\
\hline 476516 & 359.7604 & -0.0818 & 0.00 & 334 & 31.3 & $8.6 \pm 2.2$ & $4.5 \pm 4.2 \mathrm{E}+03$ & $1.2 \pm 5.6 \mathrm{E}-04$ & I \\
\hline 479032 & 359.6584 & -0.1481 & 1.39 & 68 & 16.1 & $6.8 \pm 1.0$ & $1.6 \pm 1.0 \mathrm{E}+03$ & $0.3 \pm 2.6 \mathrm{E}-04$ & I \\
\hline
\end{tabular}


Table 3

(Continued)

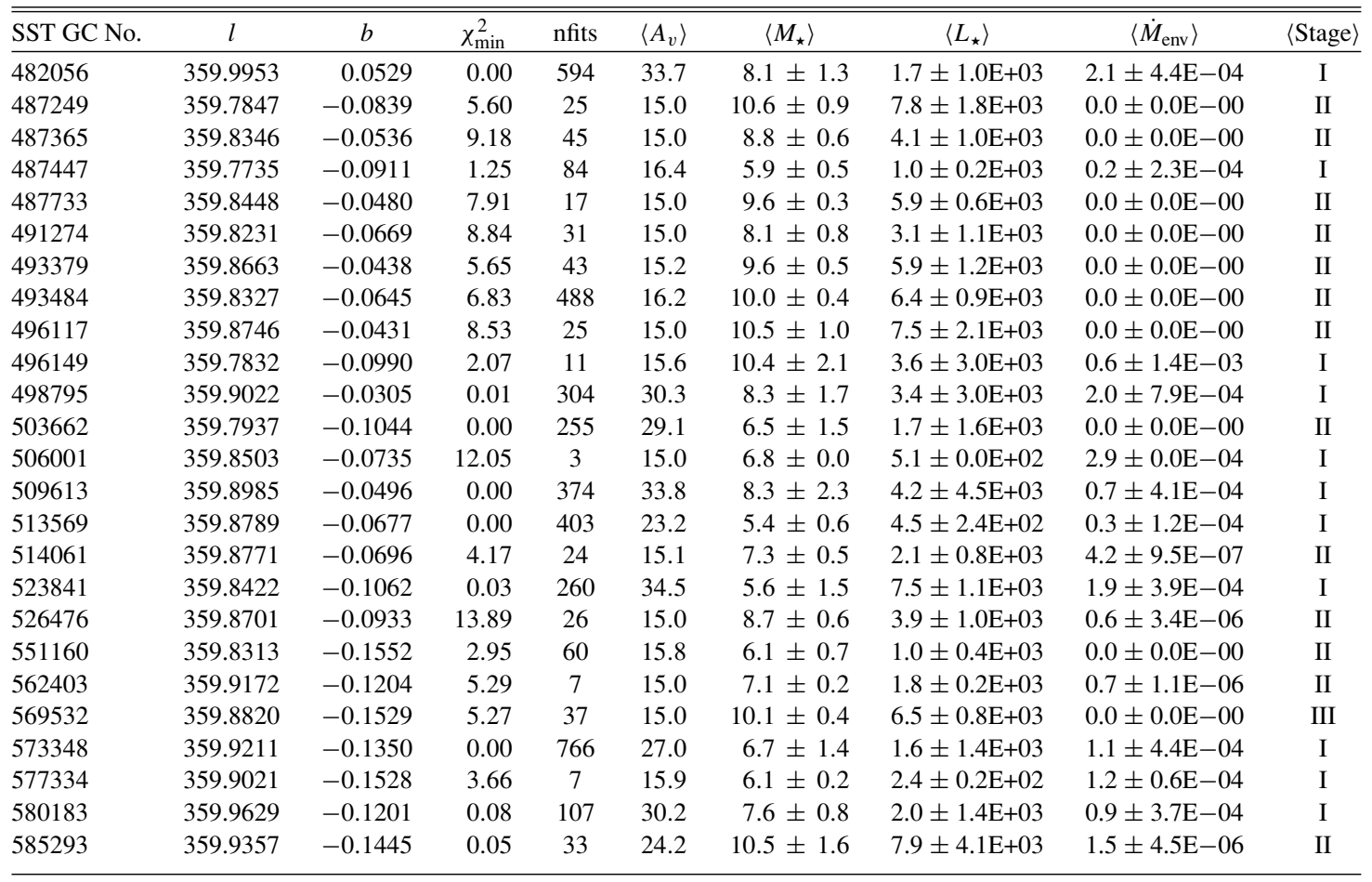

the G359.43+0.02 cluster. The total fraction of the SED fitted YSOs in Stage I is $\sim 60 \%$. Assuming that these sources are within the central region and hence located at distances of 7.5 and $8.5 \mathrm{kpc}$, Tables 3 and 4 list the physical parameters of individual sources distributed at negative and positive longitudes, respectively.

\subsection{Star Formation Rate \\ 5.3.1. G359.43+0.02 Cluster}

We now estimate the SFR from the sum of average masses and luminosities derived from fitting of the SEDs of the YSOs. We first determine the SFR in the G359.43+0.02 cluster which is a subset of the detected YSOs found throughout the nuclear disk. The characteristics of this cluster are described in Section 5.1. The total stellar mass in the cluster is estimated to be $\sim 148 M_{\odot}$. Assuming a distance of $\sim 8.5 \mathrm{kpc}$, SED fitting to a sample of 18 sources yields masses ranging from 8 to $20 M_{\odot}$ and classifies 14 candidates in Stage I, 3 in Stage II, and 1 in Stage III. This result suggests that the majority (nominally $78 \%$ ) are Stage I YSOs, which have stellar ages $\sim 10^{5} \mathrm{yr}$ and masses in excess of $8 M_{\odot}$ or low-mass stars. The canonical age for a Class I source for low-mass stars is thought to be $(1-5) \times 10^{5} \mathrm{yr}$. This is based on the ratio of number of Class I to Class II sources in nearby star-forming regions. The ages of the Class II sources are known from their stellar parameters (temperature, radius) and PMS stellar evolutionary tracks, so a combination of theory and observations and Class 0 sources are estimated to be $\sim 10^{4} \mathrm{yr}$, from ratio of the numbers and dynamical timescales of their outflows. For higher mass stars which presumably form much faster, we believe $10^{5} \mathrm{yr}$ for a Stage I source is reasonable (Lada 1999; Beuther et al. 2007).

To compute the total cluster mass of only Stage I YSOs, we adopt a standard broken power-law form of the initial mass function (IMF; Whitney et al. 2008; Kroupa 2001), with the number of stars per unit mass scaling as $M^{-1.3}$ between 0.08 and
$0.5 M_{\odot}$ and as $M^{-2.3}$ between $0.5 M_{\odot}$ and $50 M_{\odot}$ (designated the Kroupa IMF hereafter). Figure 12(b) shows the broken power-law form, as dashed line, which is normalized to pass through the peak in the histogram of the stellar mass distribution derived from SED fitting of the YSOs in the G359.43+0.02 cluster. The stellar mass associated with the Stage I YSOs in this cluster is $\sim 1400 M_{\odot}$.

The lack of the observed high-mass stars is most likely due to saturated sources and confusion. Another possibility is that the SED models of massive stars with a large inner hole in the surrounding material were not included in the grid, and thus are not represented in this study. Lastly, most massive stars embedded in the dense molecular material, which is on average $10^{2}$ higher than that in the Galactic disk, may evolve rapidly. The residence time of an expanding remnant in dense molecular clouds such as found in the Galactic center is unknown. Numerical simulations are needed to examine whether the evolution of supernova remnants (SNR) give shorter residence time in high density medium than in low density medium (Tilley et al. 2006). This may be relevant to the fact that the Galactic center region is known to host a high cosmic ray flux (Law et al. 2008). The advantage of using only Stage I YSOs is that the average lifetime of these sources is better constrained than when all YSOs are included. Assuming that the typical age of Stage I YSOs is $10^{5} \mathrm{yr}$, the SFR in this cluster is $\sim 1.4 \times 10^{-2} M_{\odot} \mathrm{yr}^{-1}$.

\subsubsection{Nuclear Disk}

SFR Using YSO Candidates. We now estimate the SFR in the nuclear disk restricted to a region between $|l|<1.3$ and $|b|<10^{\prime}$. The characteristics of the YSOs are described in Section 5.2. The stellar mass associated with only Stage I YSOs gives a total mass of $\sim 1.4 \times 10^{4} M_{\odot}$. The SFR is estimated to be $\sim 0.14 M_{\odot} \mathrm{yr}^{-1}$. Figure 12(c) shows the histogram of the mass distribution for both longitudes as well as the broken 
Table 4

The Parameters of the SED Fits to Candidate YSOs in $l>0^{\circ}$

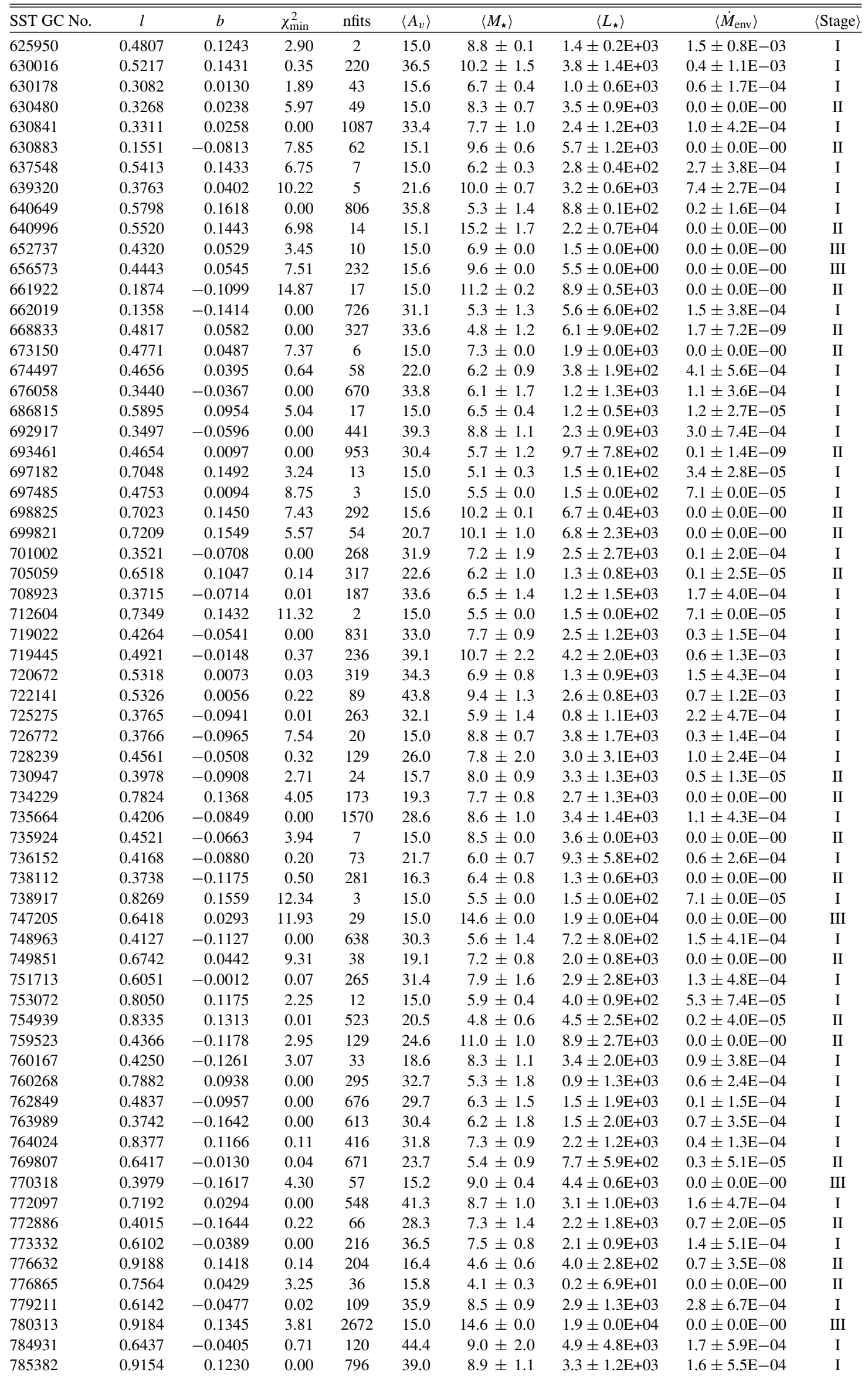


Table 4

(Continued)

\begin{tabular}{|c|c|c|c|c|c|c|c|c|c|}
\hline SST GC No. & $l$ & $b$ & $\chi_{\min }^{2}$ & nfits & $\left\langle A_{v}\right\rangle$ & $\left\langle M_{\star}\right\rangle$ & $\left\langle L_{\star}\right\rangle$ & $\left\langle\dot{M}_{\text {env }}\right\rangle$ & $\langle$ Stage $\rangle$ \\
\hline 787884 & 0.6428 & -0.0466 & 0.01 & 475 & 35.0 & $9.0 \pm 1.2$ & $2.5 \pm 1.1 \mathrm{E}+03$ & $2.8 \pm 7.2 \mathrm{E}-04$ & I \\
\hline 790413 & 0.5867 & -0.0854 & 0.37 & 76 & 29.1 & $8.2 \pm 0.8$ & $1.3 \pm 0.5 \mathrm{E}+03$ & $3.8 \pm 7.3 \mathrm{E}-04$ & I \\
\hline 793536 & 0.6484 & -0.0540 & 47.79 & 1 & 15.0 & $7.9 \pm 0.0$ & $7.4 \pm 0.0 \mathrm{E}+02$ & $1.5 \pm 0.0 \mathrm{E}-04$ & I \\
\hline 793867 & 0.6818 & -0.0344 & 0.14 & 336 & 36.7 & $8.6 \pm 1.0$ & $3.0 \pm 1.7 \mathrm{E}+03$ & $2.0 \pm 6.1 \mathrm{E}-04$ & I \\
\hline 795418 & 0.6355 & -0.0653 & 0.00 & 703 & 31.9 & $6.6 \pm 1.1$ & $1.1 \pm 0.9 \mathrm{E}+03$ & $1.6 \pm 4.3 \mathrm{E}-04$ & I \\
\hline 795658 & 0.5621 & -0.1102 & 0.08 & 150 & 38.6 & $5.7 \pm 1.5$ & $1.1 \pm 1.7 \mathrm{E}+03$ & $1.3 \pm 4.5 \mathrm{E}-04$ & I \\
\hline 795914 & 0.8815 & 0.0826 & 0.39 & 467 & 35.6 & $5.1 \pm 1.0$ & $0.6 \pm 1.1 \mathrm{E}+03$ & $1.3 \pm 8.7 \mathrm{E}-05$ & I \\
\hline 796410 & 0.6606 & -0.0520 & 0.04 & 339 & 32.0 & $7.5 \pm 1.3$ & $2.0 \pm 1.8 \mathrm{E}+03$ & $1.6 \pm 5.0 \mathrm{E}-04$ & I \\
\hline 797252 & 0.6810 & -0.0413 & 0.00 & 405 & 30.1 & $7.3 \pm 2.0$ & $2.6 \pm 3.2 \mathrm{E}+03$ & $1.4 \pm 5.7 \mathrm{E}-04$ & I \\
\hline 801788 & 0.8674 & 0.0629 & 4.41 & 146 & 15.0 & $4.5 \pm 0.0$ & $3.3 \pm 0.0 \mathrm{E}+02$ & $0.0 \pm 0.0 \mathrm{E}-00$ & II \\
\hline 802032 & 0.8693 & 0.0635 & 0.00 & 1040 & 29.9 & $4.4 \pm 0.9$ & $3.7 \pm 3.4 \mathrm{E}+02$ & $0.1 \pm 2.6 \mathrm{E}-07$ & II \\
\hline 802748 & 0.7511 & -0.0093 & 0.12 & 199 & 18.2 & $5.1 \pm 0.6$ & $3.2 \pm 1.1 \mathrm{E}+02$ & $0.4 \pm 1.5 \mathrm{E}-04$ & $\mathrm{I}$ \\
\hline 802877 & 0.8718 & 0.0634 & 2.99 & 14 & 15.0 & $3.8 \pm 0.3$ & $1.7 \pm 0.5 \mathrm{E}+02$ & $0.0 \pm 0.0 \mathrm{E}-00$ & II \\
\hline 804299 & 0.8768 & 0.0636 & 0.04 & 423 & 17.7 & $3.7 \pm 0.4$ & $1.5 \pm 0.8 \mathrm{E}+02$ & $1.3 \pm 4.8 \mathrm{E}-08$ & II \\
\hline 805200 & 0.6179 & -0.0948 & 0.00 & 462 & 37.5 & $8.5 \pm 0.9$ & $3.0 \pm 1.0 \mathrm{E}+03$ & $2.1 \pm 6.9 \mathrm{E}-04$ & $\mathrm{I}$ \\
\hline 806191 & 0.6326 & -0.0880 & 0.02 & 154 & 38.9 & $8.8 \pm 1.3$ & $2.0 \pm 0.9 \mathrm{E}+03$ & $0.6 \pm 1.1 \mathrm{E}-03$ & I \\
\hline 807365 & 0.9825 & 0.1211 & 0.00 & 502 & 29.2 & $6.0 \pm 1.5$ & $1.3 \pm 1.6 \mathrm{E}+03$ & $0.7 \pm 5.8 \mathrm{E}-09$ & II \\
\hline 807789 & 0.5456 & -0.1440 & 0.00 & 510 & 29.4 & $6.3 \pm 1.9$ & $1.6 \pm 2.2 \mathrm{E}+03$ & $0.8 \pm 3.7 \mathrm{E}-04$ & $\mathrm{I}$ \\
\hline 809058 & 0.5288 & -0.1569 & 8.77 & 9 & 15.0 & $7.6 \pm 0.7$ & $2.4 \pm 0.8 \mathrm{E}+03$ & $0.5 \pm 1.0 \mathrm{E}-06$ & II \\
\hline 809602 & 0.9572 & 0.1011 & 1.05 & 306 & 39.1 & $5.3 \pm 1.0$ & $7.6 \pm 6.0 \mathrm{E}+02$ & $0.1 \pm 1.1 \mathrm{E}-04$ & I \\
\hline 810562 & 0.6899 & -0.0626 & 0.00 & 555 & 35.9 & $5.9 \pm 1.7$ & $1.3 \pm 1.8 \mathrm{E}+03$ & $0.4 \pm 2.3 \mathrm{E}-04$ & I \\
\hline 811158 & 0.5871 & -0.1260 & 0.00 & 1127 & 37.9 & $9.4 \pm 1.1$ & $4.5 \pm 1.7 \mathrm{E}+03$ & $1.1 \pm 4.6 \mathrm{E}-04$ & I \\
\hline 811550 & 0.7552 & -0.0252 & 0.02 & 615 & 26.2 & $6.4 \pm 0.7$ & $8.6 \pm 4.3 \mathrm{E}+02$ & $0.7 \pm 2.5 \mathrm{E}-04$ & I \\
\hline 812377 & 0.6003 & -0.1205 & 0.12 & 390 & 40.8 & $8.5 \pm 0.8$ & $2.9 \pm 1.2 \mathrm{E}+03$ & $1.2 \pm 3.6 \mathrm{E}-04$ & I \\
\hline 814299 & 0.7259 & -0.0485 & 0.02 & 1052 & 29.3 & $5.2 \pm 1.1$ & $7.2 \pm 6.2 \mathrm{E}+02$ & $0.3 \pm 9.9 \mathrm{E}-06$ & II \\
\hline 818720 & 0.7520 & -0.0419 & 0.01 & 492 & 30.3 & $6.5 \pm 1.2$ & $1.5 \pm 1.1 \mathrm{E}+03$ & $0.0 \pm 0.0 \mathrm{E}-00$ & II \\
\hline 819806 & 0.5527 & -0.1647 & 0.02 & 807 & 35.0 & $6.5 \pm 1.0$ & $1.3 \pm 1.0 \mathrm{E}+03$ & $0.3 \pm 1.2 \mathrm{E}-04$ & $\mathrm{I}$ \\
\hline 820972 & 0.8029 & -0.0159 & 6.60 & 51 & 15.7 & $6.7 \pm 0.7$ & $1.4 \pm 0.5 \mathrm{E}+03$ & $0.9 \pm 4.4 \mathrm{E}-04$ & I \\
\hline 821060 & 0.5660 & -0.1594 & 0.00 & 706 & 30.6 & $5.4 \pm 1.4$ & $6.7 \pm 7.6 \mathrm{E}+02$ & $1.3 \pm 3.9 \mathrm{E}-04$ & I \\
\hline 826501 & 0.7728 & -0.0458 & 8.19 & 10 & 17.0 & $5.9 \pm 0.6$ & $2.2 \pm 0.3 \mathrm{E}+02$ & $4.0 \pm 3.6 \mathrm{E}-04$ & I \\
\hline 826931 & 0.9561 & 0.0640 & 3.85 & 69 & 15.5 & $5.2 \pm 0.4$ & $6.1 \pm 1.8 \mathrm{E}+02$ & $0.0 \pm 0.0 \mathrm{E}-00$ & II \\
\hline 828786 & 0.7263 & -0.0789 & 0.06 & 520 & 27.2 & $4.4 \pm 0.8$ & $3.6 \pm 2.4 \mathrm{E}+02$ & $0.0 \pm 1.4 \mathrm{E}-05$ & II \\
\hline 829672 & 1.0693 & 0.1266 & 0.00 & 985 & 27.2 & $4.3 \pm 0.7$ & $3.1 \pm 2.5 \mathrm{E}+02$ & $0.1 \pm 2.0 \mathrm{E}-05$ & II \\
\hline 830399 & 0.5877 & -0.1661 & 0.38 & 172 & 16.8 & $4.1 \pm 0.8$ & $1.2 \pm 1.0 \mathrm{E}+02$ & $2.5 \pm 6.6 \mathrm{E}-05$ & I \\
\hline 830971 & 0.9619 & 0.0588 & 0.00 & 838 & 27.6 & $5.0 \pm 0.8$ & $5.5 \pm 3.9 \mathrm{E}+02$ & $0.1 \pm 3.3 \mathrm{E}-05$ & II \\
\hline 831103 & 1.0790 & 0.1294 & 7.16 & 14 & 15.0 & $6.4 \pm 0.6$ & $1.0 \pm 0.6 \mathrm{E}+03$ & $1.5 \pm 2.9 \mathrm{E}-05$ & I \\
\hline 831236 & 0.5899 & -0.1665 & 9.66 & 1 & 15.0 & $6.0 \pm 0.0$ & $2.1 \pm 0.0 \mathrm{E}+02$ & $9.8 \pm 0.0 \mathrm{E}-05$ & I \\
\hline 837363 & 0.6703 & -0.1307 & 0.13 & 378 & & $4.8 \pm 1.0$ & $1.7 \pm 1.0 \mathrm{E}+02$ & $8.6 \pm 1.8 \mathrm{E}-04$ & I \\
\hline 839447 & 0.6195 & -0.1657 & 0.01 & 635 & 41.8 & $11.1 \pm 1.4$ & $8.0 \pm 2.6 \mathrm{E}+03$ & $1.7 \pm 6.5 \mathrm{E}-04$ & I \\
\hline 841071 & 0.8188 & -0.0487 & 0.02 & 438 & 35.2 & $6.7 \pm 1.1$ & $1.2 \pm 1.0 \mathrm{E}+03$ & $1.3 \pm 3.4 \mathrm{E}-04$ & I \\
\hline 842086 & 0.7590 & -0.0869 & 6.51 & 85 & 22.8 & $9.8 \pm 0.8$ & $6.1 \pm 1.5 \mathrm{E}+03$ & $0.0 \pm 0.0 \mathrm{E}-00$ & II \\
\hline 844666 & 0.8868 & -0.0152 & 8.10 & 9 & 46.8 & $9.8 \pm 1.9$ & $6.4 \pm 3.8 \mathrm{E}+03$ & $0.0 \pm 0.0 \mathrm{E}-00$ & II \\
\hline 848731 & 0.9049 & -0.0128 & 2.85 & 11 & 15.0 & $3.9 \pm 0.2$ & $1.2 \pm 0.4 \mathrm{E}+02$ & $1.5 \pm 1.1 \mathrm{E}-08$ & II \\
\hline 849203 & 0.9969 & 0.0417 & 10.44 & 19 & 15.0 & $7.9 \pm 0.5$ & $2.8 \pm 0.7 \mathrm{E}+03$ & $0.0 \pm 0.0 \mathrm{E}-00$ & III \\
\hline 849731 & 0.9969 & 0.0405 & 22.12 & 3 & 15.0 & $6.6 \pm 0.0$ & $4.6 \pm 0.0 \mathrm{E}+02$ & $1.7 \pm 0.0 \mathrm{E}-05$ & I \\
\hline 850592 & 0.8439 & -0.0537 & 0.04 & 162 & 33.8 & $9.2 \pm 1.0$ & $4.6 \pm 1.6 \mathrm{E}+03$ & $1.3 \pm 7.5 \mathrm{E}-04$ & I \\
\hline 853347 & 1.0547 & 0.0677 & 4.64 & 42 & 15.0 & $9.2 \pm 0.2$ & $4.7 \pm 0.4 \mathrm{E}+03$ & $0.0 \pm 0.0 \mathrm{E}-00$ & III \\
\hline 857133 & 1.0477 & 0.0553 & 0.00 & 213 & 34.1 & $7.3 \pm 0.7$ & $1.8 \pm 0.9 \mathrm{E}+03$ & $0.5 \pm 2.7 \mathrm{E}-04$ & I \\
\hline 857708 & 0.8444 & -0.0687 & 4.52 & 5 & 15.0 & $6.0 \pm 0.3$ & $2.4 \pm 0.4 \mathrm{E}+02$ & $0.1 \pm 7.3 \mathrm{E}-05$ & I \\
\hline 860855 & 0.8333 & -0.0820 & 2.64 & 10 & 15.0 & $6.9 \pm 0.0$ & $1.5 \pm 0.0 \mathrm{E}+03$ & $0.0 \pm 0.0 \mathrm{E}-00$ & III \\
\hline 861689 & 0.9902 & 0.0108 & 0.62 & 332 & 32.1 & $8.0 \pm 1.2$ & $3.1 \pm 2.1 \mathrm{E}+03$ & $0.6 \pm 8.6 \mathrm{E}-05$ & II \\
\hline 864818 & 0.8977 & -0.0516 & 4.37 & 25 & 43.9 & $11.2 \pm 0.8$ & $9.6 \pm 1.9 \mathrm{E}+03$ & $0.0 \pm 0.0 \mathrm{E}-00$ & II \\
\hline 866280 & 0.8145 & -0.1051 & 5.90 & 1156 & 15.0 & $6.6 \pm 0.1$ & $1.3 \pm 0.1 \mathrm{E}+03$ & $0.0 \pm 0.0 \mathrm{E}-00$ & III \\
\hline 868530 & 0.7986 & -0.1198 & 1.88 & 65 & 15.0 & $6.0 \pm 0.5$ & $1.0 \pm 0.3 \mathrm{E}+03$ & $0.0 \pm 0.0 \mathrm{E}-00$ & III \\
\hline 869198 & 1.0000 & 0.0001 & 5.10 & 47 & 15.2 & $4.1 \pm 0.4$ & $2.4 \pm 0.8 \mathrm{E}+02$ & $1.0 \pm 6.7 \mathrm{E}-06$ & II \\
\hline 869219 & 0.8074 & -0.1161 & 6.28 & 3 & 15.0 & $5.1 \pm 0.0$ & $1.5 \pm 0.0 \mathrm{E}+02$ & $4.6 \pm 0.0 \mathrm{E}-05$ & I \\
\hline 869244 & 0.9121 & -0.0530 & 3.99 & 72 & 42.1 & $9.6 \pm 0.0$ & $5.5 \pm 0.0 \mathrm{E}+03$ & $0.0 \pm 0.0 \mathrm{E}-00$ & III \\
\hline 869472 & 0.9372 & -0.0384 & 0.03 & 426 & 28.4 & $3.7 \pm 0.8$ & $1.3 \pm 1.2 \mathrm{E}+02$ & $0.3 \pm 1.8 \mathrm{E}-04$ & I \\
\hline 871019 & 0.7332 & -0.1652 & 6.19 & 735 & 15.0 & $10.0 \pm 0.3$ & $6.2 \pm 0.6 \mathrm{E}+03$ & $0.0 \pm 0.0 \mathrm{E}-00$ & III \\
\hline 878691 & 0.8826 & -0.0935 & 0.69 & 5 & 16.5 & & $1.0 \pm 0.0 \mathrm{E}+03$ & $3.4 \pm 5.5 \mathrm{E}-04$ & I \\
\hline 879350 & 1.0082 & -0.0193 & 5.28 & 24 & 16.0 & $5.9 \pm 0.5$ & $9.4 \pm 3.2 \mathrm{E}+02$ & $0.0 \pm 0.0 \mathrm{E}-00$ & II \\
\hline 879793 & 0.7827 & -0.1564 & 2.37 & 16 & 15.0 & $7.3 \pm 0.2$ & $1.9 \pm 0.2 \mathrm{E}+03$ & $1.6 \pm 0.4 \mathrm{E}-08$ & II \\
\hline 881125 & 0.7777 & -0.1626 & 11.09 & 17 & 15.0 & $6.8 \pm 0.4$ & $1.4 \pm 0.5 \mathrm{E}+03$ & $2.1 \pm 5.7 \mathrm{E}-05$ & I \\
\hline 884709 & 0.9500 & -0.0671 & 0.05 & 1169 & 28.5 & $3.5 \pm 0.7$ & $1.5 \pm 1.4 \mathrm{E}+02$ & $0.3 \pm 8.0 \mathrm{E}-06$ & II \\
\hline 884809 & 1.0370 & -0.0149 & 4.32 & 7 & 15.0 & $5.5 \pm 0.0$ & $1.7 \pm 0.1 \mathrm{E}+02$ & $5.4 \pm 1.4 \mathrm{E}-05$ & $\mathrm{I}$ \\
\hline 887636 & 0.9713 & -0.0612 & 3.59 & 9 & 15.3 & $5.9 \pm 0.6$ & $2.5 \pm 0.3 \mathrm{E}+02$ & $2.5 \pm 1.9 \mathrm{E}-04$ & I \\
\hline
\end{tabular}


Table 4

(Continued)

\begin{tabular}{lccccccccc}
\hline \hline SST GC No. & $l$ & $b$ & $\chi_{\min }^{2}$ & nfits & $\left\langle A_{v}\right\rangle$ & $\left\langle M_{\star}\right\rangle$ & $\left\langle L_{\star}\right\rangle$ & $\left\langle\dot{M}_{\text {env }}\right\rangle$ & $\langle$ Stage $\rangle$ \\
\hline 891214 & 1.1138 & 0.0161 & 0.01 & 350 & 28.5 & $4.3 \pm 1.8$ & $4.4 \pm 6.3 \mathrm{E}+02$ & $1.2 \pm 2.6 \mathrm{E}-04$ & $\mathrm{I}$ \\
891918 & 1.0501 & -0.0239 & 5.60 & 74 & 18.9 & $7.5 \pm 0.5$ & $2.3 \pm 0.6 \mathrm{E}+03$ & $0.0 \pm 0.0 \mathrm{E}-00$ & $\mathrm{II}$ \\
892828 & 0.8474 & -0.1484 & 6.14 & 15 & 15.0 & $5.4 \pm 0.2$ & $5.0 \pm 2.6 \mathrm{E}+02$ & $1.7 \pm 2.5 \mathrm{E}-05$ & $\mathrm{I}$ \\
899543 & 1.1011 & -0.0113 & 0.06 & 225 & 29.0 & $6.9 \pm 1.9$ & $2.3 \pm 3.0 \mathrm{E}+03$ & $0.9 \pm 4.0 \mathrm{E}-04$ & $\mathrm{I}$ \\
899611 & 0.9782 & -0.0856 & 14.19 & 2 & 15.0 & $5.5 \pm 0.0$ & $1.5 \pm 0.0 \mathrm{E}+02$ & $7.1 \pm 0.0 \mathrm{E}-05$ & $\mathrm{I}$ \\
\hline
\end{tabular}
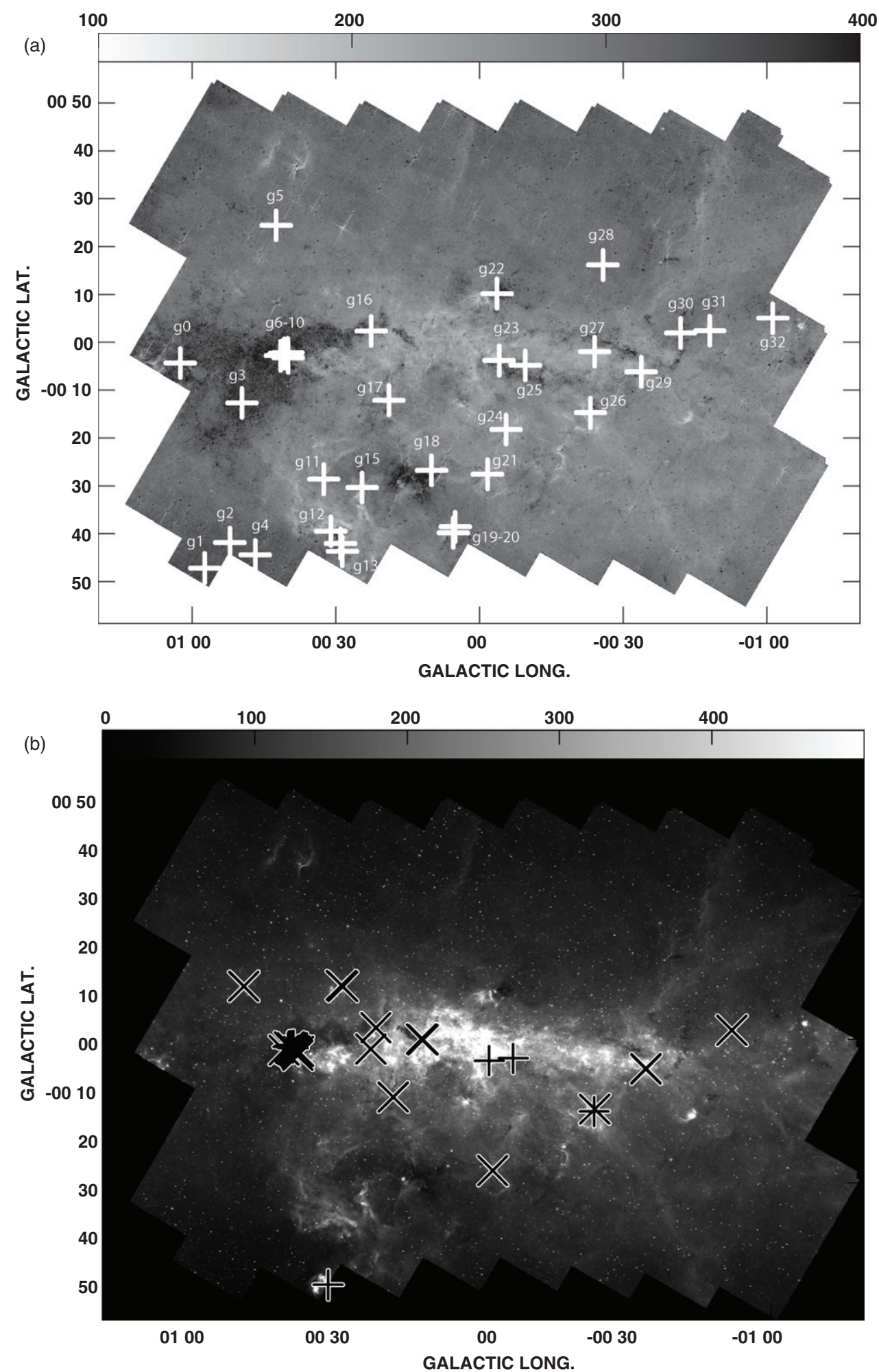

Figure 14. (a) Distribution of $4.5 \mu \mathrm{m}$ excess sources, presented as crosses, is superimposed on a ratio image constructed from $I(4.5) /\left[I(3.6)^{1.2} \times I(5.8)\right]^{0.5}$. (b) The distribution of class I and II methanol masers, shown as "+" and " $\times$ " signs, respectively is superimposed on an $8 \mu \mathrm{m}$ image. 

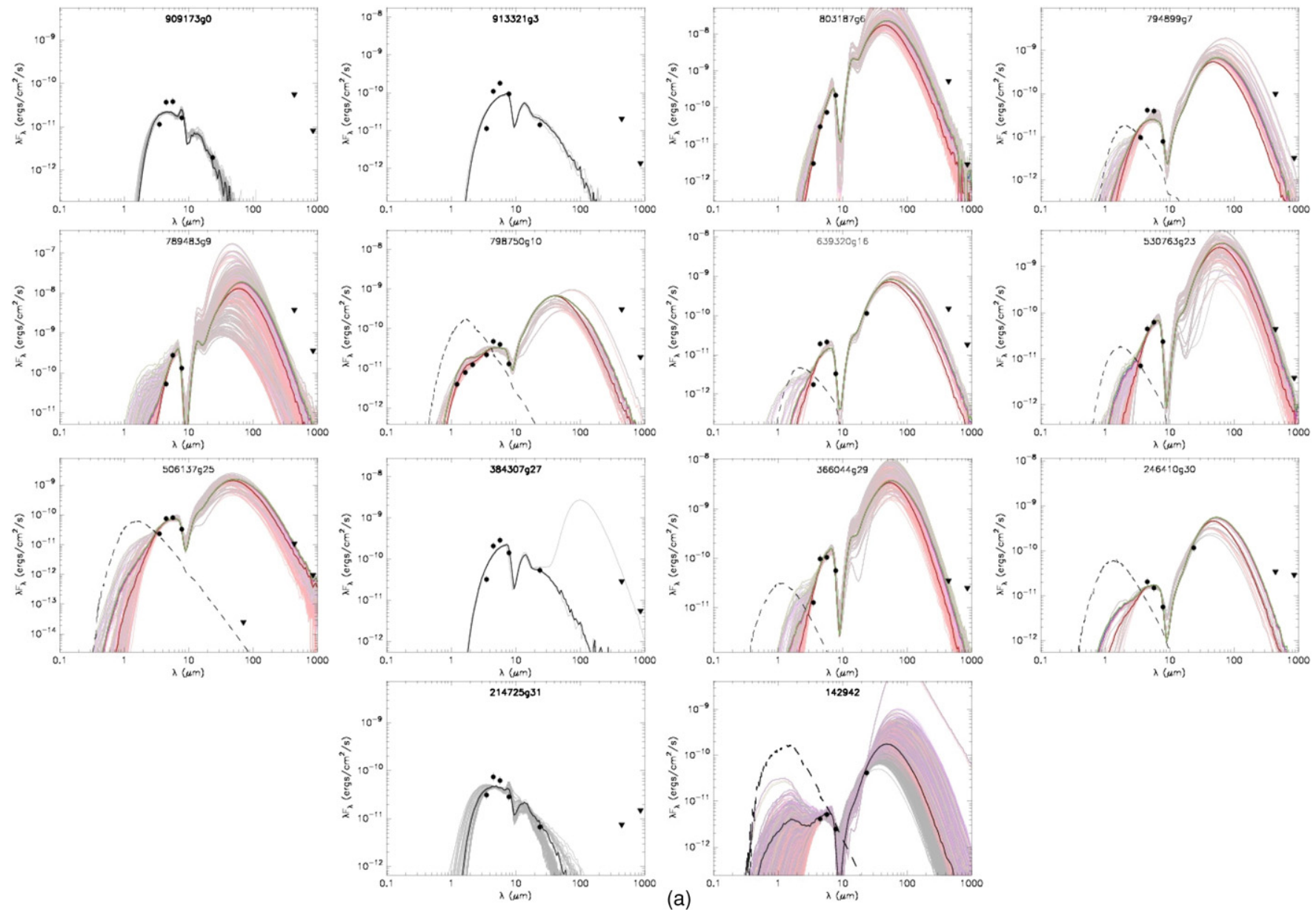

Figure 15. (a) SED fits to $4.5 \mu \mathrm{m}$ excess sources toward the Galactic center. The colored lines (reddish) correspond to fits made from different apertures. The gray lines are for IRAC sizes, and pink for $24 \mu \mathrm{m}$. (b) SED Fits to foreground $4.5 \mu \mathrm{m}$ excess sources.

(A color version of this figure is available in the online journal.)

power law of the chosen IMF. SFRs for YSOs at negative and positive longitudes are estimated to be 0.1 and $0.05 M_{\odot} \mathrm{yr}^{-1}$, respectively. The SED fitting of YSOs confirms a $10^{5} \mathrm{yr}$ duration of star formation because the number of Stage I YSO candidates dominates over Stage II and III YSOs. This implies an asymmetric SFR with respect to the Galactic center. The cause of this asymmetry could be due to a short-lived random event causing a lop-sided SFR or it might be due to a more stable structure, possibly related to the bar.

The mass of molecular gas in the central region allows us to determine the SFR per unit mass and compare it with the efficiency of star formation elsewhere in the Galaxy. The observed anticorrelation of molecular clouds and the population of YSO candidates suggest that molecular clouds have already been consumed in the formation of YSOs. If we make an assumption that a fraction of the molecular mass presently distributed in the central region is gone into formation of YSO candidates, and adopt an initial molecular gas mass of $10^{6}$ $10^{7} M_{\odot}$, then the star formation efficiency is estimated to be $\times 10^{-7}-10^{-8} \mathrm{yr}^{-1}$. The total mass of molecular gas is estimated to be $\sim 5 \times 10^{7} M_{\odot}$ (Pierce-Price et al. 2000). The estimated star formation efficiency of the Galactic disk is $5 \times 10^{-9} \mathrm{yr}^{-1}$ (Güsten \& Philipp 2004). The dominance of Stage I YSO candidates relative to Stage II suggests that a burst of star formation must have occurred $\sim 10^{5} \mathrm{yr}$ ago, consistent with our estimate above being higher than the average star formation efficiency. The global properties of interstellar and stellar components of the Galactic center region also suggest star formation occurs in bursts (Tutukov \& Kruegel 1978; Loose et al. 1982).

SFR Using Thermal Radio Measurements. If the candidate YSOs are massive and are sufficiently evolved, they are likely to produce $\mathrm{H}$ II regions that can be detected at radio wavelengths if their spectral type is earlier than B3 stars (Felli et al. 2000). To examine further the nature of infrared sources that have radio continuum counterparts, the masses inferred from radio data are compared with those inferred from SED fitting. Assuming the fluxes given in Table 1 are $\mathrm{H}$ II regions and are produced by optically thin bremsstrahlung radiation, then we can estimate the lower limit to the number of ionizing photons to sustain the ionization. As given by Condon (1992),

$$
\begin{aligned}
N_{\mathrm{Ly}}= & 6.3 \times 10^{52} \mathrm{~s}^{-1}\left(T_{e} / 10^{4}\right)^{-0.45} \\
& \times(\mathrm{v} / G H z)^{0.1}\left(L_{\text {thermal }} / 10^{27} \mathrm{erg} \mathrm{s}^{-1} \mathrm{~Hz}^{-1}\right),
\end{aligned}
$$

where $T_{e}$ is the electron temperature, $v$ is the observed frequency, and $L_{\text {thermal }}$ is the observed luminosity. The number of Lyman continuum photons per second calculated from $5 \mathrm{GHz}$ data for radio sources 5 and 6 in Table 1 are 3.6 and $3.4 \mathrm{mJy}$ corresponding to $N_{\mathrm{Ly}} \sim 1.5 \times 10^{46}$. These rates are consistent with B2 zero-age main sequence (ZAMS) stars (Vacca et al. 1996; Panagia 1973). The average masses estimated from SED fitting for sources 5 and 6 are 8.8 and $9.6 M_{\odot}$, respectively. 

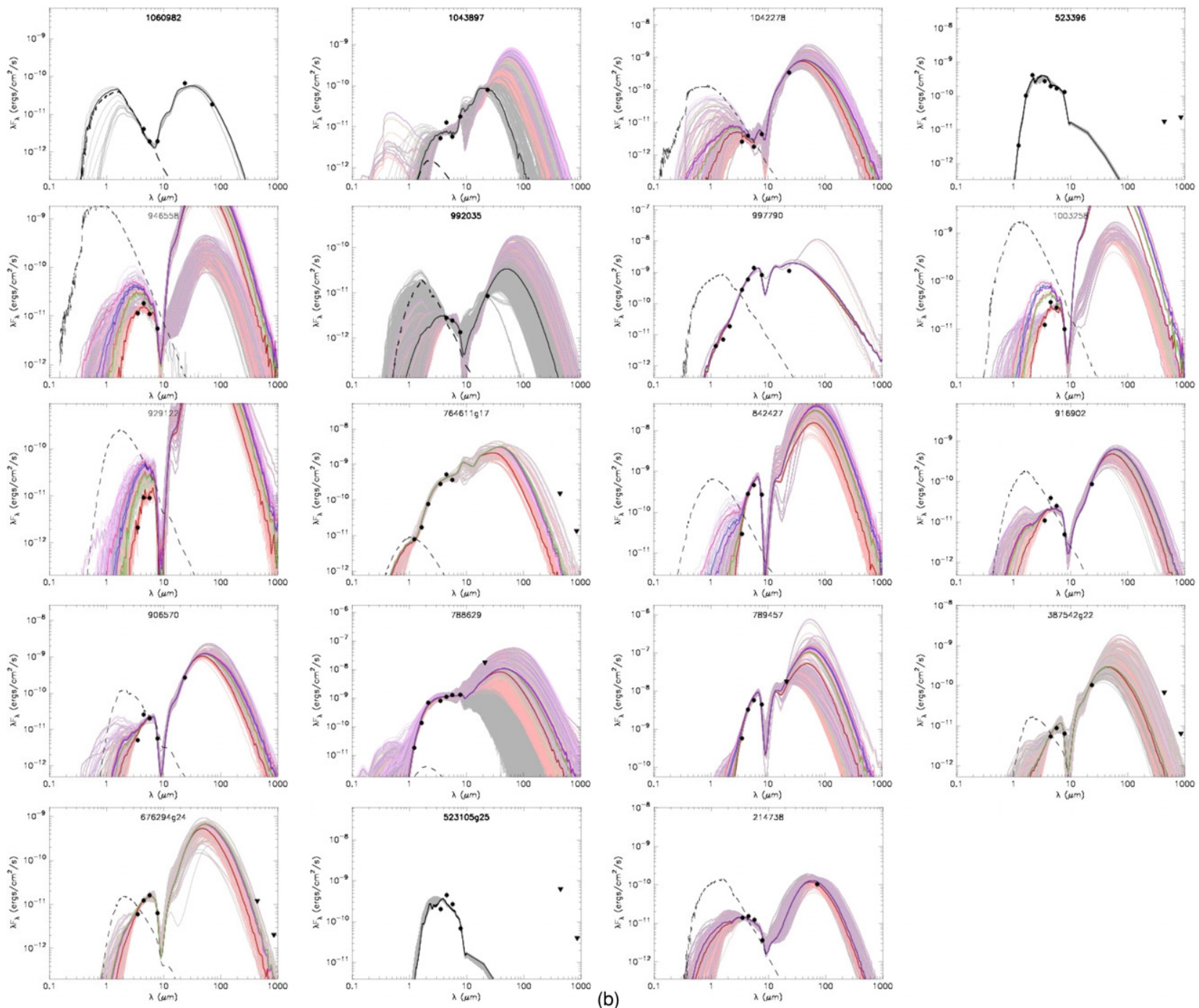

Figure 15. (Continued)

The discrepancy between the inferred mass from free-free continuum flux and from SED fitting may come from the fact that these YSOs are more evolved and SED modeling is not accounting for the Lyman continuum flux and therefore, mass determination may not be accurate. An additional uncertainty is that some of the YSO candidates may not be YSOs. These sources are likely to be found in high galactic latitudes as well as in the regions where there is no evidence for dense molecular clouds or IRDCs.

The resulting population of high-mass stars produces ionizing photons and associated free-free emission. For our adopted IMF, this population produces ionizing photons at a rate $Q \approx$ $3 \times 10^{50} \mathrm{~s}^{-1}$ and a bremsstrahlung flux of $\sim 50 \mathrm{Jy}$ at $5 \mathrm{GHz}$. Recent single-dish radio observations of this region shows a flux density of $\sim 10^{3} \mathrm{Jy}$ at $5 \mathrm{GHz}$ which includes both thermal and nonthermal emission (Law et al. 2008). The large scale $4^{\circ} \times 1^{\circ}$ radio study of this region shows that only $\sim 20 \%$ of the flux at $5 \mathrm{GHz}$ is likely to be thermal. Thus, the YSO population may contribute up to a quarter of the observed thermal emission from the Galactic center region assuming that the fraction of thermal to nonthermal emission is the same in the central $400 \times 50 \mathrm{pc}$ $\left(|l|<1.3\right.$ and $\left.|b|<10^{\prime}\right)$ as it is in the central $4^{\circ} \times 1^{\circ}$. The remaining thermal flux of $\sim 150$ Jy may be associated with highly evolved $\mathrm{H}$ II regions resulting from earlier generations of

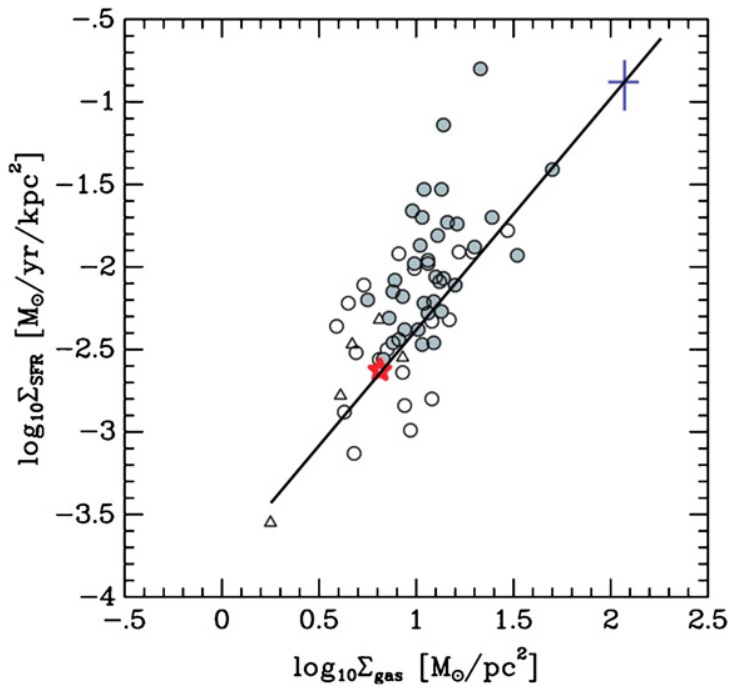

Figure 16. Correlation of the SFR with the gas surface density (Fuchs et al. 2009; Kennicutt 1998). Sa-Sb galaxies are shown as open triangles, $\mathrm{Sb}-\mathrm{Sc}$ as open circles, and Sc-Sd as filled circles. The red star is the value for the solar neighborhood and the blue cross is that for the Galactic center. The black line has a slope of 1.4, corresponding to the Schmidt-Kennicutt relationship found for external galaxies (Kennicutt 1998).

(A color version of this figure is available in the online journal.) 

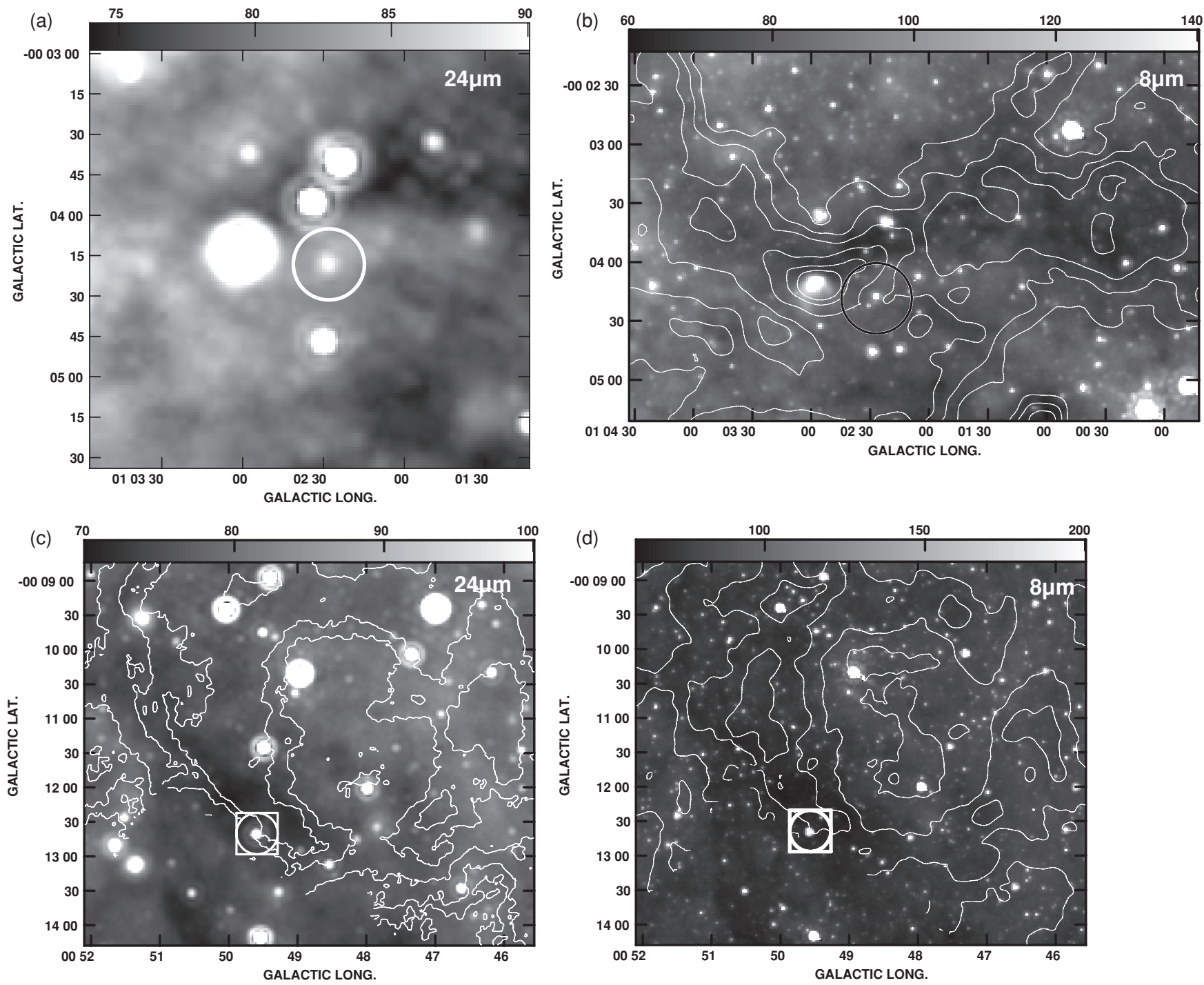

Figure 17. (a) $24 \mu \mathrm{m}$ image of the $4.5 \mu \mathrm{m}$ excess source G1.041-0.072 (g0). The circle represents the position of the $4.5 \mu \mathrm{m}$ excess source which coincides with a $24 \mu \mathrm{m}$ source. (b) Similar to (a) except that this $8 \mu \mathrm{m}$ image shows a segment of an IRDC ridge and g0. Contours of $850 \mu \mathrm{m}$ based on SCUBA observations are also superimposed on the figure. Levels are at 1.750, 2, 2.25, 2.5, 2.75, 3, and $4 \mathrm{Jy}$ beam ${ }^{-1}$. The circle marks the position of the $4.5 \mu \mathrm{m}$ excess sources whereas the square represents sources that are observed at $70 \mu \mathrm{m}$. The distribution of class I and II methanol masers are also drawn on these figures as plus $(+)$ and cross $(\times)$ signs, respectively. These symbols apply for Figures 16-35. (c) Contours of $450 \mu \mathrm{m}$ emission are superimposed on a $24 \mu \mathrm{m}$ image of g3. The location of g3 is shown by a circle whereas the square sign shows the location at which $70 \mu \mathrm{m}$ observation was made. Levels at $6,7,9,12,14$, and $20 \mathrm{Jy}$ beam ${ }^{-1}$. (d) Similar to (c) except that contours of $850 \mu \mathrm{m}$ emission are superimposed on an $8 \mu \mathrm{m}$ image of g3. Levels at 2, 2.5, 3, 3.5, 4, 4.5 Jy beam ${ }^{-1}$.

star formation activity. This point will be discussed further in Section 7.

\section{6. $4.5 \mu \mathrm{m}$ EXCESS SOURCES}

In previous sections, we have identified YSO candidates in the Galactic center region by studying the CMDs of the point sources in the surveyed region. We then refined the list of selected point sources from the CMD. The SED fitting of these selected sources used IRAC, MIPS, and 2MASS data. These YSOs are identified to be mainly of Stage I with an age of $\sim 10^{5}$ yr. We now identify YSOs that may be in a phase of star formation younger than $\sim 10^{5} \mathrm{yr}$ by examining the color of their emission in the IRAC bands. Recent studies have identified "green fuzzies" on the basis of excess emission at $4.5 \mu \mathrm{m}$ (Chambers et al. 2009; the $4.5 \mu \mathrm{m}$ sources are also known as "extended green objects (EGSs)"; Cyganowski et al. 2008). Active cores show a correlation between $24 \mu \mathrm{m}$ point sources and the "green fuzzies" whereas a quiescent core shows no IR emission. The $4.5 \mu \mathrm{m}$ excess emission is considered to be due to shock excited $\mathrm{H}_{2} \mathrm{O}-0 \mathrm{~S}(9)$ line emission (Noriega-Crespo et al. 2004 ) or the $\mathrm{CO} v=1-0$ rovibrational bandhead (Marston et al. 2004). Using this color selection criterion, a recent IRAC survey of the Galactic plane has identified a number of interacting SNRs showing excess emission at $4.5 \mu \mathrm{m}$, indicating shocked, excited $\mathrm{CO}$ and $\mathrm{H}_{2}$ molecular line emission (Reach et al. 2006). A strong correlation between shocked molecular $\mathrm{H}_{2}$ gas and methanol masers has also been noted (Lee et al. 2001), suggesting outflow is taking place at a very early stage of massive star formation, prior to the formation of a $\mathrm{H}$ II region. Also, more recently, the $4.5 \mu \mathrm{m}$ excess emission has been detected in DR21, a massive star formation site, in which the excess $4.5 \mu \mathrm{m}$ color is considered to be due to a shocked molecular outflow (Marston et al. 2004; Smith et al. 2006; Cyganowski et al. 2008) or associated with high-mass protostars within the cores of IRDCs (Rathborne et al. 2005; Beuther et al. 2005). 

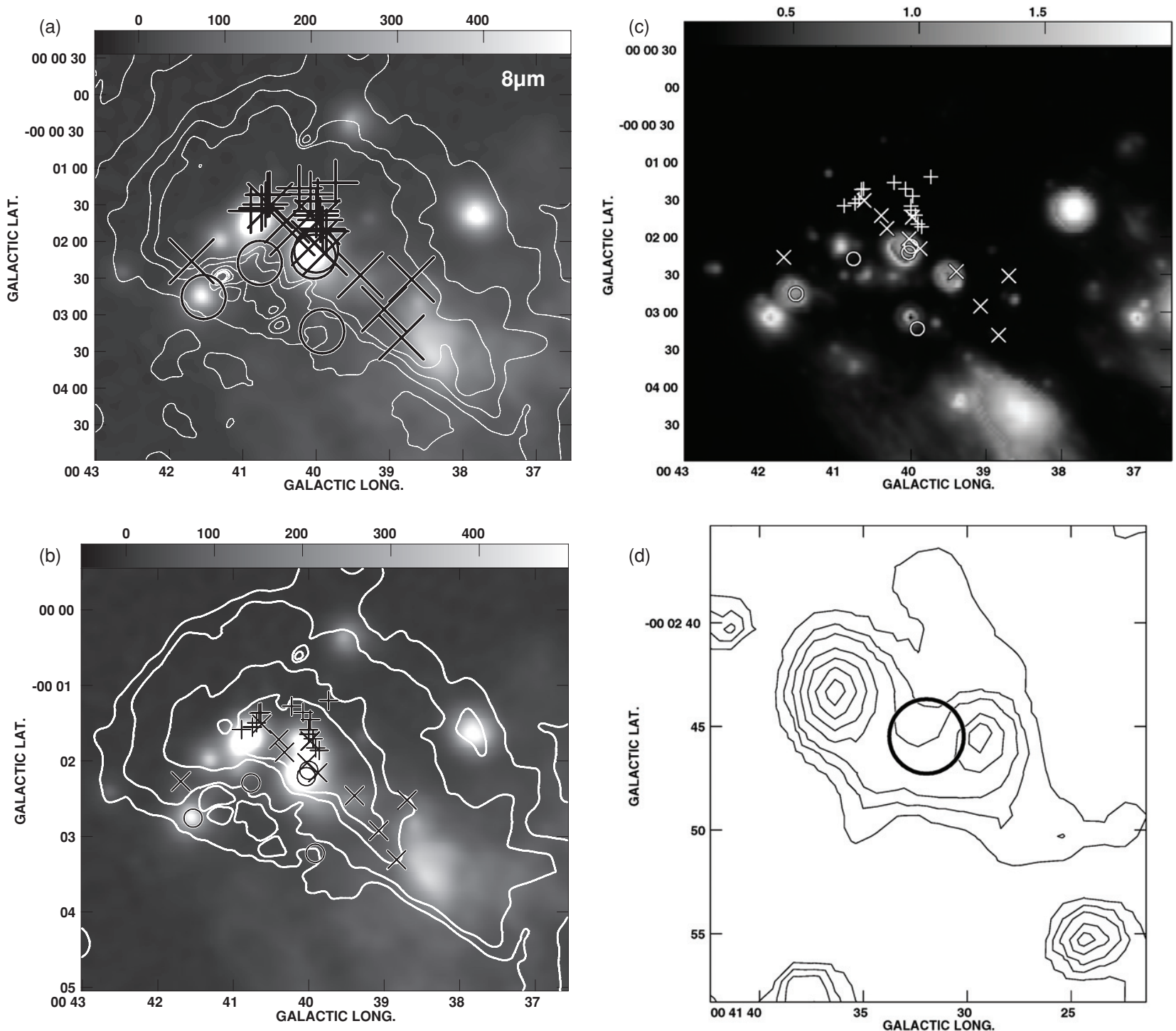

Figure 18. (a) Contours of $450 \mu \mathrm{m}$ emission superimposed on an $8 \mu \mathrm{m}$ image of Sgr B2 sources. Levels are at 35, 40, 50, 100, 200, and 400 Jy beam ${ }^{-1}$. (b) Similar to (a) except that $850 \mu \mathrm{m}$ contours are superimposed on a $20 \mathrm{~cm}$ radio continuum image. Levels are at 6, 7.5, 15, and $25 \mathrm{Jy}_{\text {beam }}{ }^{-1}$. Symbols are as defined in Figure 17 . (c) A $24 \mu \mathrm{m}$ gray-scale image of Sgr B2. (d) Contours of $4.5 \mu \mathrm{m}$ emission from the $4.5 \mu \mathrm{m}$ excess source g6. Levels are at $25,35,50,75,100,125 \mathrm{MJy} \mathrm{sr}^{-1}$. Symbols are as defined in Figure 17.

\subsection{Correlation with IRDCs}

Since there is a great deal of molecular material as traced by IRDCs in the nuclear disk, we searched for excess emission at $4.5 \mu \mathrm{m}$ ("green" in three-color images combining 3.6, 4.5, and $8 \mu \mathrm{m}$ emission) that traces molecular line emission. A quantified indication of excess $4.5 \mu \mathrm{m}$ emission is obtained by constructing the ratio $I(4.5) /\left[I(3.6)^{1.2} \times I(5.8)\right]^{0.5}$. This essentially is the ratio of the actual $4.5 \mu \mathrm{m}$ intensity to that determined by a power-law interpolation of the 3.6 and $5.8 \mu \mathrm{m}$ intensities. It was found empirically that a slight $(\sim 10 \%)$ modification to the interpolation coefficients helps emphasize sources with unusually strong $4.5 \mu \mathrm{m}$ emission. In a map of this ratio across the entire IRAC survey of the Galactic center, most point sources exhibit a uniform ratio that is not very different from the background. In regions of high extinction (e.g., IR dark clouds, and Sgr B2), this ratio rises because the reddening reduces the $3.6 \mu \mathrm{m}$ intensity. However, there were of order 100 sources, located in regions of both high and low extinction that exhibit ratios distinctly higher than those of other sources in their vicinity (within several arc minutes). These include 33 sources that were visually selected as "green" sources in three-color images. Their individual color images are shown in Figure 13; the $4.5 \mu \mathrm{m}$ excess sources show both extended and compact emission. The ratio also clearly identifies a number of interesting sources that do not stand out in the three-color images. Our search should be compared with two recent studies. Using an algorithm that finds "green fuzzies," Chambers et al. (2009) identified excess $4.5 \mu \mathrm{m}$ emission by confining to the cores of IRDCs. Another study by Cyganowski et al. (2008) visually identify "EGOs." Our technique is sensitive to both compact and extended sources with $4.5 \mu \mathrm{m}$ excess emission, unlike other searches.

The spatial distribution of the $4.5 \mu \mathrm{m}$ excess sources is marked on Figure 14(a) which displays the ratio image of the region surveyed by IRAC. There are 33 sources that are characterized to have $4.5 \mu \mathrm{m}$ excess emission, three of which (g1, g2, and g4) coincide with known planetary nebulae (PNe; Jacoby \& Van de Steene 2004). Thus, there are 30 sources that are candidate YSOs. It is quite possible that the list of $4.5 \mu \mathrm{m}$ 

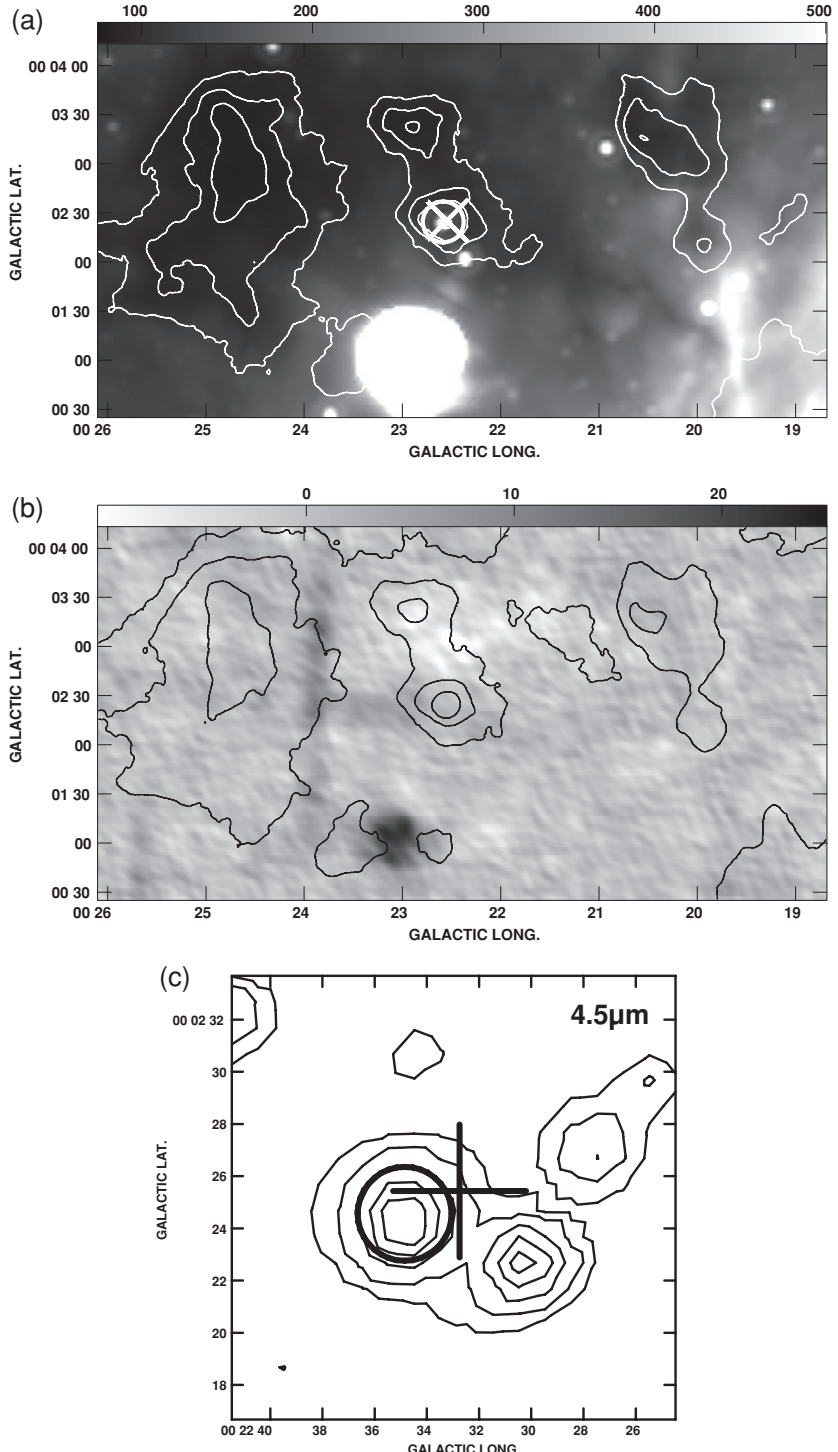

Figure 19. (a) Contours of $450 \mu \mathrm{m}$ emission around g16 are superimposed on a $24 \mu \mathrm{m}$ gray-scale image. Levels are at $3,4,5.5$, and $8 \mathrm{Jy}$ beam $^{-1}$. (b) Similar to (a) except that contours of $850 \mu \mathrm{m}$ emission are superimposed on a gray-scale $90 \mathrm{~cm}$ image. Levels are at $15,20,27.5$, and $40 \mathrm{Jy}^{\text {beam }}{ }^{-1}$. (c) A $4.5 \mu \mathrm{m}$ contours of emission from the region where g16 is identified. Levels are at 20 , 30, 50, 70, 90, and $110 \mathrm{MJy} \mathrm{sr}^{-1}$. Symbols are as defined in Figure 17.

excess sources is contaminated by additional $\mathrm{PNe}$, distributed in the crowded region of the Galactic center. Half of the $4.5 \mu \mathrm{m}$ excess sources are near Sgr B2, G0.25+0.01, M-0.02-0.07, and Sgr C (G359.5-0.0). The rest appear to be distributed away from the Galactic plane, most likely associated with local objects distributed at negative latitudes. Although there is no bias in the selection of $4.5 \mu \mathrm{m}$ excess sources toward IRDCs, Figure 14(a) shows that most of the $4.5 \mu \mathrm{m}$ excess sources are distributed in the vicinity of IRDCs. The highest density of $4.5 \mu \mathrm{m}$ excess sources is near Sgr B2.

\subsection{Correlation with Methanol Masers}

To correlate the position of highly embedded YSO candidates with early sites of massive star formation, as traced by methanol masers, Figure 14(b) presents the spatial distribution of the known methanol masers on an $8 \mu \mathrm{m}$ image. A survey of the inner $2^{\circ}$ of the Galactic center showed a total of 23 class II
Table 5

Table of Sources ${ }^{\mathrm{a}}$ with $4.5 \mu \mathrm{m}$ Excess Emission

\begin{tabular}{|c|c|c|c|c|}
\hline Source & $\begin{array}{c}\text { Galactic } \\
(l b)\end{array}$ & $\begin{array}{l}\alpha_{J 2000} \\
(\mathrm{~h} \mathrm{~m} \mathrm{~s})\end{array}$ & $\begin{array}{c}\delta_{J 2000} \\
\left({ }^{\prime \prime \prime \prime}\right)\end{array}$ & Description \\
\hline 0 & $1.041-0.072$ & 174821.37 & -280500.7 & Point \\
\hline 1 & $0.955-0.786$ & 175056.34 & -283124.4 & PN, extended? \\
\hline 2 & $0.868-0.697$ & 175023.32 & -283311.6 & PN, point \\
\hline 3 & $0.826-0.211$ & 174823.59 & -282020.7 & Point \\
\hline 4 & $0.780-0.740$ & 175021.09 & -283903.2 & PN, extended \\
\hline 5 & $0.707+0.408$ & 174542.73 & -280712.1 & Extinction of a dark cloud? \\
\hline 6 & $0.692-0.046$ & 174726.27 & -282206.7 & Extended \\
\hline 7 & $0.679-0.038$ & 174722.65 & -282231.6 & Point \\
\hline 8 & $0.667-0.037$ & 174720.60 & -282306.6 & Extended? \\
\hline 9 & $0.667-0.035$ & 174720.20 & -282305.7 & Point \\
\hline 10 & $0.665-0.054$ & 174724.28 & -282344.2 & Point \\
\hline 11 & $0.542-0.476$ & 174845.67 & -284310.9 & Point \\
\hline 12 & $0.517-0.657$ & 174924.74 & -285002.0 & Point \\
\hline 13 & $0.483-0.701$ & 174930.15 & -285306.8 & Point \\
\hline 14 & $0.477-0.727$ & 174935.52 & -285415.8 & Point \\
\hline 15 & $0.408-0.505$ & 174833.53 & -285056.1 & Extended \\
\hline 16 & $0.376+0.040$ & 174621.44 & -283537.9 & Extended \\
\hline 17 & $0.315-0.201$ & 174709.17 & -284617.8 & Point \\
\hline 18 & $0.167-0.445$ & 174745.38 & -290129.7 & Point? \\
\hline 19 & $0.091-0.663$ & 174825.71 & -291207.4 & Extended (+point) \\
\hline 20 & $0.084-0.642$ & 174819.86 & -291149.2 & Point \\
\hline 21 & $359.972-0.459$ & 174720.94 & -291154.0 & Extended (+point) \\
\hline 22 & $359.939+0.170$ & 174448.62 & -285359.1 & Extended \\
\hline 23 & $359.932-0.063$ & 174542.23 & -290138.7 & Point \\
\hline 24 & $359.907-0.303$ & 174635.04 & -29 1023.2 & Point \\
\hline 25 & $359.841-0.080$ & 174533.04 & -290649.4 & Point \\
\hline 26 & $359.614-0.245$ & 174539.29 & -292335.3 & Extended \\
\hline 27 & $359.599-0.032$ & 174447.27 & -291741.4 & Point \\
\hline 28 & $359.570+0.270$ & 174332.36 & -290940.8 & Point \\
\hline 29 & $359.437-0.102$ & 174440.46 & -292810.6 & Extended \\
\hline 30 & $359.300+0.033$ & 174348.85 & -293055.2 & Extended \\
\hline 31 & $359.199+0.041$ & 174332.35 & -293551.1 & Point \\
\hline 32 & $358.980+0.084$ & 174250.45 & -294542.2 & 2 points \\
\hline
\end{tabular}

Note.

a Visually identified from IRAC data. Many point sources may be multiple or extended sources at higher resolution.

Table 6

Table of $70 \mu \mathrm{m}$ Sources

\begin{tabular}{lcccccc}
\hline \hline Source & $l$ & $b$ & $\alpha_{J 2000}$ & $\delta_{J 2000}$ & Flux $(\mathrm{mJy})$ & $\mathrm{S} / \mathrm{N}$ \\
\hline 1 & 0.95549 & -0.78554 & 175056.34 & -283124.4 & 446 & 9.19 \\
$22^{\mathrm{a}}$ & 0.83579 & +0.18444 & 174652.86 & -280734.8 & 98080 & 43.32 \\
3 & 0.82608 & -0.21067 & 174823.59 & -282020.7 & $\ldots$ & $\ldots$ \\
5 & 0.70721 & +0.40788 & 174542.73 & -280712.1 & $\ldots$ & $\ldots$ \\
$10^{\mathrm{a}}$ & 0.49639 & +0.18764 & 174604.07 & -282452.8 & 76270 & 39.54 \\
14 & 0.47696 & -0.72730 & 174935.52 & -285415.8 & $\ldots$ & $\ldots$ \\
24 & 359.90724 & -0.30319 & 174635.05 & -291023.2 & $\ldots$ & $\ldots$ \\
28 & 359.57031 & +0.26985 & 174332.37 & -290940.8 & 2540 & 6.53 \\
31 & 359.19896 & +0.04097 & 174332.35 & -293551.2 & $\ldots$ & $\ldots$ \\
\hline
\end{tabular}

Note.

${ }^{\text {a }}$ Source No. given is for table of methanol class II maser sources.

methanol masers at $6.7 \mathrm{GHz}$ (Caswell 1996). We used limited targeted surveys of class I methanol sources. The crosses show the position of class II methanol masers (Caswell 1996). The $6.7 \mathrm{GHz}$ methanol maser observations searched the region along the plane between $|l|<0.9$ and $|b|<0.5$. Table 5 lists the position of the sources that are found within our IRAC survey. Table 6 shows the positions and fluxes of a subset of $4.5 \mu \mathrm{m}$ excess sources that were observed with MIPS at $70 \mu \mathrm{m}$. 
Table 7

Table of Methanol Class I Maser Sources

\begin{tabular}{|c|c|c|c|c|c|}
\hline Source & $\begin{array}{l}\text { Galactic } \\
\qquad(l b)\end{array}$ & $\begin{array}{l}\alpha_{J 2000} \\
\text { (h m s) }\end{array}$ & $\begin{array}{l}\delta_{J 2000} \\
\left({ }^{\circ} \prime \prime\right)\end{array}$ & $\begin{array}{c}v_{\mathrm{LSR}} \\
\left(\mathrm{km} \mathrm{s}^{-1}\right)\end{array}$ & Reference \\
\hline 0 & $359.617-0.252$ & 174541.32 & -292340.5 & +19.3 & S94,V00 \\
\hline 1 & $359.898-0.066$ & 174538.21 & -290327.7 & +21 & $\mathrm{H} 90, \mathrm{~V} 00$ \\
\hline 2 & $359.982-0.075$ & 174552.01 & -285926.7 & +44.5 & $\mathrm{H} 90, \mathrm{~V} 00$ \\
\hline 3 & $0.682-0.026$ & 174720.19 & -282203.2 & +54 & M97 \\
\hline 4 & $0.678-0.023$ & 174718.80 & -282208.3 & +68 & M97 \\
\hline 5 & $0.677-0.023$ & 174718.71 & -282209.5 & +70 & M97 \\
\hline 6 & $0.679-0.026$ & 174719.72 & -282209.5 & +66 & M97 \\
\hline 7 & $0.679-0.026$ & 174719.74 & -282209.6 & +66 & M97 \\
\hline 8 & $0.678-0.025$ & 174719.39 & -282210.7 & +62 & M97 \\
\hline 9 & $0.670-0.021$ & 174717.43 & -282227.7 & +63 & M97 \\
\hline 10 & $0.668-0.023$ & 174717.40 & -282238.0 & +75 & M97 \\
\hline 11 & $0.667-0.026$ & 174718.11 & -282249.0 & +78 & M97 \\
\hline 12 & $0.662-0.020$ & 174715.98 & -282250.5 & +74 & M97 \\
\hline 13 & $0.667-0.027$ & 174718.33 & -282250.8 & +78 & M97 \\
\hline 14 & $0.666-0.028$ & 174718.44 & -282255.7 & +73 & M97 \\
\hline 15 & $0.665-0.030$ & 174718.84 & -282301.2 & +64 & M97 \\
\hline 16 & $0.664-0.031$ & 174718.85 & -282304.8 & +67 & M97 \\
\hline 17 & $0.661-0.027$ & 174717.39 & -282305.7 & +46 & M97 \\
\hline 18 & $0.661-0.029$ & 174717.83 & -282311.1 & +57 & M97 \\
\hline 19 & $0.655-0.035$ & 174718.43 & -282341.3 & +71 & M97 \\
\hline 20 & $0.542-0.855$ & 175014.74 & -285450.6 & +16.9 & S94,V00 \\
\hline
\end{tabular}

References. H90 = Haschick et al. 1990 (44 GHz); S94 = Slysh et al. 1994 (44 GHz); M97 = Mehringer \& Menten 1997 (44 GHz, multiple sources in Sgr B2); V00 = Val'tts et al. $2000(95 \mathrm{GHz})$.

Table 8

Table of Methanol Class II Maser Sources

\begin{tabular}{|c|c|c|c|c|c|}
\hline Source & $\begin{array}{l}\text { Galactic } \\
\qquad(l b)\end{array}$ & $\begin{array}{l}\alpha_{J 2000} \\
(\mathrm{~h} \mathrm{~m} \mathrm{~s})\end{array}$ & $\begin{array}{c}\delta_{J 2000} \\
\left({ }^{\prime \prime \prime}\right)\end{array}$ & $\begin{array}{c}\mathrm{V}_{\mathrm{LSR}} \\
\left(\mathrm{km} \mathrm{s}^{-1}\right)\end{array}$ & Reference \\
\hline 0 & $359.138+0.031$ & $17: 43: 25.69$ & $-29: 39: 17.4$ & -4.0 & C96,W98 \\
\hline 1 & $359.436-0.104$ & $17: 44: 40.60$ & $-29: 28: 16.0$ & -52.0 & C96 \\
\hline 2 & $359.436-0.102$ & $17: 44: 40.21$ & $-29: 28: 12.5$ & -53.6 & C96 \\
\hline 3 & $359.615-0.243$ & $17: 45: 39.09$ & $-29: 23: 30.0$ & +22.5 & C96 \\
\hline 4 & $359.970-0.457$ & $17: 47: 20.17$ & $-29: 11: 59.4$ & +23.0 & C96 \\
\hline 5 & $0.212-0.001$ & $17: 46: 07.63$ & $-28: 45: 20.9$ & +49.2 & C96,W98 \\
\hline 6 & $0.315-0.201$ & $17: 47: 09.13$ & $-28: 46: 15.7$ & +18.0 & C96,W98 \\
\hline 7 & $0.316-0.201$ & $17: 47: 09.33$ & $-28: 46: 16.0$ & +21.0 & C96 \\
\hline 8 & $0.376+0.040$ & $17: 46: 21.31$ & $-28: 35: 39.3$ & +37.0 & C96 \\
\hline 9 & $0.393-0.034$ & $17: 46: 41.12$ & $-28: 37: 05.1$ & +28.7 & $\mathrm{C} 96$ \\
\hline 10 & $0.496+0.188$ & $17: 46: 03.96$ & $-28: 24: 52.8$ & +0.8 & C96,W98 \\
\hline 11 & $0.645-0.042$ & $17: 47: 18.67$ & $-28: 24: 24.8$ & +49.1 & C96 \\
\hline 12 & $0.647-0.055$ & $17: 47: 22.07$ & $-28: 24: 42.3$ & +51.0 & C96 \\
\hline 13 & $0.651-0.049$ & $17: 47: 21.13$ & $-28: 24: 18.1$ & +48.0 & $\mathrm{C} 96$ \\
\hline 14 & $0.657-0.041$ & $17: 47: 20.08$ & $-28: 23: 47.1$ & +52.0 & C96 \\
\hline 15 & $0.665-0.036$ & $17: 47: 20.04$ & $-28: 23: 12.8$ & +60.4 & C96,W98 \\
\hline 16 & $0.666-0.029$ & $17: 47: 18.66$ & $-28: 22: 54.5$ & +72.2 & C96 \\
\hline 17 & $0.667-0.034$ & $17: 47: 19.87$ & $-28: 23: 01.3$ & +55.2 & $\mathrm{C} 96$ \\
\hline 18 & $0.672-0.031$ & $17: 47: 20.03$ & $-28: 22: 41.7$ & +58.2 & C96 \\
\hline 19 & $0.673-0.029$ & $17: 47: 19.54$ & $-28: 22: 32.6$ & +66.0 & C96 \\
\hline 20 & $0.677-0.025$ & $17: 47: 19.29$ & $-28: 22: 14.6$ & +73.4 & C96 \\
\hline 21 & $0.695-0.038$ & $17: 47: 24.76$ & $-28: 21: 43.2$ & +68.5 & C96 \\
\hline 22 & $0.836+0.184$ & $17: 46: 52.86$ & $-28: 07: 34.8$ & +3.5 & C96,W98 \\
\hline
\end{tabular}

References. C96 = Caswell 1996; W98 = Walsh et al. 1998.

It is now well established that methanol masers are signposts of ongoing massive star formation throughout the Galaxy. Class II methanol masers are recognized to be radiatively pumped, unlike class I methanol masers, known to be collisionally pumped (Menten 1991). Tables 7 and 8 show the positions of classes I and II methanol masers and their corresponding velocities, respectively. The correlation of methanol masers with the $4.5 \mu \mathrm{m}$ excess sources in the surveyed region was first reported by Yusef-Zadeh et al. (2007a). The number of $4.5 \mu \mathrm{m}$ excess sources distributed in the Galactic center region is 14 but we detected 6 methanol maser counterparts. Given the limited sensitivity of $1 \mathrm{Jy}$ in the maser survey by Caswell (1996), it is possible that many of the $4.5 \mu \mathrm{m}$ excess sources have weak maser counterparts or that they signify different evolutionary phases of massive protostars when compared with the onset of methanol maser emission. A more detailed study of the correlation of $6.7 \mathrm{GHz}$ methanol masers and EGOs with IRDCs in the GLIMPSE data was recently reported by Cyganowski et al. (2008).

\subsection{The SEDs of $4.5 \mu \mathrm{m}$ Excess Sources}

Using 2MASS, IRAC, and MIPS point-source catalogs as well as 850 and $450 \mu \mathrm{m}$ data (Pierce-Price et al. 2000), we constructed the SEDs of individual $4.5 \mu \mathrm{m}$ excess sources to be used in model fitting. Due to the lower resolution of the submillimeter data and the excess flux of molecular emission in the $4.5 \mu \mathrm{m}$ IRAC band, we show upper limits to the peak flux densities at 4.5, 450, and $850 \mu \mathrm{m}$ (Pierce-Price et al. 2000; Ramirez et al. 2008). The distances to many of the $4.5 \mu \mathrm{m}$ excess sources are unknown due to the difficulty in estimating the kinematic distances of sources in the direction toward the Galactic center. We assumed that the sources that are distributed at high galactic latitudes are most likely foreground sources whereas the range of distances for low latitude sources galactic latitudes is restricted to $|b|<10^{\prime}$ and are near the Galactic center. The model fitting for foreground sources was restricted to distances ranging between 2 and $6 \mathrm{kpc}$ whereas the range of distance for low galactic latitudes is between 7.5 and $8.5 \mathrm{kpc}$. The derived masses and luminosities of the $4.5 \mu \mathrm{m}$ excess sources based on the well-fitted SED models are shown in Tables 9 and 10 for Galactic center and foreground sources, respectively. It should be pointed out that if the fitter finds a low- $A_{v}$ model to fit a source, that does not mean that the source is not at the Galactic center. The fitter will find models that can fit a range of $A_{v}$ s even if the source is at the Galactic center by restricting this variable to reasonable values. This restriction allows the underlying YSO models to more appropriate models for fitting that source. We note that for the very young sources that we believe are associated with $4.5 \mu \mathrm{m}$ excesses, the YSO mass estimate is the current mass.

Assuming that the sources that are not SED fitted are YSOs, there are currently no available models in the grid to fit these sources. Also, the parameter "nfits" in Table 9 depends on how many data points are available. The fewer the data points, the more models can fit the data. If there are too many fits to the data points, it suggests the source is poorly constrained (which will show up in the standard deviations). On the other hand, if there are too few fits, it suggests the models are wrong (e.g., single source YSO trying to fit a cluster) or the data are bad. We note a strong correlation between the number of data points and $\chi^{2}$, as well as an inverse correlation between the number of data points and the number of "acceptable" models. And as the number of acceptable models increases, the (relative) uncertainties also increase, especially for $\left\langle L_{\star}\right\rangle$ and $\left\langle\dot{M}_{\text {env }}\right\rangle$. Nevertheless, it is clear that the number of fits and standard deviations allow us to identify well constrained sources, unlike the CMD technique. We identify ten $4.5 \mu \mathrm{m}$ sources in Stage I and four in Stage II and III evolutionary phases. The fraction of Stage I sources is $\sim 70 \%$ which is slightly lower than that found in the G359.43+0.02 cluster $(78 \%)$ and higher than the fraction of $60 \%$ found in the 

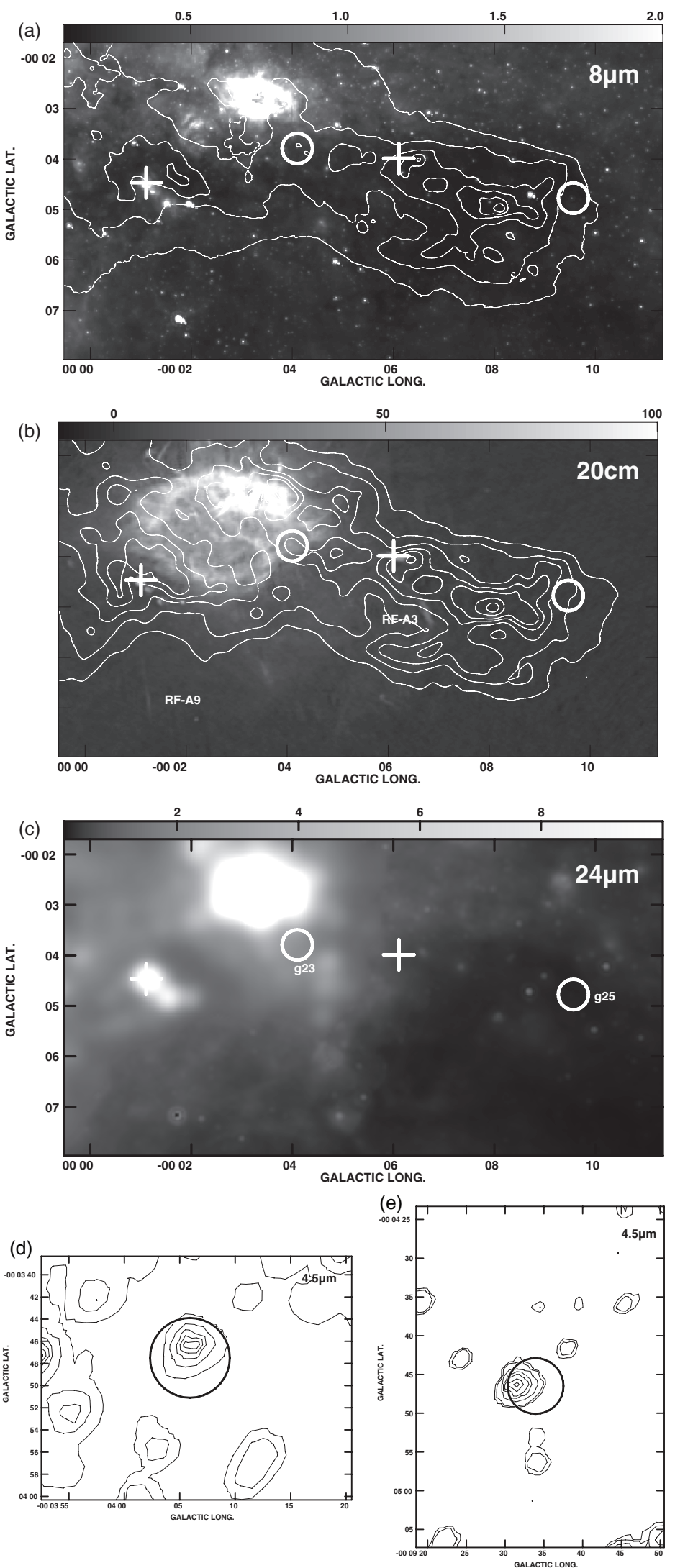

Figure 20. (a) Contours of $450 \mu \mathrm{m}$ emission from the 50 and $20 \mathrm{~km} \mathrm{~s}^{-1}$ molecular clouds are superimposed on an $8 \mu \mathrm{m}$ image of this region. The circles show the positions of $4.5 \mu \mathrm{m}$ excess sources g23 and g25, projected toward the $50 \mathrm{~km} \mathrm{~s}^{-1}$ and $20 \mathrm{~km} \mathrm{~s}^{-1}$ molecular clouds, respectively. The plus signs shown the position of class I methanol sources in this region. (b) Similar to (a) except that contours of $850 \mu \mathrm{m}$ emission are superimposed on a radio continuum image at $20 \mathrm{~cm}$. Levels are at $4,5,6,7,8,10,12$, and $14 \mathrm{Jy} \mathrm{beam}^{-1}$ (c) A $24 \mu \mathrm{m}$ image of the Sgr A region based mainly on the saturated MIPS data that are replaced by MSX data. The IRDCs associated with the 50 and $20 \mathrm{~km} \mathrm{~s}^{-1}$ are best represented in this figure. Symbols are as defined in Figure 17. (d) Contours of $4.5 \mu \mathrm{m}$ emission from g23 at levels of $50,100,200,300,400,500,600$, and $700 \mathrm{MJy} \mathrm{sr}^{-1}$. (e) Contours of $4.5 \mu \mathrm{m}$ emission from g25 at levels of 40, 50, 100, 200, 300, 500, 700 MJy sr ${ }^{-1}$. Symbols are as defined in Figure 17. 

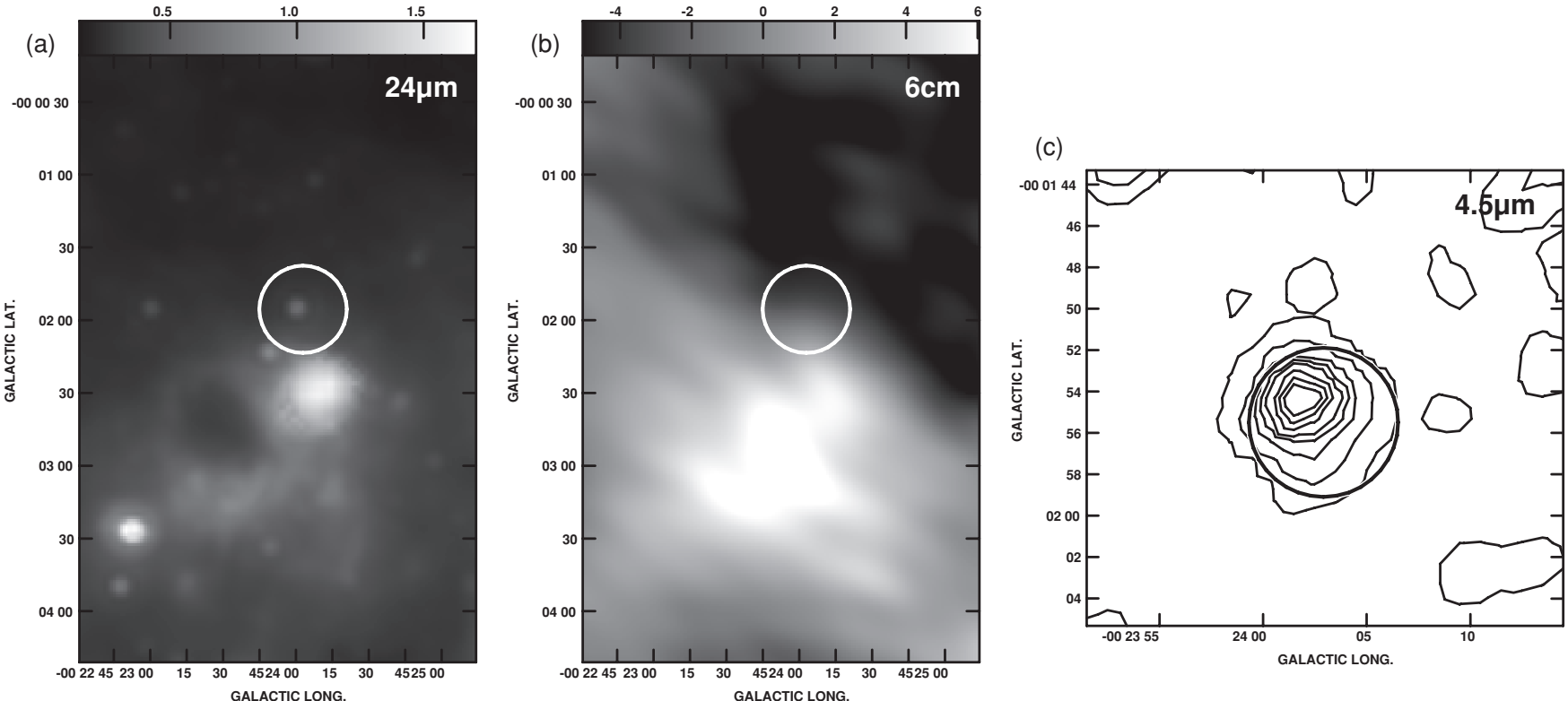

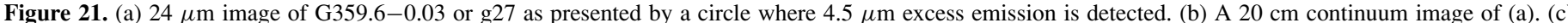

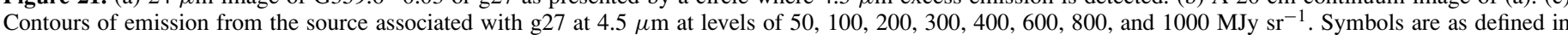
Figure 17.
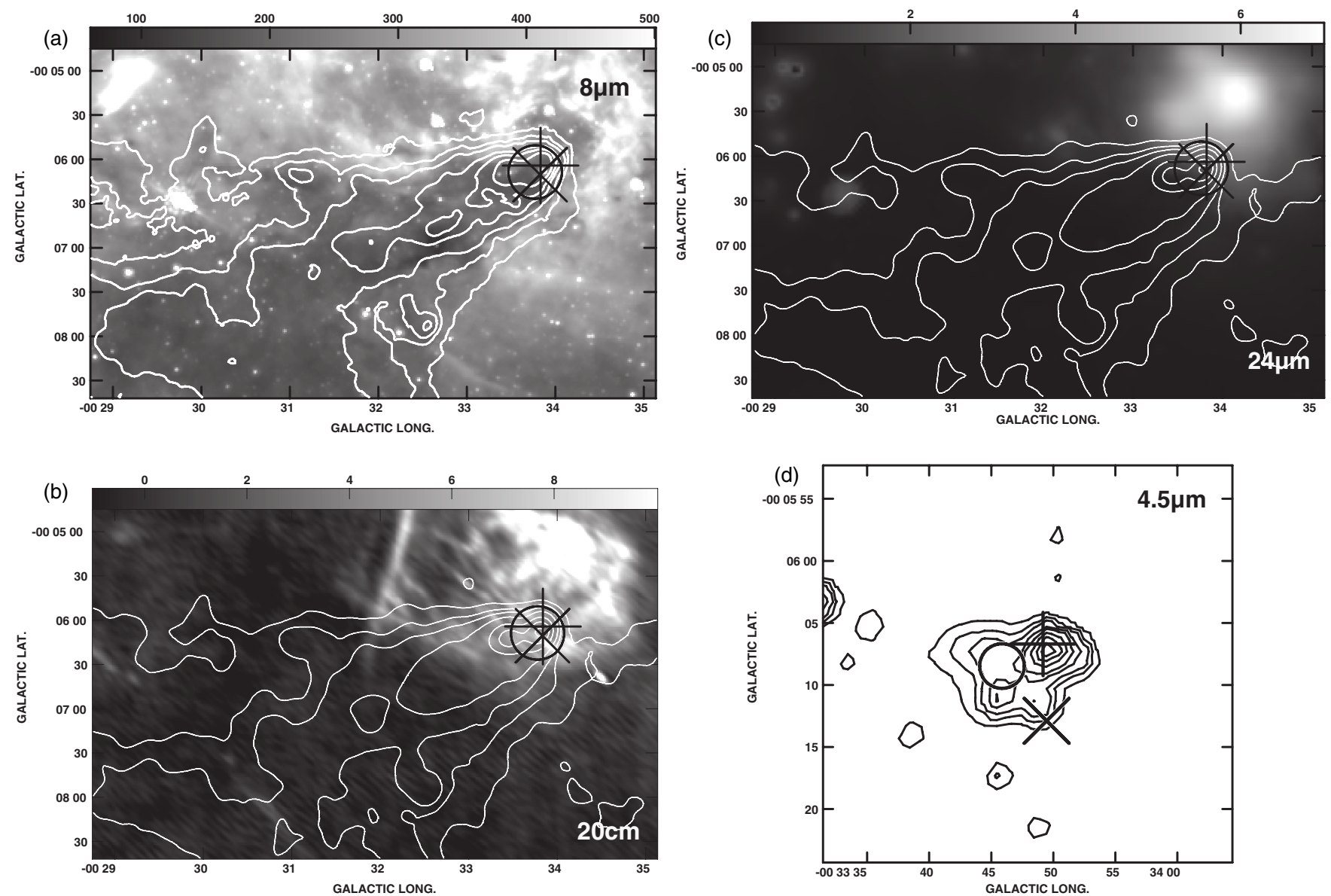

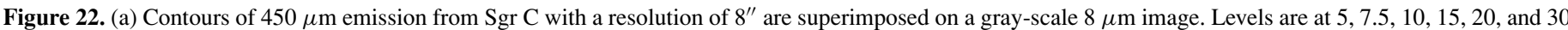

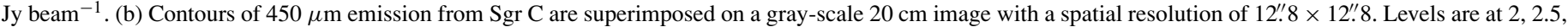

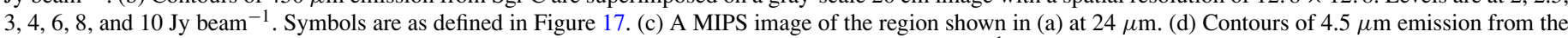
$4.5 \mu \mathrm{m}$ excess source g29, as identified by a circle. Levels are at 50, 75, 100, 150, 200, and $300 \mathrm{MJy} \mathrm{sr}^{-1}$. Symbols are as defined in Figure 17 .

region between $|b|=10^{\prime}$ and $|l|<1.3$. This is consistent with the idea that the $4.5 \mu$ m excess sources are in their early phase of evolution. The tables presented here give sufficient information for use of the fits in future studies. 

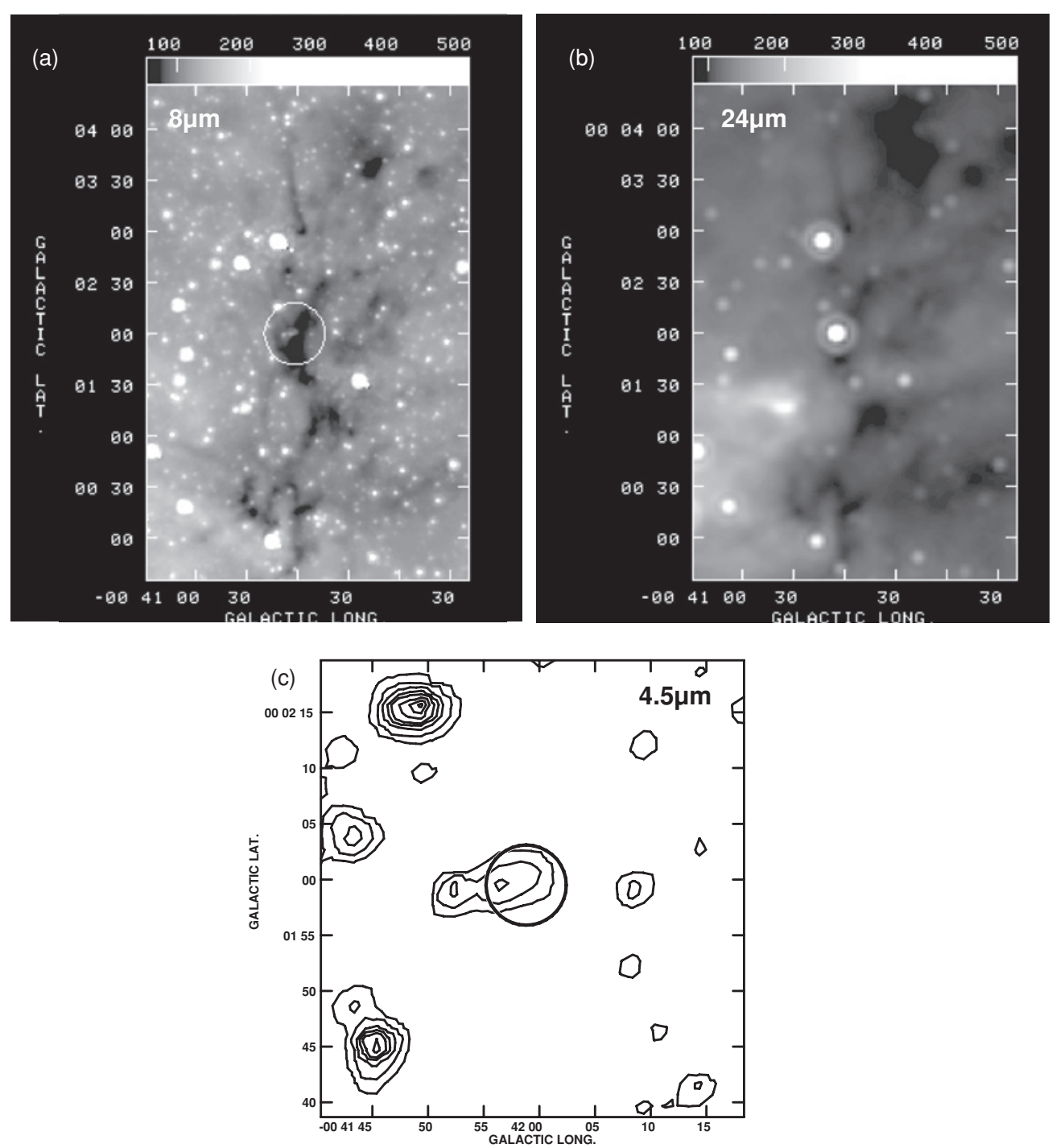

Figure 23. (a) Gray-scale $8 \mu \mathrm{m}$ image of the $4.5 \mu \mathrm{m}$ excess source g30. The position of the $4.5 \mu \mathrm{m}$ excess source is shown by a circle. (b) Similar to (a) except at $24 \mu \mathrm{m}$. (c) Contours of $4.5 \mu \mathrm{m}$ emission from G359.30+0.033 at levels of 25, 50, 100, 150, 200, 300, 400, and $500 \mathrm{MJy} \mathrm{sr}^{-1}$. Symbols are as defined in Figure 17.

The visual extinction levels derived from fitting the SEDs of the sources which are most likely located near the Galactic center range between 5 and 47 mag. Figure 15(a) shows the fitted SEDs of selected sources with high extinction. The number of good fits, as seen in Figure 15 and Table 9, depends both on the quality of the data, and the number of models that happen to have an SED that looks like the one fitted. We note from Figure 15 that $\mathrm{g} 0, \mathrm{~g} 5, \mathrm{~g} 27, \mathrm{~g} 31$ are not well fit by the models as they seem to have narrow peaks at about 4-5 $\mu \mathrm{m}$, dropping down on either side. Thus, unlike the rest, these sources are unlikely to be accreting YSOs. The correlation of $4.5 \mu \mathrm{m}$ sources and IRDCs is strong and is consistent with recent analysis by Chambers et al. (2009); we found 11 of the $4.5 \mu \mathrm{m}$ sources are correlated with IRDCs, half of them are not extended and 6 of the 14 sources have methanol maser counterparts. The correlation of the majority of $4.5 \mu \mathrm{m}$ excess sources with IRDCs, which are known to lie near the Galactic center, supports our initial choice for placing the low latitude $4.5 \mu \mathrm{m}$ excess sources at the distance of the Galactic center.

Appendix A.1 describes each of the Galactic center and foreground $4.5 \mu \mathrm{m}$ excess sources.

\section{DISCUSSION}

\subsection{Star Formation History of the Nuclear Disk}

To better understand the nature of star formation history in the complex region of the nuclear disk, we have used several different measurements. These measurements include $4.5 \mu \mathrm{m}$ excess sources, Stage I YSOs, thermal H II regions and diffuse nonthermal radio flux, each of which is described below.

We adopt, as in Section 5.3, a standard broken power-law form of the IMF (Whitney et al. 2008; Kroupa 2001) to estimate on-going SFR based on SED fitted $4.5 \mu \mathrm{m}$ excess sources. We believe these excess sources trace the earliest phase of star formation. Given a large uncertainty in the completeness of $4.5 \mu \mathrm{m}$ sources, the integrated mass of Stage I sources using Kroupa IMF, as before, is estimated to be $\sim 522 M_{\odot}$. Assuming that typical age of $4.5 \mu \mathrm{m}$ excess sources is $\sim 5 \times 10^{4}-10^{5} \mathrm{yr}$, we estimate SFR 0.017-0.009 $M_{\odot} \mathrm{yr}^{-1}$, respectively. Given the vast amount of dense molecular gas corresponding to $\sim(5) \times 10^{7} M_{\odot}$ residing in the Galactic center region, the efficiency of star formation is estimated to be $\sim(1-3.4) \times 10^{-10} \mathrm{yr}^{-1}$. 

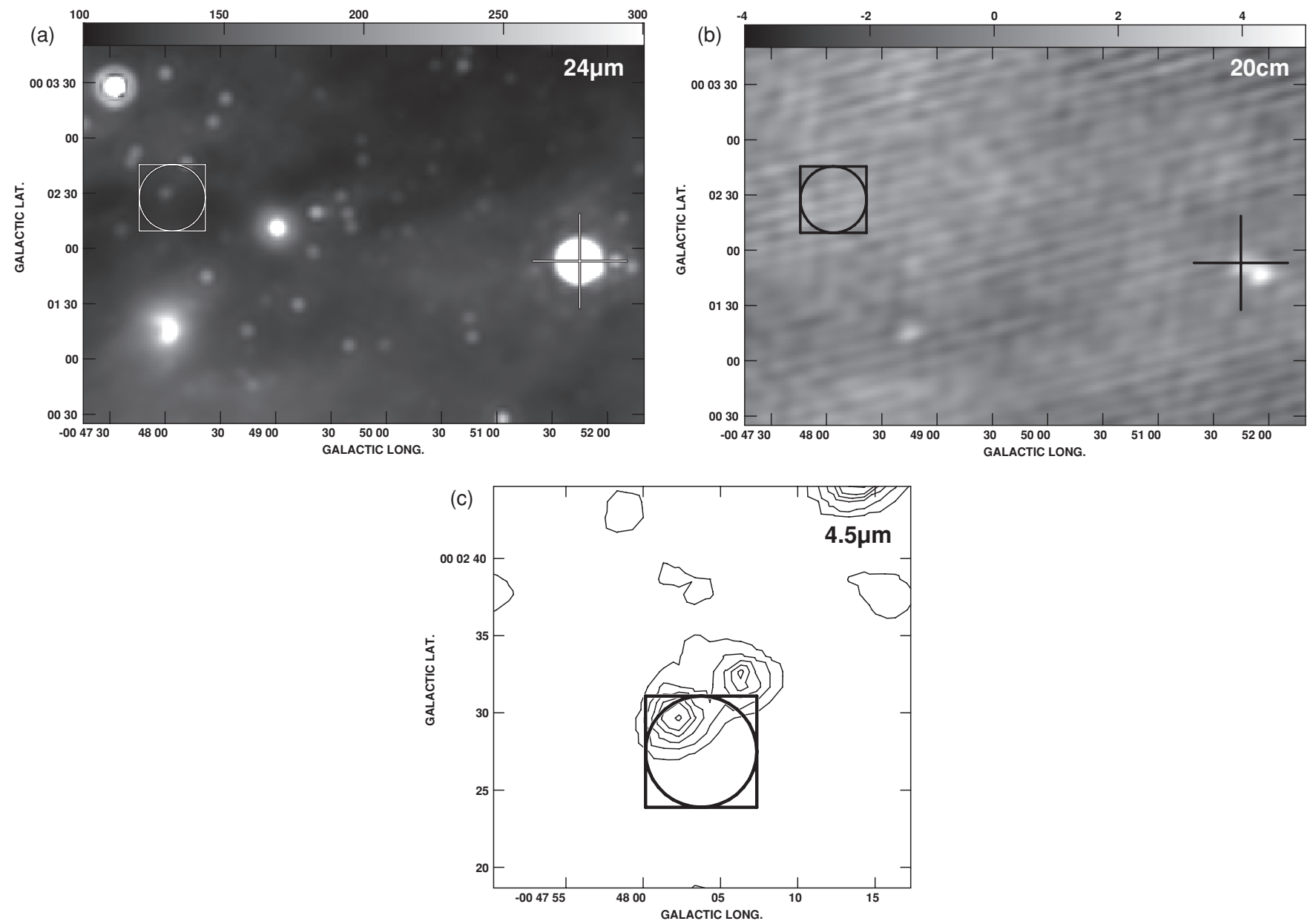

Figure 24. (a) Gray-scale $24 \mu \mathrm{m}$ image of the $4.5 \mu \mathrm{m}$ excess source g31. (b) Similar to (a) except that the image is shown at $20 \mathrm{~cm}$. (c) Contours of $4.5 \mu \mathrm{m}$ emission from G359.199+0.041 at levels of 50, 100, 200, 300, 400, 600, 800, and $1000 \mathrm{MJy} \mathrm{sr}^{-1}$. Symbols are as defined in Figure 17.

A more robust approach to estimate SFR, as discussed earlier in Section 5.3.2, is to use the evidence for a population of Stage I YSO candidates in the nuclear disk and make an estimate of the SFR $\sim 0.14 M_{\odot} \mathrm{yr}^{-1}$ in the last $10^{5} \mathrm{yr}$. The SFR during this period appears to be an order of magnitude higher that estimated from $4.5 \mu \mathrm{m}$ excess sources. These excess sources are in an earlier phase of their evolution and are younger than $10^{5} \mathrm{yr}$.

To estimate the SFR estimated from YSOs older than $10^{5} \mathrm{yr}$, we used $24 \mu \mathrm{m}$ flux density. This flux is representative of the ionizing flux and hence of ages of a few million years. We find a total $24 \mu \mathrm{m}$ flux density of $1.17 \times 10^{5} \mathrm{Jy}$ within the central region, $|l|<1.3,|b|<10^{\prime}$ (i.e., a radius of $182 \mathrm{pc}$ ). The $K$ band extinction over this region appears to average slightly more than 2 mag (Dutra et al. 2003) and the observed ratio of $A_{24} /$ $A_{K} \sim 0.5$ (Chapman et al. 2009). We therefore corrected the observed flux density for $1.1 \mathrm{mag}$ of extinction. For a distance of $8.5 \mathrm{kpc}$, the resulting $24 \mu \mathrm{m}$ luminosity is $\sim 9 \times 10^{7} L_{\odot}$. In the formulation of Rieke et al. (2009), this luminosity implies a SFR of $0.07 M_{\odot} \mathrm{yr}^{-1}$, assuming a Kroupa IMF. This estimate implicitly assumes that the SED fitted YSOs did not contribute significantly to the total measured flux at $24 \mu \mathrm{m}$.

Lastly, nonthermal radio emission can be used as another proxy to determine SFR. This SFR probably applies to conditions at some tens of millions of years ago (Helou \& Bicay 1993). The flux density of nonthermal emission is estimated to be $800 \mathrm{Jy}$ at $6 \mathrm{~cm}$. If we extrapolate this flux to $21 \mathrm{~cm}$ flux assuming a power-law spectral index of 0.8 , we derive a lumi- nosity at this wavelength of $61.2 L_{\odot}$. This then yields a SFR of $0.007 M_{\odot} \mathrm{yr}^{-1}$ (Rieke et al. 2009).

Taken together the above estimates provide a reasonable picture of large-scale star formation activity in the nuclear disk. We suggest that the region within $\sim 400 \mathrm{pc}$ (diameter) of the Galactic center region was in a very quiescent state $\sim 10^{7} \mathrm{yr}$ ago, and that the SFR has been rising slowly, peaking to a value of $0.14 M_{\odot} \mathrm{yr}^{-1}$ around $10^{5} \mathrm{yr}$ ago and then perhaps falling since then to the current value of $\sim 0.01 M_{\odot} \mathrm{yr}^{-1}$.

\subsection{The Schmidt-Kennicutt Law in the Galactic Center}

A motivation for this work has been to test the influence of the extreme ISM conditions in the central $400 \mathrm{pc}$ (diameter) of the Milky Way on the process of star formation. We have found that the behavior of various SFR indicators is consistent with expectations from the global properties of other galaxies. The value of $\mathrm{q}_{24}=\log \left(\mathrm{f}_{21 \mathrm{~cm}} / \mathrm{f}_{24 \mu \mathrm{m}}\right) \sim 2.1$, where $f_{21 \mathrm{~cm}}$ and $f_{24 \mu \mathrm{m}}$ represent flux densities at $21 \mathrm{~cm}$ and $24 \mu \mathrm{m}$, respectively, is at the extreme high end of the range for external galaxies and is larger than is typically observed in the centers of nearby galaxies (Murphy et al. 2006) but can be explained in terms of a relatively low rate of star formation in the past. The properties of the individual forming massive stars also are consistent with those of similar objects elsewhere in the Galaxy.

A less ambiguous test for changes in star-forming properties is based on the Schmidt-Kennicutt Law. This law is an empirical power-law relation between the surface densities of the 

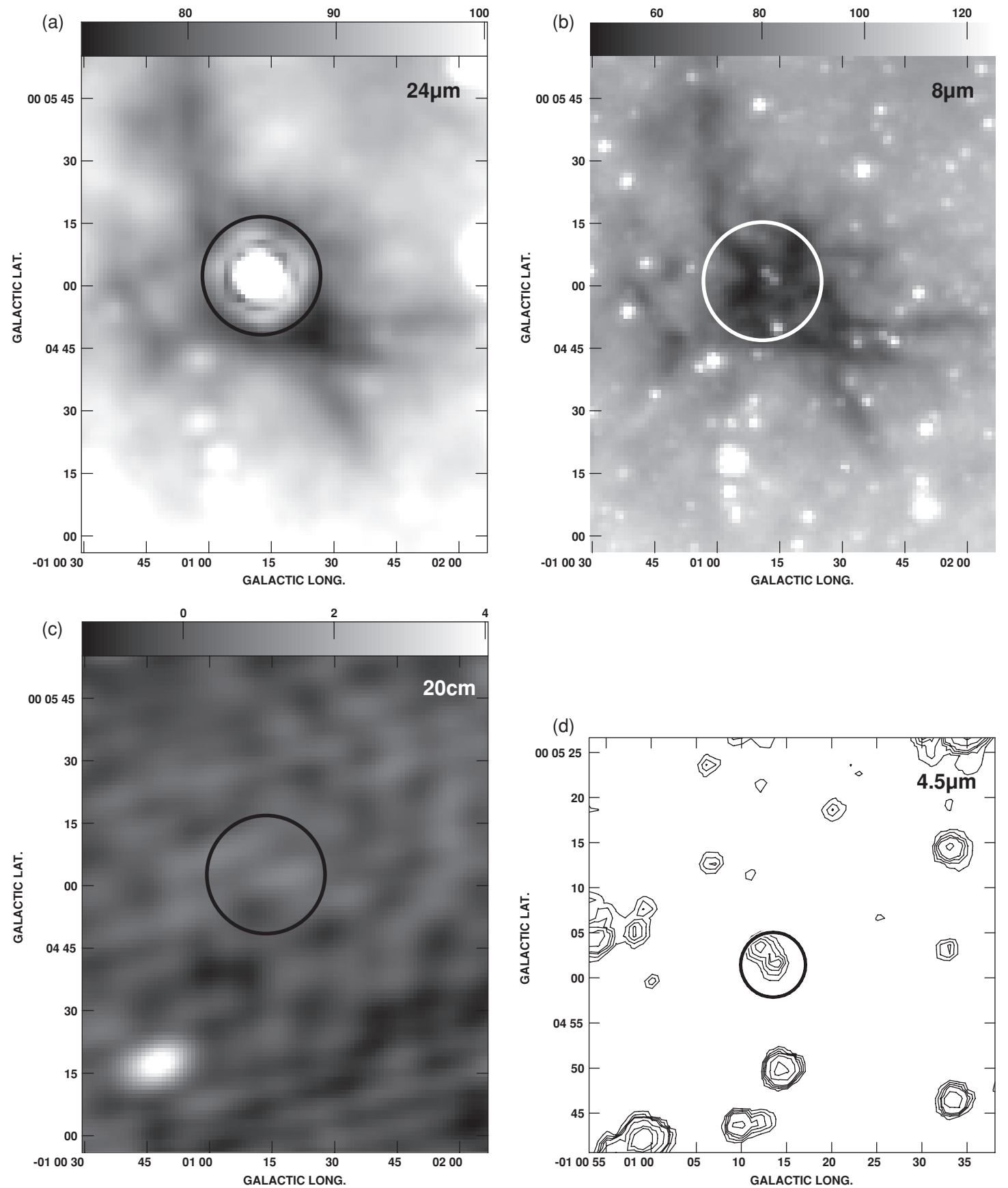

Figure 25. (a) Gray-scale $24 \mu \mathrm{m}$ image of the $4.5 \mu \mathrm{m}$ excess source $\mathrm{g} 32$ from $73 \mathrm{MJy} \mathrm{sr}^{-1}$. (b) Similar to (a) except at $8 \mu \mathrm{m}$ from 47 to $125 \mathrm{MJy}$ sr ${ }^{-1}$. (c) Similar to (a) except at $20 \mathrm{~cm}$ from range $=-1.3$ to $4 \mathrm{mJy}_{\text {beam }^{-1}}$. (d) Contours of $4.5 \mu \mathrm{m}$ emission from G359.30+0.033 at levels of 15, 20, 25, 30, 50, 100, 150, and 300 MJy sr ${ }^{-1}$. The position of the $4.5 \mu \mathrm{m}$ excess source is shown by a circle. Symbols are as defined in Figure 17.

interstellar gas and of star formation (see, e.g., Kennicutt 1998). If this law holds between the disk and center of the Milky Way, it would be strong evidence that the high interstellar gas temperatures in the latter region do not fundamentally alter the manner in which stars form there. Fuchs et al. (2009) have determined the relevant surface densities for the Milky Way disk near the sun and show that this region does indeed behave as expected according to the Schmidt-Kennicutt Law. We now estimate the same parameters for the central $0.8 \mathrm{kpc}$ of the Galaxy. We take the mass in gas from Pierce-Price et al. (2000) to be $(5.3 \pm 1.0) \times 10^{7} M_{\odot}$. Our estimates of the SFR have a range: $0.007 M_{\odot} \mathrm{yr}^{-1}$ from the nonthermal radio, $0.03 M_{\odot} \mathrm{yr}^{-1}$ from the youngest forming massive stars; $0.062 M_{\odot} \mathrm{yr}^{-1}$ from the
$24 \mu$ m luminosity; and a peak of $0.14 M_{\odot} \mathrm{yr}^{-1}$ from the $\sim 10^{5} \mathrm{yr}$ old YSO candidates. A long-term time-averaged value can also be estimated. The enclosed mass in this region is $1.5 \times 10^{9} M_{\odot}$ (Haller et al. 1996). Therefore, a strict upper limit on the average SFR over the past $10^{10} \mathrm{yr}$ is $0.15 M_{\odot} \mathrm{yr}^{-1}$; a more plausible average would be $25 \%-50 \%$ of this value. This average is in good agreement with our measurements of the recent SFR and suggests that the variations represent fluctuations around this average. We take the typical recent SFR to lie between 0.04 and $0.08 M_{\odot} \mathrm{yr}^{-1}$. In Figure 16, we plot the Galactic center values on Figure 4 of Fuchs et al. (2009). In this figure, different types of $\mathrm{Sa}, \mathrm{Sb}$, and $\mathrm{Sc}$ galaxies are shown as open triangles, open circles, and filled circles. The red star is the value for the solar 

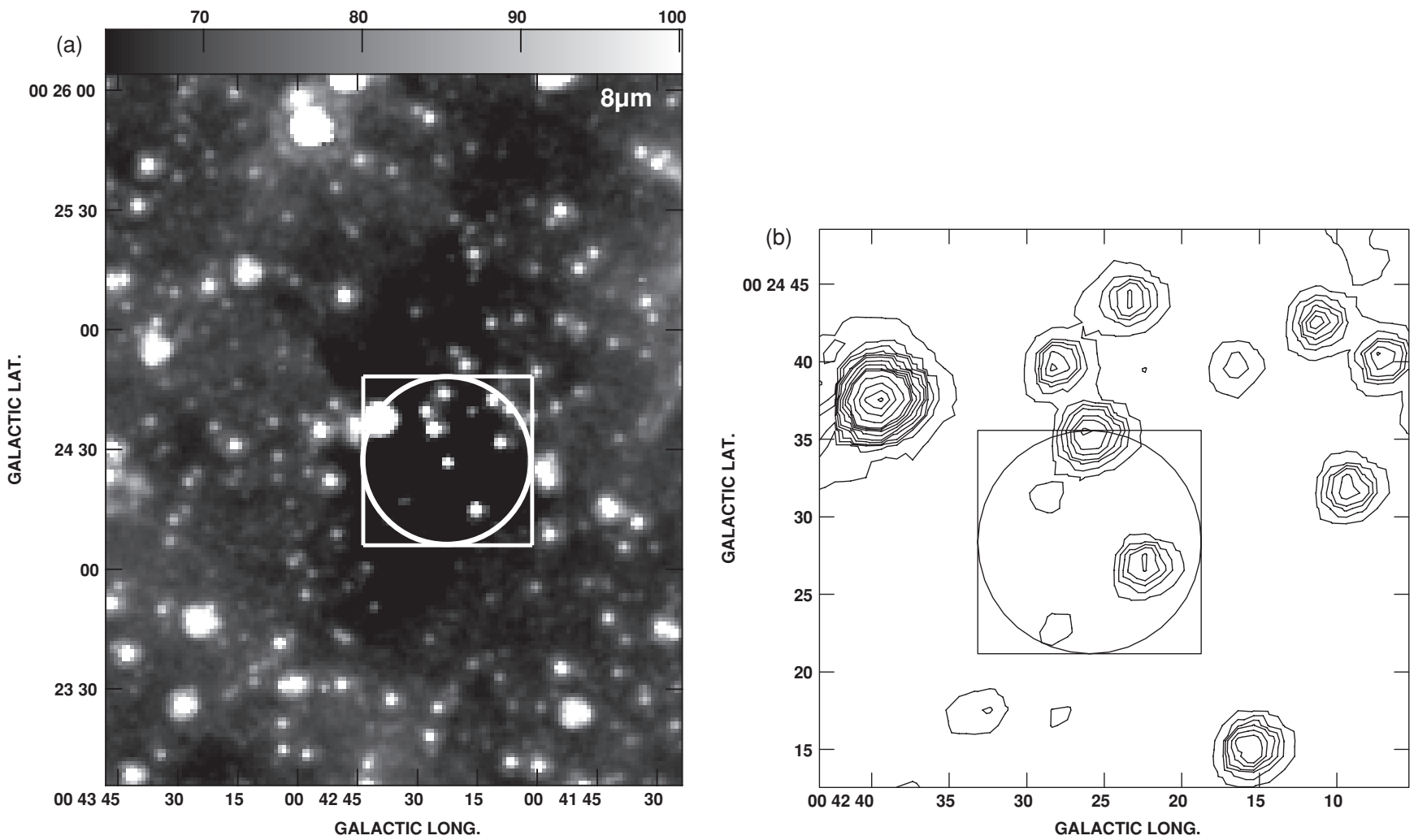

Figure 26. (a) $8 \mu \mathrm{m}$ image from 64 to $100 \mathrm{MJy} \mathrm{s}^{-1}$ of the $4.5 \mu \mathrm{m}$ excess source g5, G0.708+0.408, projected in the middle of an IR dark cloud. (b) Contours of $4.5 \mu \mathrm{m}$ emission from the $4.5 \mu \mathrm{m}$ excess source $\mathrm{g} 5$ that shows excess emission at this wavelength. Levels are at 25, 50, 75, 100, 150, 200, 300, 400, 500, 600, 1000, 2000, $2500 \mathrm{MJy} \mathrm{sr}^{-1}$. Symbols are as defined in Figure 17.

Table 9

The Parameters of SED Fits to $4.5 \mu \mathrm{m}$ Excess Sources Near the Galactic Center

\begin{tabular}{|c|c|c|c|c|c|c|c|c|c|c|}
\hline Green No. & SST GC No. & $l$ & $b$ & $\chi_{\min }^{2}$ & nfits & $\left\langle A_{v}\right\rangle$ & $\left\langle M_{\odot}\right\rangle$ & $\left\langle L_{\odot}\right\rangle$ & $\langle\dot{M}\rangle$ & $\langle$ Stage $\rangle$ \\
\hline g0 & 909173 & 1.0407 & -0.0714 & 4.36 & 31 & 42.4 & $16.1 \pm 3.5$ & $2.8 \pm 1.4 \mathrm{E}+04$ & $0.0 \pm 0.0 \mathrm{E}+00$ & III \\
\hline g3 & 913321 & 0.8262 & -0.2108 & 11.45 & 4 & 43.6 & $10.6 \pm 0.0$ & $7.6 \pm 0.0 \mathrm{E}+03$ & $0.0 \pm 0.0 \mathrm{E}+00$ & II \\
\hline g6 & 803187 & 0.6934 & -0.0451 & 1.71 & 46 & 46.7 & $29.9 \pm 8.2$ & $1.4 \pm 1.0 \mathrm{E}+05$ & $1.3 \pm 0.6 \mathrm{E}-03$ & $\mathrm{I}$ \\
\hline g7 & 794899 & 0.6796 & -0.0376 & 7.33 & 20 & 7.5 & $9.4 \pm 1.2$ & $3.1 \pm 0.7 \mathrm{E}+03$ & $5.8 \pm 5.8 \mathrm{E}-04$ & I \\
\hline g9 & 789483 & 0.6667 & -0.0352 & 1.37 & 169 & 27.0 & $21.7 \pm 7.0$ & $6.1 \pm 6.4 \mathrm{E}+04$ & $1.6 \pm 1.5 \mathrm{E}-03$ & I \\
\hline $\mathrm{g} 10$ & 798750 & 0.6655 & -0.0535 & 1.96 & 12 & 5.1 & $9.5 \pm 0.7$ & $2.0 \pm 0.5 \mathrm{E}+03$ & $3.5 \pm 8.4 \mathrm{E}-04$ & I \\
\hline g16 & 639320 & 0.3763 & 0.0402 & 10.22 & 9 & 14.0 & $10.1 \pm 0.6$ & $3.2 \pm 0.6 \mathrm{E}+03$ & $8.1 \pm 2.7 \mathrm{E}-04$ & I \\
\hline g23 & 530763 & 359.9316 & -0.0626 & 2.72 & 32 & 17.9 & $12.0 \pm 3.2$ & $9.9 \pm 4.6 \mathrm{E}+03$ & $1.3 \pm 0.6 \mathrm{E}-03$ & I \\
\hline g25 & 506137 & 359.8412 & -0.0793 & 2.76 & 39 & 12.5 & $10.3 \pm 1.2$ & $5.5 \pm 1.9 \mathrm{E}+03$ & $3.1 \pm 1.8 \mathrm{E}-04$ & I \\
\hline g27 & 384307 & 359.5995 & -0.0316 & 4.77 & 3 & 35.0 & $17.5 \pm 3.0$ & $3.2 \pm 1.9 \mathrm{E}+04$ & $2.2 \pm 3.1 \mathrm{E}-03$ & II \\
\hline g29 & 366044 & 359.4362 & -0.1017 & 3.18 & 70 & 22.3 & $14.1 \pm 2.4$ & $1.9 \pm 0.9 \mathrm{E}+04$ & $1.1 \pm 0.4 \mathrm{E}-03$ & I \\
\hline g30 & 246410 & 359.3009 & 0.0334 & 1.87 & 11 & 9.8 & $8.9 \pm 0.7$ & $1.7 \pm 0.5 \mathrm{E}+03$ & $2.0 \pm 1.9 \mathrm{E}-04$ & I \\
\hline g31 & 214725 & 359.1994 & 0.0419 & 2.75 & 69 & 35.9 & $14.4 \pm 4.6$ & $2.3 \pm 1.6 \mathrm{E}+04$ & $0.0 \pm 0.0 \mathrm{E}-00$ & II \\
\hline g32 & 142942 & 358.9795 & 0.0840 & 0.00 & 374 & 10.9 & $10.5 \pm 6.0$ & $0.8 \pm 1.4 \mathrm{E}+04$ & $0.7 \pm 1.0 \mathrm{E}-03$ & $\mathrm{I}$ \\
\hline
\end{tabular}

neighborhood and the blue cross is that for the Galactic center. The black line has a slope of 1.4, corresponding to the SchmidtKennicutt relationship found for external galaxies (Kennicutt 1998). The agreement is excellent, suggesting that to first order the star formation in the Galactic center region is not strongly affected by the environmental differences with those in the disk of the Galaxy.

\section{CONCLUSIONS}

In this paper, we have studied the distribution of interstellar dust clouds and the nature of star formation in the Galactic center region using the $24 \mu \mathrm{m}$ Spitzer MIPS survey augmented with additional mid-IR, near-IR, submillimeter, and radio data. By making use of $M S X$ data in regions where the MIPS data are saturated, we constructed a complete $24 \mu \mathrm{m}$ image of the Galactic center region with unprecedented sensitivity and resolution.

The comparison between the $24 \mu \mathrm{m}$ and $8 \mu \mathrm{m}$ images showed numerous examples of compact regions of $24 \mu \mathrm{m}$ emission surrounded by partial shells of $8 \mu \mathrm{m}$ emission. These structures are characteristic of $\mathrm{H}$ II regions, as seen throughout the Galactic plane, where luminous stars have heated large dust grains thus enhancing $24 \mu \mathrm{m}$ emission, and have destroyed PAHs thus suppressing $8 \mu \mathrm{m}$ emission.

We noted a number of elongated IRDCs distributed over a wide range of angular scales toward the Galactic center. Many of the IRDCs lie within a "bow-tie" structure, consisting of two layers parallel to the Galactic equator and at both positive 

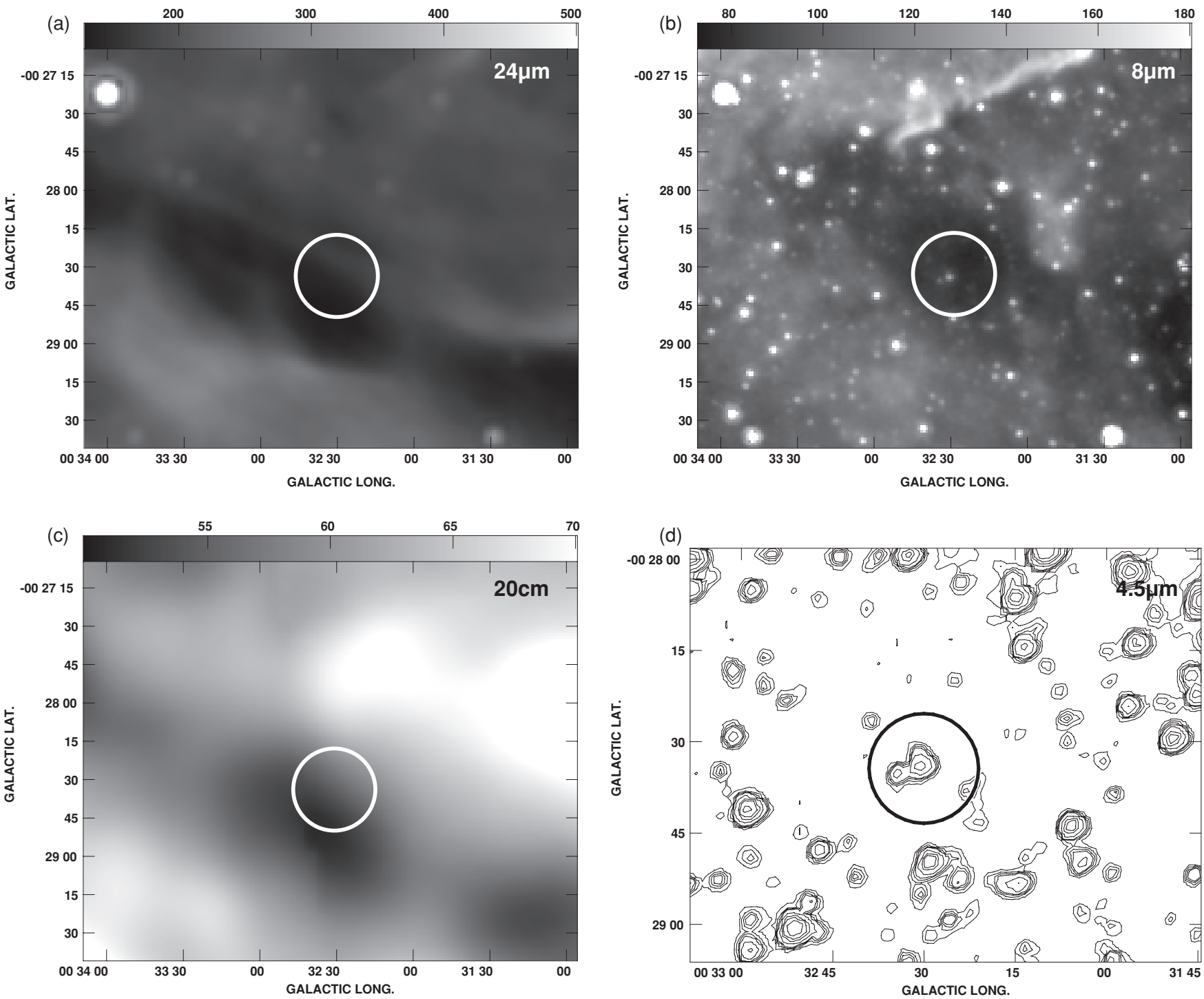

Figure 27. (a) Gray-scale image of g11 at $24 \mu \mathrm{m}$ with a flux range between 130 and $500 \mathrm{MJy} \mathrm{Sr}^{-1}$. (b) Similar to (a) except at $8 \mu \mathrm{m}$ with a flux range between 73 and $180 \mathrm{MJy} \mathrm{sr}^{-1}$. (c) A $20 \mathrm{~cm}$ continuum image with a flux range between 50 and $80 \mathrm{mJy}$ beam ${ }^{-1}$. (d) Contours of $4.5 \mu \mathrm{m}$ emission from g11 with contours at levels of 15, 20, 25, 30, 50, 100, 150, and $300 \mathrm{MJy} \mathrm{sr}^{-1}$. Symbols are as defined in Figure 17.

Table 10

The Parameters of SED Fits to $4.5 \mu \mathrm{m}$ Excess Sources Foreground to the Galactic Center

\begin{tabular}{|c|c|c|c|c|c|c|c|c|c|c|}
\hline Green No. & SST GC No. & $l$ & $b$ & $\chi_{\min }^{2}$ & nfits & $\left\langle A_{v}\right\rangle$ & $\left\langle M_{\star}\right\rangle$ & $\left\langle L_{\star}\right\rangle$ & $\left\langle\dot{M}_{\text {env }}\right\rangle$ & $\langle$ Stage $\rangle$ \\
\hline $\mathrm{g} 1$ & 1060982 & 0.9555 & -0.7853 & 0.96 & 12 & 5.6 & $2.4 \pm 0.6$ & $2.4 \pm 0.7 \mathrm{E}+01$ & $6.2 \pm 2.4 \mathrm{E}-06$ & I \\
\hline g2 & 1043897 & 0.8679 & -0.6963 & 1.97 & 547 & 33.1 & $5.5 \pm 1.3$ & $8.0 \pm 5.7 \mathrm{E}+02$ & $1.0 \pm 3.0 \mathrm{E}-05$ & I \\
\hline g4 & 1042278 & 0.7800 & -0.7395 & 0.77 & 161 & 10.3 & $6.9 \pm 1.9$ & $8.9 \pm 8.0 \mathrm{E}+02$ & $9.5 \pm 6.3 \mathrm{E}-05$ & I \\
\hline g5 & 532335 & 0.7062 & 0.4075 & 1.11 & 89 & 4.7 & $6.0 \pm 3.0$ & $1.7 \pm 4.4 \mathrm{E}+03$ & $1.2 \pm 1.4 \mathrm{E}-04$ & I \\
\hline g11 & 946558 & 0.5418 & -0.4759 & 0.25 & 152 & 5.0 & $5.2 \pm 2.5$ & $0.7 \pm 2.4 \mathrm{E}+03$ & $1.1 \pm 1.2 \mathrm{E}-04$ & I \\
\hline $\mathrm{g} 12$ & 992035 & 0.5172 & -0.6569 & 0.00 & 2643 & 9.9 & $2.1 \pm 1.2$ & $3.1 \pm 3.4 \mathrm{E}+01$ & $3.4 \pm 7.3 \mathrm{E}-05$ & I \\
\hline $\mathrm{g} 13$ & 997790 & 0.4835 & -0.7004 & 1.82 & 4 & 4.7 & $9.9 \pm 1.7$ & $3.3 \pm 2.5 \mathrm{E}+03$ & $0.6 \pm 1.0 \mathrm{E}-03$ & I \\
\hline g14 & 1003258 & 0.4771 & -0.7268 & 1.69 & 24 & 4.9 & $7.4 \pm 1.7$ & $1.0 \pm 1.3 \mathrm{E}+03$ & $2.7 \pm 1.4 \mathrm{E}-04$ & I \\
\hline $\mathrm{g} 15$ & 929122 & 0.4084 & -0.5042 & 0.31 & 45 & 10.3 & $14.3 \pm 4.2$ & $1.3 \pm 0.8 \mathrm{E}+03$ & $1.0 \pm 0.4 \mathrm{E}-03$ & I \\
\hline $\mathrm{g} 17$ & 764611 & 0.3153 & -0.2010 & 0.66 & 75 & 11.6 & $12.7 \pm 2.6$ & $1.0 \pm 0.2 \mathrm{E}+04$ & $1.0 \pm 2.8 \mathrm{E}-04$ & I \\
\hline $\mathrm{g} 18$ & 842427 & 0.1667 & -0.4455 & 1.15 & 43 & 19.5 & $14.0 \pm 4.3$ & $1.7 \pm 1.0 \mathrm{E}+04$ & $2.1 \pm 1.2 \mathrm{E}-03$ & I \\
\hline g19 & 916902 & 0.0915 & -0.6624 & 6.10 & 45 & 4.4 & $5.5 \pm 1.3$ & $2.7 \pm 1.9 \mathrm{E}+02$ & $1.5 \pm 1.4 \mathrm{E}-04$ & I \\
\hline $\mathrm{g} 20$ & 906570 & 0.0842 & -0.6415 & 4.54 & 33 & 15.2 & $7.3 \pm 1.3$ & $9.3 \pm 7.7 \mathrm{E}+02$ & $2.8 \pm 2.0 \mathrm{E}-04$ & I \\
\hline $\mathrm{g} 21 \mathrm{~B}$ & 789457 & 359.9695 & -0.4573 & 0.38 & 135 & 23.7 & $23.8 \pm 6.6$ & $4.4 \pm 5.5 \mathrm{E}+04$ & $0.5 \pm 1.1 \mathrm{E}-03$ & I \\
\hline $\mathrm{g} 21 \mathrm{~A}$ & 788629 & 359.9700 & -0.4554 & 0.73 & 7648 & 13.9 & $11.8 \pm 2.5$ & $1.1 \pm 0.7 \mathrm{E}+04$ & $0.8 \pm 3.1 \mathrm{E}-04$ & I \\
\hline $\mathrm{g} 22$ & 387542 & 359.9386 & 0.1709 & 0.01 & 396 & 8.3 & $4.9 \pm 1.8$ & $1.8 \pm 0.9 \mathrm{E}+03$ & $3.2 \pm 5.2 \mathrm{E}-04$ & I \\
\hline g24 & 676294 & 359.9072 & -0.3026 & 0.44 & 50 & 8.6 & $6.3 \pm 2.5$ & $2.0 \pm 0.7 \mathrm{E}+03$ & $3.3 \pm 2.4 \mathrm{E}-04$ & I \\
\hline g26 & 523105 & 359.6148 & -0.2438 & 3.63 & 3447 & 0.0 & $7.1 \pm 0.9$ & $0.0 \pm 0.0 \mathrm{E}+00$ & $0.0 \pm 0.0 \mathrm{E}+00$ & \\
\hline $\mathrm{g} 28$ & 214738 & 359.5701 & 0.2704 & 1.16 & 148 & 4.2 & $4.0 \pm 1.3$ & $1.1 \pm 0.8 \mathrm{E}+02$ & $6.0 \pm 6.0 \mathrm{E}-05$ & I \\
\hline
\end{tabular}



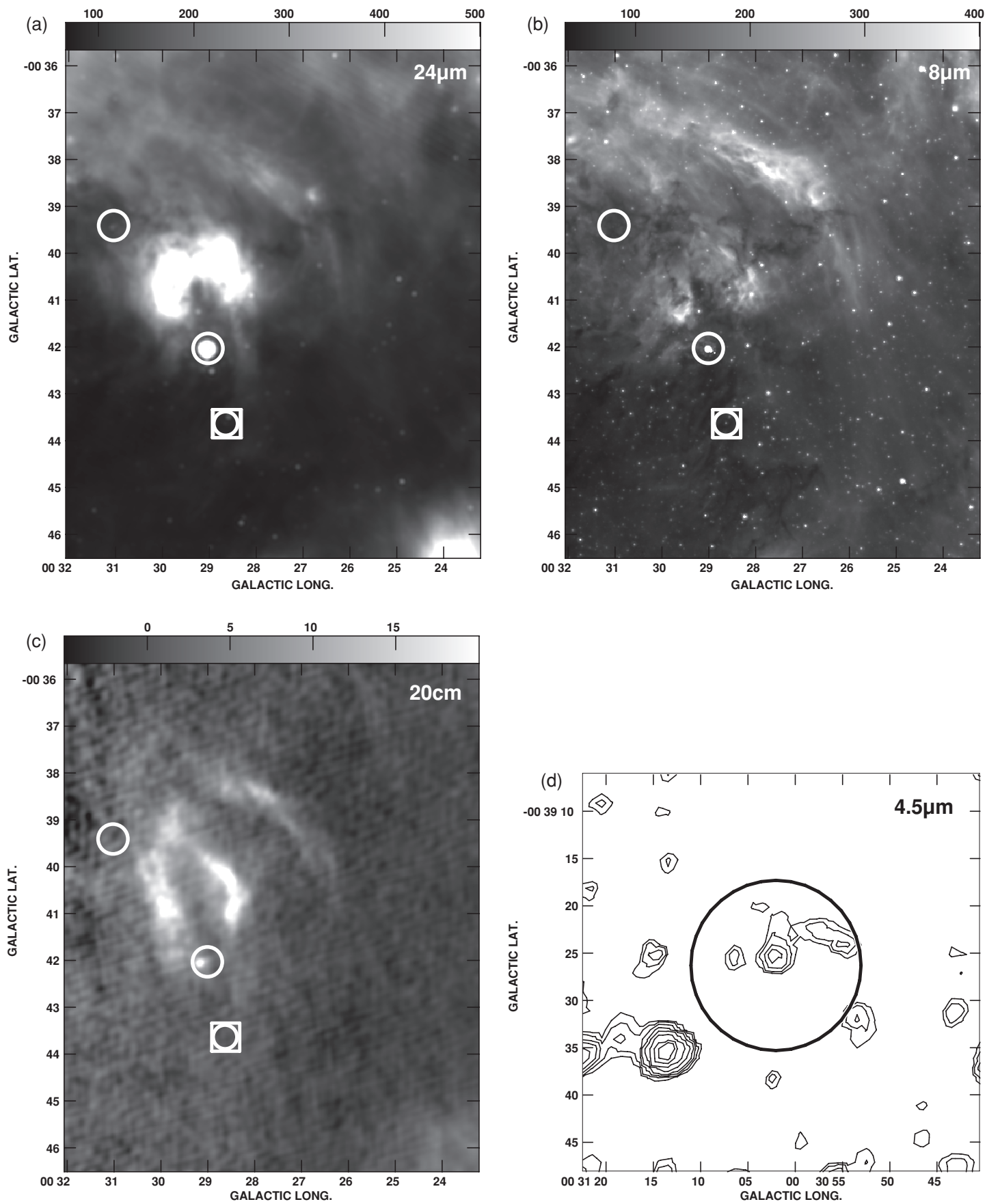

Figure 28. (a) Gray-scale image of g12, g13,g14 at $24 \mu \mathrm{m}$ with a flux range between 66 and $500 \mathrm{MJy} \mathrm{sr}^{-1}$. (b) Similar to (a) except at $8 \mu \mathrm{m}$ with a flux range between 39 and $400 \mathrm{MJy} \mathrm{sr}^{-1}$. (c) Similar to (a) except at $20 \mathrm{~cm}$ with a flux range between -5 and $20 \mathrm{mJy}^{\mathrm{beam}}{ }^{-1}$. (d) Contours of $4.5 \mu \mathrm{m}$ emission from g12 at $9,11,15$, $20,30,50,100,150,300$, and $500 \mathrm{MJy} \mathrm{sr}^{-1}$. Symbols are as defined in Figure 17. (e) Contours of $4.5 \mu \mathrm{m}$ emission from g13 with the same levels as those of (d). (f) Contours of $4.5 \mu \mathrm{m}$ emission from g14 with the same levels as those of (d). Symbols are as defined in Figure 17.

and negative longitudes. Molecular line survey studies show counterparts to a large fraction of the IRDC of the bowtie structure. The darkness of the two coherent IRDC layers provides relative locations of individual clouds with respect to each other. In particular, we note that clouds distributed on the positive longitudes (e.g., Sgr B2 and G0.25+0.01) are closer to us than those on the negative longitudes.

A result of the $24 \mu \mathrm{m}$ imaging is the recognition of a population of compact and point sources distributed between the two layers of IRDCs. This population is predominantly found at negative galactic longitudes, and includes a distinct cluster of sources at G359.43+0.02. Many of these sources are YSO candidates. Candidates are selected as particularly red objects in the [8]-[24] $\mu \mathrm{m}$ CMD, and characterized by modeling their SEDs. This led us to estimate the SFR of $0.14 M_{\odot} \mathrm{yr}^{-1}$ based on SED fitted Stage I YSO candidates. The preponderance of Stage I YSO candidates implies that massive star formation took place in the nuclear disk about $10^{5} \mathrm{yr}$ ago. The derived scale heights of the population of YSO candidates ( $h \sim 7 \mathrm{pc}$ ) as well as that of the molecular layer $(h \sim 10 \mathrm{pc})$ suggest their being dynamically young. These measurements are consistent with a highly efficient burst of high-mass star formation in the nuclear disk about $10^{5} \mathrm{yr}$ ago. This aspect of the study characterizes the unique nature of the Galactic center region, 

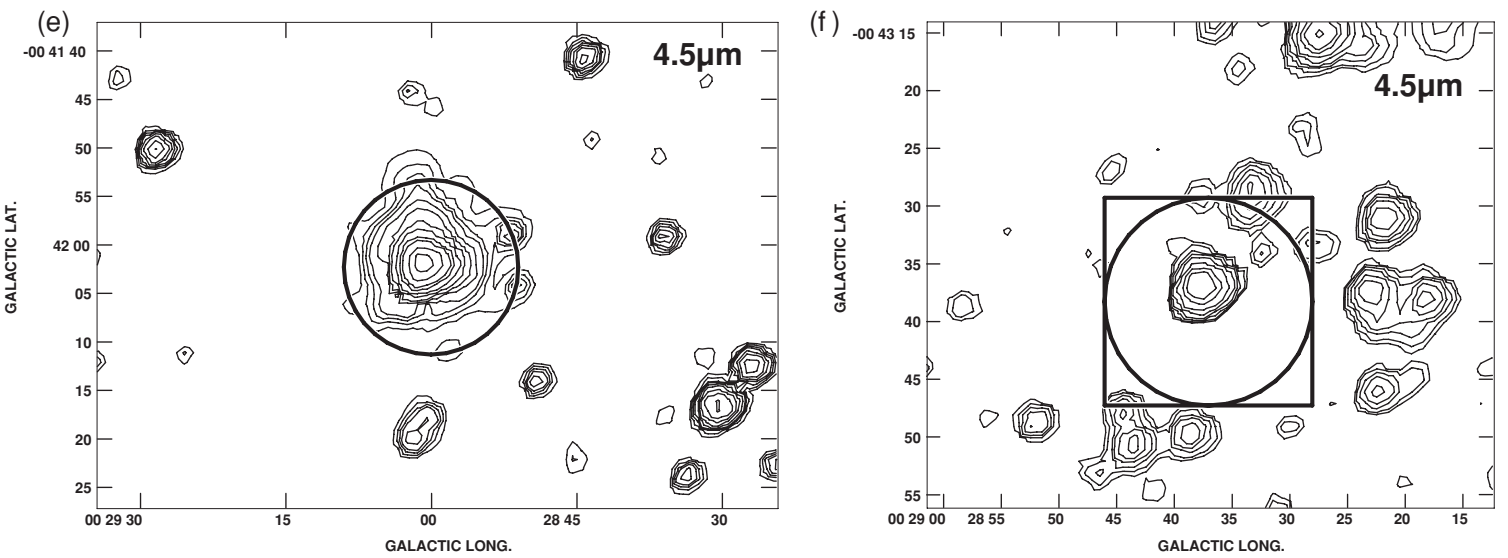

Figure 28. (Continued)
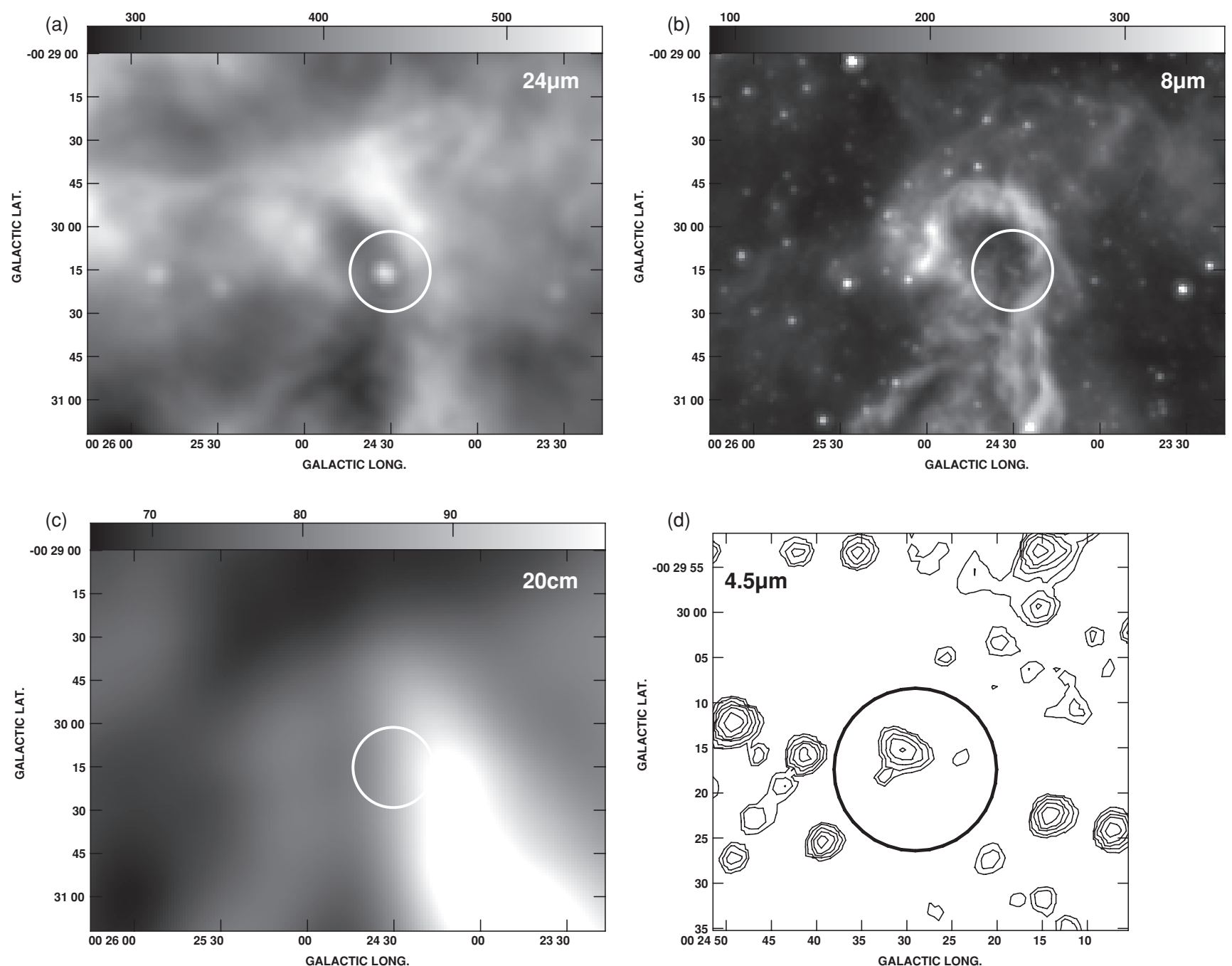

Figure 29. (a) Gray-scale image of g15 at $24 \mu \mathrm{m}$ with a flux range between 272 and $551 \mathrm{MJy} \mathrm{sr}^{-1}$. (b) Similar to (a) except at $8 \mu \mathrm{m}$ with a flux range between 88 and $350 \mathrm{MJy} \mathrm{sr}^{-1}$. (c) A $20 \mathrm{~cm}$ continuum image with a flux range between 66 and $100 \mathrm{mJy}$ beam $^{-1}$. (d) Contours of $4.5 \mu \mathrm{m}$ emission from g15 with contours at levels of $9,11,15,20,30,50,100,150,300$, and $500 \mathrm{MJy} \mathrm{sr}^{-1}$. Symbols are as defined in Figure 17.

apart from the general Galactic disk. Evidence for large-scale IRDCs throughout the Galactic center is consistent with the idea that massive star formation and massive cluster formation take place in the central region of the Galaxy. Future study of this region with its steep gravitational potential provides a testing ground for large-scale formation of massive stars and/or star clusters.
Finally, we explored an independent means of identifying YSO candidates by finding sources with excess $4.5 \mu \mathrm{m}$ emission, indicating the presence of shocked molecular emission from protostellar sources. This color distinction was used as a proxy to probe the sites of star formation even earlier than $10^{5} \mathrm{yr}$ old. We found 33 sources that show excess $4.5 \mu \mathrm{m}$ emission. Those at the lowest latitudes were presumed to be at the 

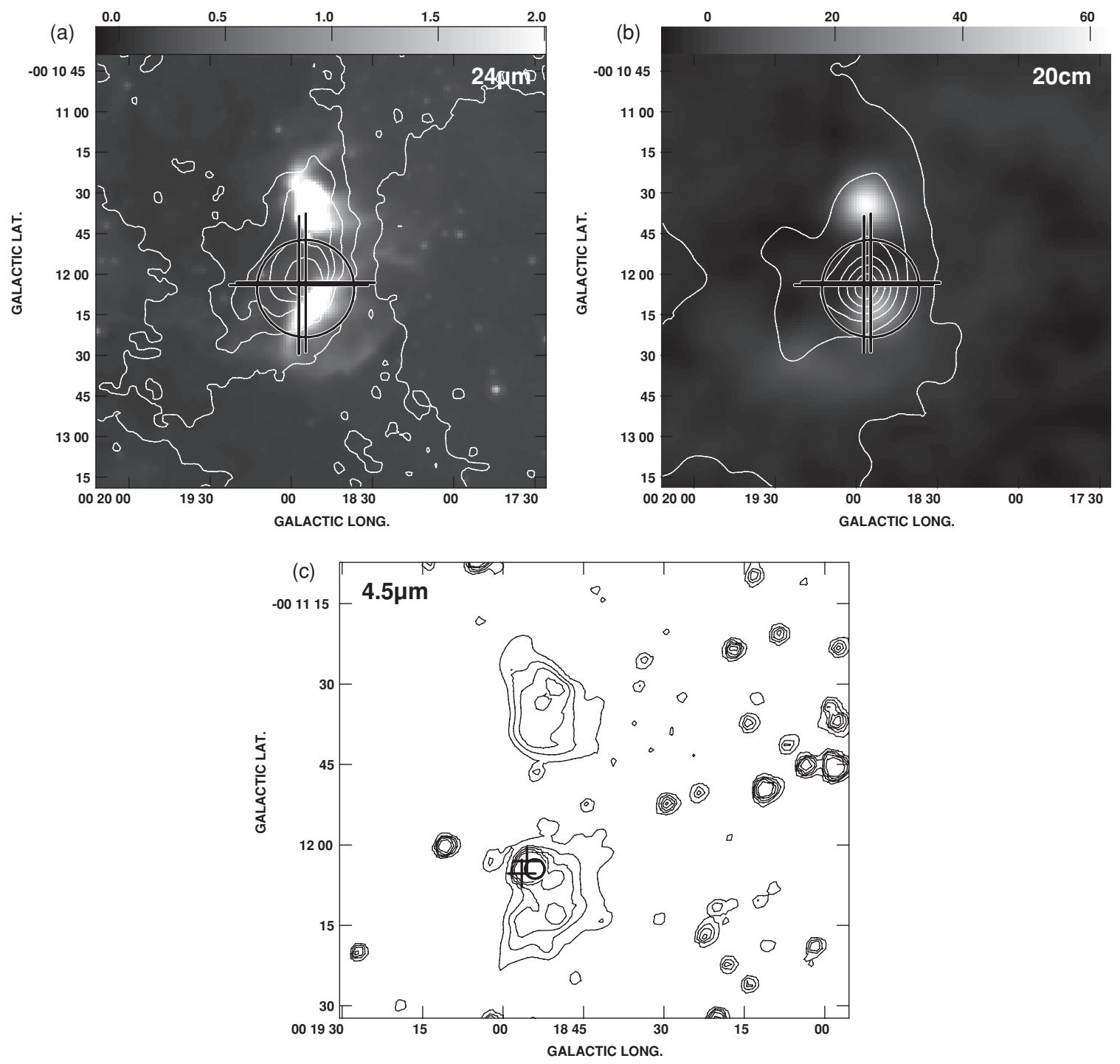

Figure 30. (a) Contours of $450 \mu \mathrm{m}$ emission from g 17 at $8,10,13,16,19,25 \mathrm{Jy}_{\text {beam }}{ }^{-1}$ are superimposed on a $8 \mu \mathrm{m}$ image. (b) Contours of $850 \mu \mathrm{m}$ emission from g17 at $0.5,1,2,3,4,5,6,7 \mathrm{Jy}_{\text {beam }}^{-1}$ are superimposed on a $20 \mathrm{~cm}$ continuum image. (c) Contours of $4.5 \mu \mathrm{m}$ emission from g17 at levels of 60, 100, 140, 200, and $300 \mathrm{MJy} \mathrm{sr}^{-1}$. Symbols are as defined in Figure 17.

Galactic center, while those at higher latitudes often shown indication of being more local sources. SED fitting as well as the correlation of $4.5 \mu \mathrm{m}$ excess sources with methanol masers and IRDCs indicated the majority of these YSO candidates in Stage I and are likely to be associated with molecular outflows in sites of on-going star formation. Assuming that the $4.5 \mu \mathrm{m}$ excess sources provide us with all the sites of on-going star formation and that the SED fitted mass determination of the $4.5 \mu \mathrm{m}$ excess sources is applicable to these protostars, then we conclude that the efficiency of on-going star formation is lower than that estimated for the Galactic disk and is consistent with earlier studies of this region (Lis \& Carlstrom 1994). The estimated values of SFR over different time scales up to $10^{7} \mathrm{yr}$ ago suggest that large-scale star formation in the nuclear disk is likely to be continuous over a long period. However, mild burst-like activity could increase SFR by an order of magnitude on a $10^{5} \mathrm{yr}$ timescale.

We acknowledge the use of SCUBA data taken by D. PiercePrice. This work is partially supported by NSF under award number AST-0807400 and from JPL/Caltech contract 1255094.

\section{APPENDIX}

We assumed that the sources that are distributed at high galactic latitudes are most likely foreground sources whereas the low latitude sources $\left(|b|<10^{\prime}\right)$ are near the Galactic center. We first describe $4.5 \mu \mathrm{m}$ excesses sources which are most likely located in the Galactic center region followed by foreground sources. In all the figures presented below, the circles mark the position of the $4.5 \mu \mathrm{m}$ excess sources. For extended $4.5 \mu \mathrm{m}$ sources, the peak of the excess emission is used to identify the closest point sources in the IRAC and MIPS catalogs by Ramirez et al. (2008) and Hinz et al. (2009), respectively.

The squares represent sources that were observed at $70 \mu \mathrm{m}$. The distribution of class I and II methanol masers are also drawn on these figures as plus $(+)$ and cross $(\times)$ signs, respectively.

\section{A.1. Galactic Center $4.5 \mu \mathrm{m}$ Excess Sources}

G1.041-0.072 ( $g 0)$. This source lies at the eastern edge of the ridge of IRDCs and is not well fit as it shows a narrow peak in its SED, thus it is unlikely to be at an early phase of a YSO. The fitted SED gives a protostellar source with a mass of $\sim 10 M_{\odot}$. 
This $4.5 \mu \mathrm{m}$ excess source has a $24 \mu \mathrm{m}$ counterpart, as shown in Figure 17(a) and lies within an arcminute of an extended $8 \mu \mathrm{m}$ and $24 \mu \mathrm{m}$ H II source G1.05-0.071 at $l=1^{\circ} 3^{\prime} 4^{\prime \prime}, b=-4^{\prime} 15^{\prime \prime}$. This extended source is also shown in the three-color image, as presented in Figure 13. Figure 17(b) shows contours of $850 \mu \mathrm{m}$ emission that trace the elongated east-west IRDC. The strongest $850 \mu \mathrm{m}$ emission coincides with G1.05-0.071.

G0.826-0.211 $(\mathrm{g} 3)$. This $4.5 \mu \mathrm{m}$ excess source is embedded in the IRDC associated with Sgr B2. The distribution of the IRDC ridge at the location of $\mathrm{g} 3$ widens in a plume-like structure pointed southward of the Galactic plane. Figures $17(\mathrm{c})$ and (d) show contours of 450 and $850 \mu \mathrm{m}$ emission from this prominent IRDC. A $24 \mu \mathrm{m}$ and $8 \mu \mathrm{m}$ source coincides with $\mathrm{g} 3$, which is shown as a circle in Figure 17. This $4.5 \mu \mathrm{m}$ excess source was also observed at $70 \mu \mathrm{m}$, as shown by a square sign in Figures 17(c) and (d), but was not detected (see Table 6). Most of the well known ultracompact $\mathrm{H}$ II regions associated with $\mathrm{Sgr}$ B2 are located to the northwest of g3, as shown in Figure 14(a). The SED fit to this source gives a mass of $13.7 M_{\odot}$. This $4.5 \mu \mathrm{m}$ excess source is located within $20^{\prime \prime}$ of $\mathrm{SiO} 17452$ 2819 with a radial velocity of $90 \mathrm{~km} \mathrm{~s}^{-1}$ (Shiki et al. 1997). The location of this maser source with respect to $\mathrm{g} 3$ supports the idea that this $\mathrm{SiO}$ maser is associated with the Sgr B2 starforming region and is a counterpart to a YSO. The correlation of submillimeter emission, IRDC, $24 \mu \mathrm{m}$ point source and excess $4.5 \mu \mathrm{m}$ emission is consistent with $\mathrm{g} 3$ being a massive YSO associated with an active core.

Sgr B2 ( $g 6-g 10)$. The largest concentration of $4.5 \mu \mathrm{m}$ excess sources is located in Sgr B2, which is one of the most massive star-forming sites in the Galaxy. SED fits to the five $4.5 \mu \mathrm{m}$ excess sources g6 to g10 give the highest protostellar masses among the $4.5 \mu \mathrm{m}$ excess sources, ranging between 8 and $22 M_{\odot}$. Figures $18(\mathrm{a})$ and (b) show contours of 450 and $850 \mu \mathrm{m}$ emission that are superimposed on $8 \mu \mathrm{m}$ and $20 \mathrm{~cm}$ images of Sgr B2, respectively. It is clear that the IRDC associated with Sgr B2 is traced remarkably well by submillimeter and molecular line emission from several active molecular cores (Jones et al. 2008). The distribution of compact H II regions, as shown best at $20 \mathrm{~cm}$ wavelength, indicates that they lie at the southern edge of the dark cloud. The distribution of UC $\mathrm{H}$ II regions as well as $24 \mu \mathrm{m}$ sources suggests a later phase of massive star formation at the southern edge of IRDC where UC H II regions are concentrated. However, the distribution of methanol sources is seen silhouetted against the middle of the IRDC. Figure 18(c) makes this point even clearer by showing that most of the methanol sources are displaced with respect to $24 \mu \mathrm{m}$ sources heated by UC H II regions lie at the edge of the IRDC. The high column density of Sgr B2 and the saturation of the brighter $24 \mu \mathrm{m}$ sources are likely responsible for a lack of additional detection of $4.5 \mu \mathrm{m}$ excess sources and YSO candidates in Sgr B2. Nevertheless, the projected displacement between $24 \mu \mathrm{m}$ sources and methanol sources is consistent with a picture that star formation in the IRDC associated with Sgr B2 has taken place from outside in.

Figure 18(d) shows contours of emission from one of the $4.5 \mu \mathrm{m}$ excess sources, g6, that shows extended emission at $4.5 \mu \mathrm{m}$. Three of the five $4.5 \mu \mathrm{m}$ excess sources, g6, g8, and g9, coincide with ultracompact $\mathrm{H}$ II regions $\mathrm{Sgr}$ B2 R, I, and F (Gaume et al. 1995). We note that Sgr B2-F itself breaks up into multiple radio components that are considered to be members of a cluster of massive ultracompact $\mathrm{H}$ II regions (De Pree et al. 2005). The parameters of the SED fit to g9 should be considered with caution, given that multiple sources could
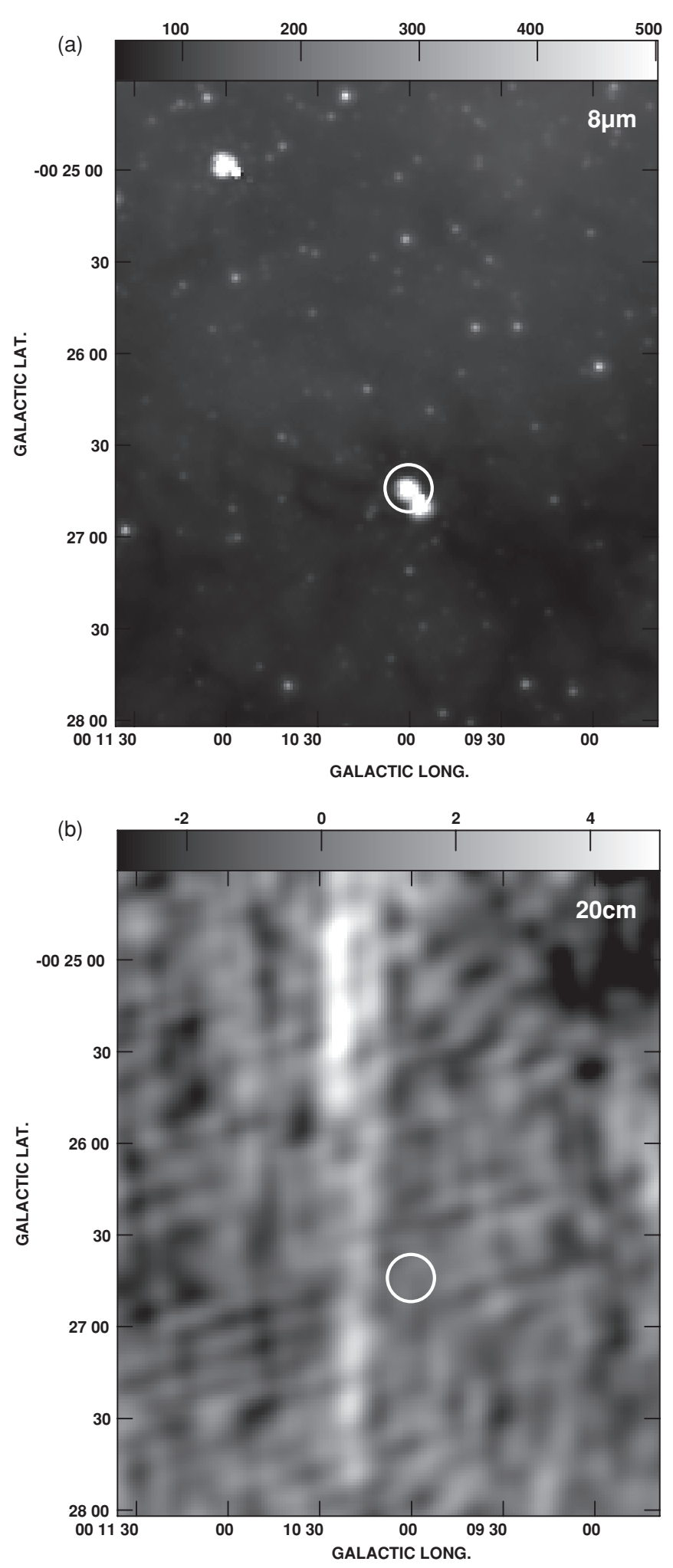

Figure 31. (a) Gray-scale $8 \mu \mathrm{m}$ image of the region toward g18 with flux range between 45 and $500 \mathrm{MJy} \mathrm{sr}^{-1}$ (b) Gray-scale $20 \mathrm{~cm}$ continuum image toward $\mathrm{g} 18$ with flux range between -3 and $5 \mathrm{mJy}$ beam $^{-1}$. Symbols are as defined in Figure 17.

contribute to the SED. Fortunately, the flux from a cluster of massive stars is likely to be dominated by the most massive star as luminosity is a steep power of mass and thus the assumption of all the luminosity arising from a single cluster member is reasonable. 
$G 0.376+0.04(\mathrm{~g} 16)$. The $4.5 \mu \mathrm{m}$ excess source associated with g16 is detected against the IRDC ridge at G0.376+0.04. This source coincides with one of the string of submillimeter emitting clouds, known as the dust ridge in Figure 1(c) (Lis \& Menten 1998). g16 coincides with a class II methanol maser at a velocity of $37 \mathrm{~km} \mathrm{~s}^{-1}$ that is listed as source 8 in Table 8 . Molecular line studies of the ridge of IRDCs are dominated by velocities ranging between 20 and $30 \mathrm{~km} \mathrm{~s}^{-1}$ (Oka et al. 2005). Figures 19(a) and (b) show contours of $450 \mu \mathrm{m}$ and $850 \mu \mathrm{m}$ emission and are superimposed on gray-scale $24 \mu \mathrm{m}$ and $20 \mathrm{~cm}$ images of this cloud. This is the first evidence that this cloud shows a signature of massive star formation as the fitted SED indicates $10 M_{\odot}$ for this YSO, the first evidence that this cloud is a site of massive star formation (Argon et al. 2000). Following the definition of active and quiescent cores by Chambers et al. (2009), the eastern half of the IRDC ridge is in its quiescent phase of star formation whereas the western half is forming massive YSOs. The $4.5 \mu \mathrm{m}$ excess source also lies in the vicinity of a nonthermal radio filament that appears to terminate in the IRDC and about $1^{\prime}$ east of g16, as shown in Figure 19(b). Figure 19(c) shows contours of $4.5 \mu \mathrm{m}$ emission from $\mathrm{G} 0.376+0.04$.

G359.932-0.063 (g23). This $4.5 \mu \mathrm{m}$ excess source lies at the edge of the $50 \mathrm{~km} \mathrm{~s}^{-1}$ molecular cloud M-0.13-0.08 (Herrnstein \& Ho 2005; Armstrong \& Barrett 1985). Figures 20(a) and (b) show contours of submillimeter emission from two prominent $50 \mathrm{~km} \mathrm{~s}^{-1}$ and $20 \mathrm{~km} \mathrm{~s}^{-1}$ molecular clouds, known to be the closest clouds to the Galactic center. Contours of $450 \mu \mathrm{m}$ and $850 \mu \mathrm{m}$ emission from these clouds are superimposed on the $8 \mu \mathrm{m}$ and $20 \mathrm{~cm}$ images of the Sgr A region, respectively. The $50 \mathrm{~km} \mathrm{~s}^{-1}$ cloud is known to be interacting with the Sgr A East SNR G0.0+0.0 (Zylka et al. 1990) and several OH masers at $1720 \mathrm{MHz}$ have trace the interaction site (YusefZadeh et al. 1999). The shell-like Sgr A East SNR is shown in Figure 20(b). The position of g23 lies within 5 arcsec of $\mathrm{OH}(1720 \mathrm{MHz})$ maser $\mathrm{H}$ at a velocity of $49 \mathrm{~km} \mathrm{~s}^{-1}$. The excess $4.5 \mu \mathrm{m}$ emission at this position could be associated with the supernova shock interaction. $4.5 \mu \mathrm{m}$ excess emission has been seen toward a number of SNRs (Reach et al. 2006). However, the parameters of the SED of this source suggest that G359.23-0.063 is associated with a $11 M_{\odot}$ protostar (see Table 9). If so, the protostar presents the earliest phase of massive star formation in the $50 \mathrm{~km} \mathrm{~s}^{-1}$ cloud, perhaps triggered by the interaction with the expanding Sgr A East SNR. A chain of ultracompact $\mathrm{H}_{\mathrm{II}}$ regions $\sim 2^{\prime}$ northeast of g23 may trace massive star formation at a later phase than that shown by g23. Figure 20(c) shows a $24 \mu \mathrm{m}$ image of IRDC associated with the $50 \mathrm{~km} \mathrm{~s}^{-1}$ and clearly shows the IRDC toward the region where g23 is detected. The evidence for collisionally excited methanol emission has also been reported toward the $50 \mathrm{~km} \mathrm{~s}^{-1}$ (Haschick et al. 1990; see source 2 in Table 7). This result supports the presence of massive star formation in this region. Figure 20(d) shows contours of $4.5 \mu \mathrm{m}$ emission from $\mathrm{g} 23$.

The $20 \mathrm{~km} \mathrm{~s}^{-1}$ and $50 \mathrm{~km} \mathrm{~s}^{-1}$ IRDCs are also located in a region where 19 radio filaments (A1-A19) have been detected at $20 \mathrm{~cm}$ (Yusef-Zadeh et al. 2004). Two of these nonthermal radio filaments RF-A3 and RF-A9 are labeled in Figure 20(b). The nonthermal filaments (e.g., G359.90-0.07 and G359.88-0.07) may be interacting with the $20 \mathrm{~km} \mathrm{~s}^{-1}$ molecular cloud (YusefZadeh et al. 2005).

G359.841-0.080 (g25). G359.841-0.08 is another $4.5 \mu \mathrm{m}$ excess source that is found at the western edge of the $20 \mathrm{~km} \mathrm{~s}^{-1}$ molecular cloud M-0.02-0.07 (Herrnstein \& Ho 2005; Armstrong \& Barrett 1985), which is traced by the prominent IRDC at $24 \mu \mathrm{m}$, as presented in Figure 20(c). Evidence for a signature of star formation is supported by the presence of star formation by a class I methanol maser and a $\mathrm{H}$ II region toward the eastern half of the cloud. The methanol maser source is detected at a velocity of $21 \mathrm{~km} \mathrm{~s}^{-1}$ (Haschick et al. 1990; Val'tts et al. 2000). Additional observations are needed to confirm the association of this $4.5 \mu \mathrm{m}$ excess source with the $20 \mathrm{~km} \mathrm{~s}^{-1}$ cloud. Contours of $4.5 \mu \mathrm{m}$ emission are shown in Figure 20(d). The peak of submillimeter emission from the $20 \mathrm{~km} \mathrm{~s}^{-1}$ IRDC shows no signs of star formation, thus, is considered to be a quiescent core.

G359.599-0.032 (g27). This $4.5 \mu \mathrm{m}$ excess source is projected against the IRDC ridge, on the negative longitude side of the Galactic center. Figures 21(a) and (b) show a $24 \mu \mathrm{m}$ and $6 \mathrm{~cm}$ continuum images of this source, respectively, and the contours of $4.5 \mu \mathrm{m}$ emission are shown in Figure 21(c). The parameters of the fit to this source suggests a $14.5 \pm 3.5 M_{\odot}$ protostar. However, g27 shows a narrow peak in its SED and is not fitted well by the models. This source is located about $35^{\prime \prime}$ north of an $\mathrm{H}$ II region and a shell-like structure with a diameter of $30^{\prime \prime}$ centered at G359.61-0.045. The shell-like structure is best seen at $24 \mu \mathrm{m}$ in Figure 21(a).

G359.437-0.102 (g29). Figures 22(a) and (b) show contours of $450 \mu \mathrm{m}$ and $850 \mu \mathrm{m}$ emission superimposed on $8 \mu \mathrm{m}$ and $20 \mathrm{~cm}$ continuum images of the Sgr C H II region, respectively. This well known H II region is one of the brightest extended $\mathrm{H}$ II regions in the Galactic center region. The Sgr C H II region was saturated at $24 \mu \mathrm{m}$, so we combined the image with the MSX image at the same wavelength band. The Sgr $\mathrm{C}$ IRDC is elongated and runs roughly along the Galactic plane. It is detected at both $8 \mu \mathrm{m}$ and $24 \mu \mathrm{m}$, as shown in Figure 22(c). This IRDC is traced by submillimeter emission showing several cores along its elongated structure. The peak emission at $24 \mu \mathrm{m}$ coincides with the peak $\mathrm{H}$ II emission at $20 \mathrm{~cm}$ and suggests that warm grains are being heated by the central O4 star.

The $4.5 \mu \mathrm{m}$ excess source g29 lies at the peak of the submillimeter emission and is shown as a circle in Figures 22(a)(c). The crosses show the positions of class II methanol masers with velocities of -52 and $-53 \mathrm{~km} \mathrm{~s}^{-1}$. The masers are listed as sources number 1 and 2 in Table 8. Radio recombination line measurements of the Sgr C H II region show a radial velocity of $\sim-65 \mathrm{~km} \mathrm{~s}^{-1}$ (Liszt 1992; Liszt \& Spiker 1995). Figure 22(d) shows contours of the extended $4.5 \mu \mathrm{m}$ excess emission. The corresponding color image of the $4.5 \mu \mathrm{m}$ excess source can be seen in Figure 13. The modeled SED of the $4.5 \mu \mathrm{m}$ excess source G359.436-0.102 in Sgr C indicates that the central protostellar candidate coincides with the northern maser source. Figure 15(a) shows the best-fitted models to the data using 2MASS, IRAC, MIPS, and SCUBA data accounting for 22 mag of visual extinction. This SED fit is consistent with the identification of the $4.5 \mu \mathrm{m}$ excess source with a massive $14 M_{\odot}$ protostellar source (see Table 9) associated with the methanol masers.

The region surrounding $4.5 \mu \mathrm{m}$ excess source (g29) is complex. The emission at 4.5 and $5.8 \mu \mathrm{m}$ is extended whereas the emission at $8 \mu \mathrm{m}$ has a point-like structure and does not appear to have a counterpart at shorter wavelengths. The $8 \mu \mathrm{m}$ source is $\sim 6^{\prime \prime}$ away from the center of the $4.5 \mu \mathrm{m}$ emission. There are three other nearby compact sources; higher resolution study is needed to clarify the nature of these $8 \mu \mathrm{m}$ sources. However, it is most likely that the extended emission from the 

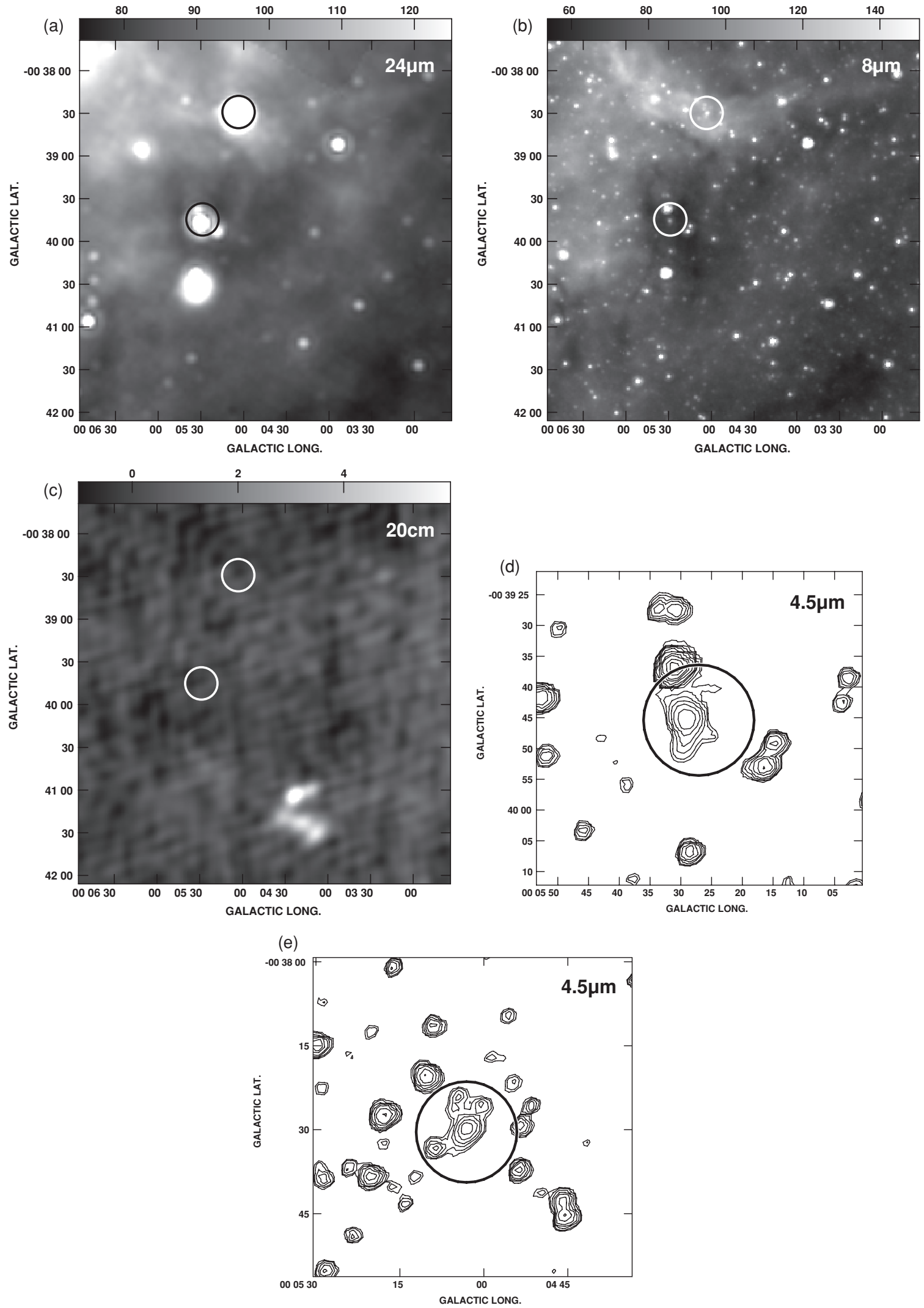

Figure 32. (a) Gray-scale $24 \mu \mathrm{m}$ image of g19 and g20 with flux range between 74 and $125 \mathrm{MJy} \mathrm{sr}^{-1}$. (b) Gray-scale $8 \mu \mathrm{m}$ image of g19 and g20 with flux range between 54 and $150 \mathrm{MJy} \mathrm{sr}^{-1}$. (c) Gray-scale $20 \mathrm{~cm}$ continuum image of g19 and g20 with flux range between -1 and $6 \mathrm{mJy}$ beam ${ }^{-1}$. (d) Contours of $4.5 \mu \mathrm{m}$ emission from g19 at 18, 22, 30, 40, 60, 100, 200, 300, 600, and $1000 \mathrm{MJy} \mathrm{sr}^{-1}$. (e) Contours of $4.5 \mu \mathrm{m}$ emission from g20 at 18, 22, 30, 40, 60, 100, 200, 300, 600, and $1000 \mathrm{MJy} \mathrm{s}^{-1}$. Symbols are as defined in Figure 17. 

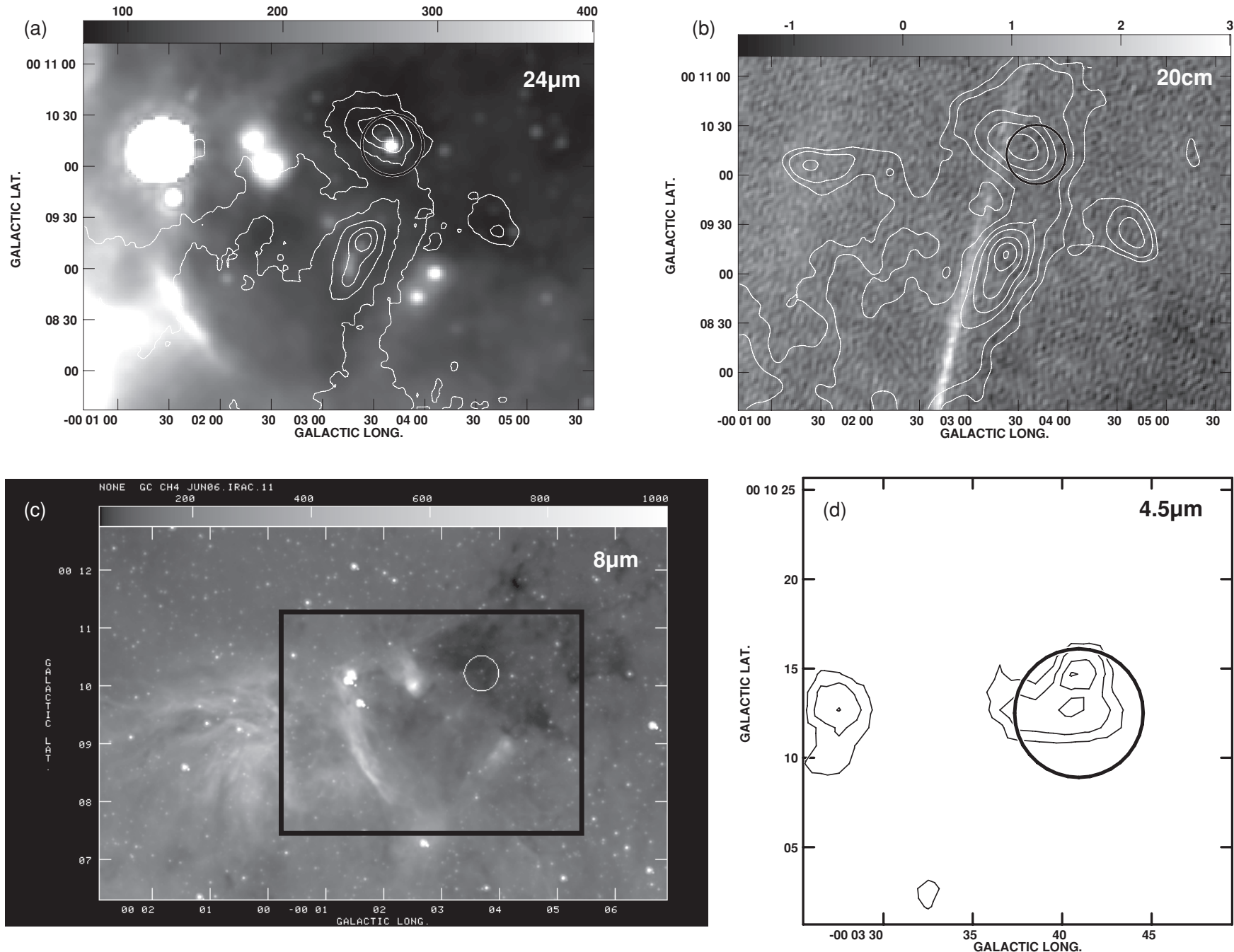

Figure 33. (a) Contours of $450 \mu \mathrm{m}$ emission from g22 at 2.5, 3, 3.5, 4, 5, 6, and $7 \mathrm{Jy}$ beam ${ }^{-1}$ are superimposed on a $24 \mu \mathrm{m}$ continuum image. (b) Contours of $850 \mu \mathrm{m}$ emission from g22 at $2.5,3,3.5,4,5,6$, and $7 \mathrm{Jy}$ beam ${ }^{-1}$ are superimposed on a $20 \mathrm{~cm}$ continuum image. (c) Gray-scale $8 \mu \mathrm{m}$ image of a large region presenting the distribution of PAH emission in the vicinity of g22. The rectangular box drawn on this figure corresponds to the area shown in (a) and (b). (d) Contours of $4.5 \mu \mathrm{m}$ emission from g22 is shown at 15, 20, 30, 40, 50, $60 \mathrm{MJy} \mathrm{sr}^{-1}$. Symbols are as defined in Figure 17.

region close to the $4.5 \mu \mathrm{m}$ excess source represents shocked molecular outflow from the YSO candidate. This is consistent with the presence of submillimeter core, methanol masers, IRDC, and $4.5 \mu \mathrm{m}$ excess, all of which suggest an active phase of massive star formation at the northwestern edge of Sgr C. The southeastern half of the IRDC shows no signs of active star formation.

There is a puzzling feature at the northeastern boundary of Sgr C IRDC, a lack of strong free-free radio continuum and submillimeter emission $l=-34^{\prime} 10^{\prime \prime}, b=-5^{\prime} 45^{\prime \prime}$. This implies that there is a cavity in the ionized gas and emission by dust at the interface between the submillimeter core and the Sgr C H II region. However, it is possible that the appearance of the cavity results from poor sensitivity in the submillimeter.

At $20 \mathrm{~cm}$, there is a compact bright continuum source at $l=-34^{\prime} 29^{\prime} .19, b=-6^{\prime} 38^{\prime \prime} 15$ which is about $1^{\prime}$ or $2.4 \mathrm{pc}$ (the Galactic center distance of $8.5 \mathrm{kpc}$ ) from the positions of the methanol masers. The flux density of this source is $20 \pm$ $1.8 \mathrm{mJy}$. The most prominent nonthermal radio filaments of Sgr $\mathrm{C}$, as shown in Figure 22(b), becomes quite faint inside the H II complex. The long nonthermal filaments appear to lie at eastern edge of the Sgr C cavity and the H II region. Like other filaments described in the Sgr A and Sgr B clouds, the prominent nonthermal filament appears to terminate at the Sgr C IRDC.

$G 359.30+0.033(\mathrm{~g} 30)$. This $4.5 \mu \mathrm{m}$ excess source is detected at the edge of an elongated and narrow IRDC at $l=40^{\prime}, b=2^{\prime}$. This feature appears to widen in the direction away from the Galactic plane, resembling the vertical molecular Clumps 1 and 2 that are found at $l=355^{\circ}$ and $l=3^{\circ}$ and $b=0^{\circ}$ (Bania 1977). Figures 23(a) and (b) show an $8 \mu \mathrm{m}$ and $24 \mu \mathrm{m}$ images of this remarkable filamentary dark cloud. The cloud is drawn schematically in Figure 1(c). The brightness of this cloud in the ratio map shown in Figure 3(b) suggests that it is likely to be on the front side of the Galactic center. Contours of $4.5 \mu \mathrm{m}$ emission from the region where excess emission is detected at this wavelength are shown in Figure 23(c). The fit to the SED of this source suggests a $8.9 M_{\odot}$ protostar (see Table 9).

G359.199+0.041 (g31). This $4.5 \mu \mathrm{m}$ excess source is projected against an east-west elongated IRDC. Figures 24(a) and (b) show $24 \mu \mathrm{m}$ and $20 \mathrm{~cm}$ continuum images of this source. A methanol maser source and a $20 \mathrm{~cm}$ continuum source lie in its vicinity, $4^{\prime}$ away from the $4.5 \mu \mathrm{m}$ excess source. If the methanol source is associated with the same IRDC toward which 

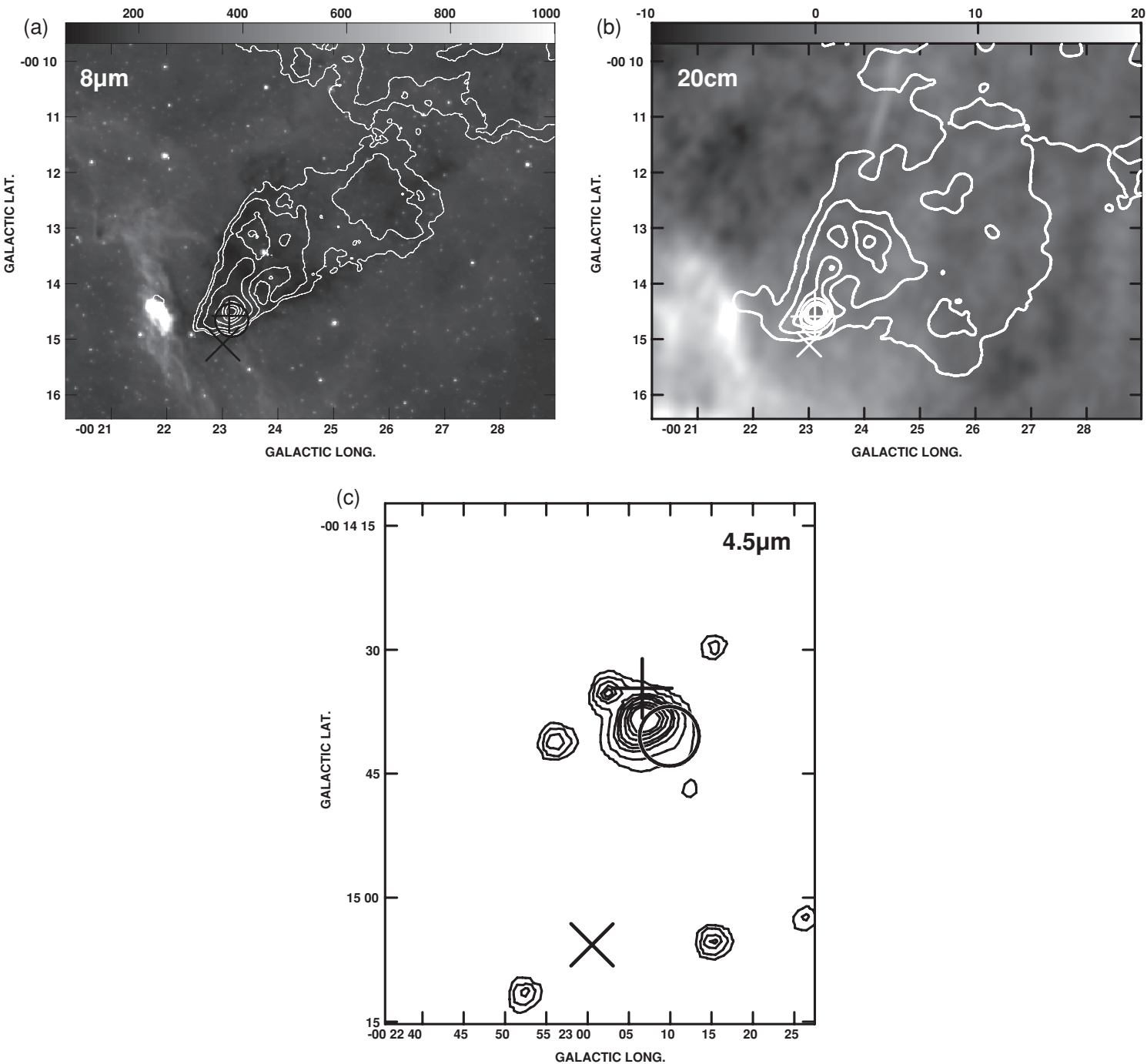

Figure 34. (a) Contours of $450 \mu \mathrm{m}$ emission from g26 at 3, 4.5, 8, 15, 25, 40, and $60 \mathrm{Jy}^{-1}$ beam $^{-1}$ are superimposed on a $8 \mu \mathrm{m}$ continuum image. (b) Contours of $850 \mu \mathrm{m}$ emission from g26 at 2, 3, 4, 5, 7, and $9 \mathrm{Jy} \mathrm{beam}^{-1}$ are superimposed on a gray-scale $20 \mathrm{~cm}$ continuum image. (c) Contours of $4.5 \mu \mathrm{m}$ emission from g26 is shown at 50, 100, 200, 300, 400, 600, 800, and $1000 \mathrm{MJy} \mathrm{sr}^{-1}$. Symbols are as defined in Figure 17.

the $4.5 \mu \mathrm{m}$ excess source is detected, then the IRDC is a site of massive star formation. Contours of $4.5 \mu \mathrm{m}$ emission from this cloud are shown in Figure 24(c). The fit to the SED of this source suggests a $14.4 M_{\odot}$ protostar (see Table 9). However, the SED is not fitted well by the models because of the narrow peak at mid-IR wavelengths.

$G 358.980+0.084(\mathrm{~g} 32)$. The $4.5 \mu \mathrm{m}$ excess source 32 is projected at the center of an IRDC which has a spidery appearance. Figures 25(a) and (b) show $24 \mu \mathrm{m}$ and $8 \mu \mathrm{m}$ images of this source. There is no evidence of a compact radio counterpart at $20 \mathrm{~cm}$ to the $4.5 \mu \mathrm{m}$ excess source, though, a compact source is detected about $1^{\prime}$ southwest of it. The methanol maser coincident with the position of the $4.5 \mu \mathrm{m}$ excess source supports the presence of on-going massive star formation in this dark cloud. The SED fit to the source identified by its excess $4.5 \mu \mathrm{m}$ emission gives a $7.6 M_{\odot}$ protostar. Figure 25(d) presents contours of $4.5 \mu \mathrm{m}$ emission from this source.

\section{A.2. Foreground $4.5 \mu \mathrm{m}$ Excess Sources}

Figure 15(b) and Table 10 show the SEDs of individual foreground sources and list the physical characteristics derived from the fits, respectively. The estimated $A_{v}$ range between 4 and 33 mag. Due to the distance uncertainty, the mass estimate is not well constrained. We note that with the exception of three sources, all the $4.5 \mu \mathrm{m}$ excess sources appear to be extended. In addition, with the exception of one, all the SED fitted sources appear to be in Stage I suggesting their early evolutionary phase.

$0.955-0.786(g 1), 0.868-0.697(g 2)$, and $0.780-0.740(g 4)$. All the three $4.5 \mu \mathrm{m}$ excess sources g1, g2, and g4 are located at the edge of the region surveyed with IRAC. Sources g1, g4, and g2 coincide with the positions of three PNe JaST 76 and JaST 70 in Van de Steene \& Jacoby (2001) and PN G0.8-0.6 in Kerber et al. (2003), respectively. The SEDs of these sources are shown in Figure 16(b). The successful fitting of these PNe shows that SED fitting cannot be used to distinguish them from other source types. Additional compact $4.5 \mu \mathrm{m}$ excess sources may be PNe that are misidentified as YSOs.

$G 0.708+0.408(g 5)$. This source is projected against an IRDC observed with the MIPS and IRAC data. Figures 26(a) and (b) show a $24 \mu \mathrm{m}$ image and a $4.5 \mu \mathrm{m}$ contour of the $4.5 \mu \mathrm{m}$ excess source. The SED fit to this source suggests a $6 M_{\odot}$ protostar (see Table 10) as shown in Figure 16(b). However, the SED is not fit well because of its narrow peak. 

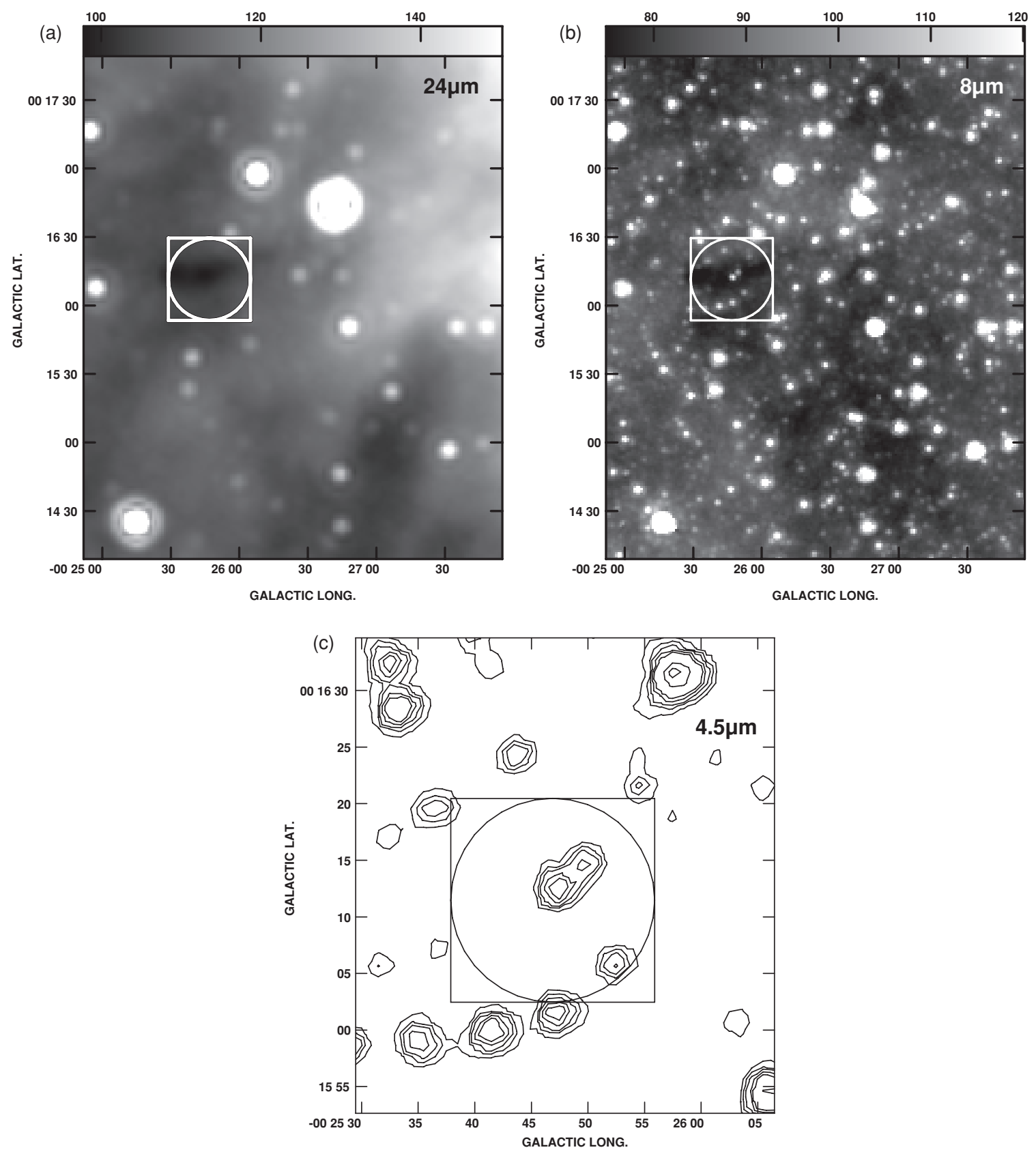

Figure 35. (a) $24 \mu \mathrm{m}$ gray-scale image of the region in the vicinity of g28 which lies at the center of the circle and square symbols. The flux range is between 98 and $150 \mathrm{MJy} \mathrm{sr}^{-1}$. (b) Similar to (a) except that the emission is seen at $8 \mu \mathrm{m}$. The flux range is between 75 and $120 \mathrm{MJy} \mathrm{sr}^{-1}$ (c) Contour levels of emission at $4.5 \mu \mathrm{m}$ are set at 25, 35, 50, 75, 100, 300, and $500 \mathrm{MJy} \mathrm{sr}^{-1}$. Symbols are as defined in Figure 17.

$4.5 \mu \mathrm{m}$ Excess Sources Associated with Sh20 (g11-g15, g12g14). Several $4.5 \mu \mathrm{m}$ excess sources associated with Sh20 are distributed at negative latitudes where several prominent optical emission nebulae have been identified. The $4.5 \mu \mathrm{m}$ excess sources g11, g12, g13, g14, and g15 are most likely associated with Sh20 (Sharpless 20) centered at $l=0.5, b=-0.3$ with an extent of 10' (Mar Vsálková 1974). The SED fitting to these $4.5 \mu \mathrm{m}$ excess sources give masses in the range between 2 and $14 M_{\odot}$ (see Table 10).

Figures 27(a) and (b) show $24 \mu \mathrm{m}$ and $8 \mu \mathrm{m}$ images of G0.542-0.476 where $4.5 \mu \mathrm{m}$ excess source g11 is detected. The $4.5 \mu \mathrm{m}$ excess source appears to be projected against a dearth of emission at $8 \mu \mathrm{m}$ in the vicinity of an elongated IRDC with an extent of $2^{\prime}$. A sharp linear feature with an extent of $\sim 1^{\prime}$ lies to the north. The lack of a counterpart at
$24 \mu \mathrm{m}$ and/or at radio wavelengths suggests that this linear feature is likely to arise from PAH emission. Figure 27(c) shows a radio continuum image of the region where g11 is detected and Figure 27(d) shows a $4.5 \mu \mathrm{m}$ contour map of $\mathrm{g} 11$.

Other $4.5 \mu \mathrm{m}$ excess sources that appear to be associated with Sharpless 20 are G0.517-0.657 (g12), G0.483-0.701 (g13), and G0.477-0.727 (g14). Figures 28(a)-(c) show $24 \mu \mathrm{m}, 8 \mu \mathrm{m}$, and $20 \mathrm{~cm}$ gray-scale images of the region where these $4.5 \mu \mathrm{m}$ excess sources are detected. The $4.5 \mu \mathrm{m}$ emission contours for g12, g13, and g14 are shown in Figures 28(d)-(f), respectively. It is clear that this is a region of massive star formation where sharp extended shell-like structures and a compact radio continuum source coincident with g14 are seen at $24 \mu \mathrm{m}$ and $20 \mathrm{~cm}$. No $8 \mu \mathrm{m}$ counterparts are found. 
Another H II structure with the appearance of a "question mark" is a component of Sharpless 20. The $4.5 \mu \mathrm{m}$ excess source g15 lies at the center of this H II region. Figures 29(a)(c) show gray-scale images at $24 \mu \mathrm{m}, 8 \mu \mathrm{m}$, and $20 \mathrm{~cm}$, respectively, where as a contour map of g15 at $4.5 \mu \mathrm{m}$ is shown in Figure 29(d).

G0.315-0.21 (g17). This $4.5 \mu \mathrm{m}$ excess source lies in the vicinity of two stellar cluster candidates that are located within 1 arcmin of each other and within the Sh2-20 HII region (Sharpless 1959; Dutra et al. 2003). This cluster also has variable $\mathrm{X}$-ray emission, indicating very young stars (Law \& YusefZadeh 2004). Figures 30(a) and (b) show contours at $450 \mu \mathrm{m}$ and $850 \mu \mathrm{m}$ superimposed on a gray-scale $24 \mu \mathrm{m}$ and $20 \mathrm{~cm}$ images of this $\mathrm{H}$ II region. Two sources in the $24 \mu \mathrm{m}$ and $20 \mathrm{~cm}$ images lie in the vicinity of a submillimeter peak. Contours of $4.5 \mu \mathrm{m}$ emission from both the northern and southern sources are shown in Figure 30(c). The $4.5 \mu \mathrm{m}$ excess source coincides with the southern component of the emission shown in these figures. The presence of methanol masers coincident with the $4.5 \mu \mathrm{m}$ excess source and a bright submillimeter peak provide strong evidence for massive star formation in this region. The fit to the SED of the $4.5 \mu \mathrm{m}$ excess source is also consistent with $12.8 M_{\odot}$ protostar.

G0.167-0.445 (g18), G0.091-0.663 (g19), and G0.084$0.642(\mathrm{~g} 20)$. The $4.5 \mu \mathrm{m}$ excess sources $\mathrm{g} 18, \mathrm{~g} 19$, and $\mathrm{g} 20$ are projected toward the H II region RCW 141, which is centered near $l=0.3, b=-0.2$ with an extent of $6^{\prime} \times 4^{\prime}$. Figures 31 (a) and (b) show $8 \mu \mathrm{m}$ and $20 \mathrm{~cm}$ gray-scale images of $\mathrm{g} 18$, respectively. The two $8 \mu \mathrm{m}$ sources appear to be embedded within an IRDC. However, the one indicated by a cross shows strong excess emission at $4.5 \mu \mathrm{m}$. A nonthermal radio filament that runs perpendicular to the Galactic plane is projected against the $4.5 \mu \mathrm{m}$ excess source. Figures 32(a) and (b) show grayscale $24 \mu \mathrm{m}$ and $8 \mu \mathrm{m}$ images of g19 and g20, respectively. It is clear that the southern source $\mathrm{g} 19$ is embedded in an IRDC. Figure 32(c) shows that neither of these $4.5 \mu \mathrm{m}$ excess sources has $20 \mathrm{~cm}$ continuum counterparts. Figures 32(d) and (e) show contours of $4.5 \mu \mathrm{m}$ emission from g19 and g20, respectively.

$6359.939+0.170(g 22)$. The $4.5 \mu \mathrm{m}$ excess source $\mathrm{g} 22$ is located in an H II complex with an IRDC and a prominent $8 \mu \mathrm{m}$ nebulosity. Figures 33(a) and (b) show contours of $850 \mu \mathrm{m}$ emission superimposed on gray-scale images at $24 \mu \mathrm{m}$ and $20 \mathrm{~cm}$, respectively. The $850 \mu \mathrm{m}$ emission and the IRDC at $24 \mu \mathrm{m}$ appear to lie at the edge of the nonthermal filament, as seen in Figure 33(b). A large-scale view of the dark cloud and the extensive $8 \mu \mathrm{m}$ emission to its eastern edge are shown in Figure 33(c). Contours of the source responsible for excess $4.5 \mu \mathrm{m}$ emission are shown in Figure 33(d).

G359.972-0.459 (g21), G359.907-0.303 (g24), and G359.618-0.245 (g26). The $4.5 \mu \mathrm{m}$ excess sources g21, g24, and g26 are distributed in the vicinity of RCW 137 which is roughly centered close to $l=0.1, b=-0.3$ with an extent of $2^{\prime} \times 2^{\prime}$. The most interesting of these $4.5 \mu \mathrm{m}$ excess sources is $\mathrm{g} 26$, which is embedded in a prominent submillimeter core tracing an IRDC. Figures 34(a) and (b) show contours of $450 \mu \mathrm{m}$ and $850 \mu \mathrm{m}$ emission superimposed on $8 \mu \mathrm{m}$ and $20 \mathrm{~cm}$ gray-scale images of the IRDC associated with g26. The submillimeter cone-shaped structure peaks where methanol maser emission is detected. The closeness of methanol masers, the $4.5 \mu \mathrm{m}$ excess source and the submillimeter core support the evidence for massive star formation in this cloud. Figure 34(c) shows contours of $4.5 \mu \mathrm{m}$ emission from $\mathrm{g} 26$, which has an estimated $7 M_{\odot}$ protostar (see Table 10).
G359.57-0.27 $(g 28)$. The $4.5 \mu \mathrm{m}$ excess source g28 lies at the edge of an IRDC that extends for $2^{\prime}$. Images of the region at 24 and $8 \mu \mathrm{m}$ are shown in Figures 35(a) and (b). g28 is located in the darkest area of the IR dark cloud, which shows an eastwest elongation. The source was also detected at $70 \mu \mathrm{m}$, which allows us to constrain the mass of the YSO candidate to be $4 M_{\odot}$ assuming an $\left\langle A_{v}\right\rangle=4$. Contours of $4.5 \mu \mathrm{m}$ emission are shown Figure 35(c).

\section{REFERENCES}

Adams, F. C., Lada, C. J., \& Shu, F. H. 1987, ApJ, 312, 788

Arendt, R. G., et al. 2008, ApJ, 682, 384

Argon, A. L., Reid, M. J., \& Menten, K. M. 2000, ApJS, 129, 159

Armstrong, J. T., \& Barrett, A. H. 1985, ApJS, 57, 535

Bally, J., Stark, A. A., Wilson, R. W., \& Henkel, C. 1988, ApJ, 324, 223

Bania, T. M. 1977, ApJ, 216, 381

Becker, R. H., White, R. L., Helfand, D. J., \& Zoonematkermani, S. 1994, ApJS, 91, 347

Beuther, H., Churchwell, E. B., McKee, C. F., \& Tan, J. C. 2007, in Protostars and Planets V, ed. B. Reipurth, D. Jewitt, \& K. Keil (Tucson, AZ: Univ. Arizona Press), 165

Beuther, H., Sridharan, T. K., \& Saito, M. 2005, ApJ, 634, L185

Binney, J., Gerhard, O. E., Stark, A. A., Bally, J., \& Uchida, K. I. 1991, MNRAS, 252,210

Carey, S. J., Feldman, P. A., Redman, R. O., Egan, M. P., MacLeod, J. M., \& Price, S. D. 2000, ApJ, 543, L157

Carey, S. J., et al. 2009, PASP, 121, 76

Caswell, J. L. 1996, MNRAS, 283, 606

Chambers, E. T., Jackson, J. M., Rathborne, J. M., \& Simon, R. 2009, ApJS, 181,360

Chapman, N. L., Mundy, L. G., Lai, S.-P., \& Evans, N. J. 2009, ApJ, 690, 496

Churchwell, E., et al. 2007, ApJ, 670, 428

Condon, J. J. 1992, ARA\&A, 30, 575

Cox, P., \& Laureijs, R. 1989, in IAU Symp., Vol. 136, The Center of the Galaxy, ed. M. Morris (Dordrecht: Kluwer), 121

Cyganowski, C. J., et al. 2008, AJ, 136, 2391

De Pree, C. G., Wilner, D. J., Deblasio, J., Mercer, A. J., \& Davis, L. E. 2005, ApJ, 624, L101

Di Francesco, J., Johnstone, D., Kirk, H., MacKenzie, T., \& Ledwosinska, E. 2008, ApJS, 175, 277

Dutra, C. M., Ortolani, S., Bica, E., Barbuy, B., Zoccali, M., \& Momany, Y. 2003, A\&A, 408, 127

Egan, M. P., Shipman, R. F., Price, S. D., Carey, S. J., Clark, F. O., \& Cohen, M. 1998, ApJ, 494, L199

Felli, M., Comoretto, G., Testi, L., Omont, A., \& Schuller, F. 2000, A\&A, 362, 199

Figer, D. F., Kim, S. S., Morris, M., Serabyn, E., Rich, R. M., \& McLean, I. S. 1999, ApJ, 525, 750

Flaherty, K. M., Pipher, J. L., Megeath, S. T., Winston, E. M., Gutermuth, R. A., Muzerolle, J., Allen, L. E., \& Fazio, G. G. 2007, ApJ, 663, 1069

Fuchs, B., Jahreiß, H., \& Flynn, C. 2009, AJ, 137, 266

Fuerst, E., Reich, W., \& Sofue, Y. 1987, A\&AS, 71, 63

Gaume, R. A., Claussen, M. J., de Pree, C. G., Goss, W. M., \& Mehringer, D. M. 1995, ApJ, 449, 663

Ghez, A. M., et al. 2008, ApJ, 689, 1044

Gordon, K. D., et al. 2007, PASP, 119, 1019

Güsten, R., \& Philipp, S. D. 2004, in The Dense Interstellar Medium in Galaxies, ed. S. Pfalzner et al. (Berlin: Springer), 253

Habing, H. J. 1996, A\&AR, 7, 97

Haller, J. W., Rieke, M. J., Rieke, G. H., Tamblyn, P., Close, L., \& Melia, F. 1996, ApJ, 456, 194

Haschick, A. D., Menten, K. M., \& Baan, W. A. 1990, ApJ, 354, 556

Helou, G., \& Bicay, M. D. 1993, ApJ, 415, 93

Herrnstein, R. M., \& Ho, P. T. P. 2005, ApJ, 620, 287

Hinz, J. L., Rieke, G. H., Yusef-Zadeh, F., Hewitt, J., Balog, Z., \& Block, M. 2009, ApJS, 181, 227

Hüttemeister, S., Wilson, T. L., Bania, T. M., \& Martin-Pintado, J. 1993, A\&A, 280,255

Jacoby, G. H., \& Van de Steene, G. 2004, A\&A, 419, 563

Kennicutt, R. C., Jr. 1998, ARA\&A, 36, 189

Jones, P. A., et al. 2008, MNRAS, 386, 117

Kerber, F., Mignani, R. P., Guglielmetti, F., \& Wicenec, A. 2003, A\&A, 408, 1029 
Kroupa, P. 2001, MNRAS, 322, 231

Lada, C. J. 1987, in Proc. IAU Symp. 115, Star Forming Regions, ed. M. Peimbert \& J. Jugaku (Dordrecht: Reidel), 1

Lada, C. J. 1999, in NATO ASIC Proc. 540, The Origin of Stars and Planetary Systems, ed. C. J. Lada \& N. D. Kylafis (Dordrecht: Kluwer), 143

Lang, C. C., Morris, M., \& Echevarria, L. 1999, ApJ, 526, 727

Law, C., \& Yusef-Zadeh, F. 2004, ApJ, 611, 858

Law, C. J., Yusef-Zadeh, F., Cotton, W. D., \& Maddalena, R. J. 2008, ApJS, 177,255

Lee, J.-K., Walsh, A. J., Burton, M. G., \& Ashley, M. C. B. 2001, MNRAS, 324,1102

Li, A., \& Draine, B. T. 2001, ApJ, 554, 778

Lindqvist, M., Habing, H. J., \& Winnberg, A. 1992, A\&A, 259, 118

Lis, D. C., \& Carlstrom, J. E. 1994, ApJ, 424, 189

Lis, D. C., Serabyn, E., Zylka, R., \& Li, Y. 2001, ApJ, 550, 761

Lis, D. C., \& Menten, K. M. 1998, ApJ, 507, 794

Liszt, H. S. 1992, ApJS, 82, 495

Liszt, H. S., \& Spiker, R. W. 1995, ApJS, 98, 259

Loose, H. H., Kruegel, E., \& Tutukov, A. 1982, A\&A, 105, 342

Lu, J. R., Ghez, A. M., Hornstein, S. D., Morris, M., Matthews, K., Thompson, D. J., \& Becklin, E. E. 2006, J. Phys. Conf. Ser., 54, 279

Marston, A. P., et al. 2004, ApJS, 154, 333

Martin, C. L., Walsh, W. M., Xiao, K., Lane, A. P., Walker, C. K., \& Stark, A. A. 2004, ApJS, 150, 239

Mar Vsálková, P. 1974, Ap\&SS, 27, 3

Mehringer, D. M., Goss, W. M., Lis, D. C., Palmer, P., \& Menten, K. M. 1998, ApJ, 493, 274

Mehringer, D. M., \& Menten, K. M. 1997, ApJ, 474, 346

Menten, K. M. 1991, ApJ, 380, L75

Morris, M., \& Serabyn, E. 1996, ARA\&A, 34, 645

Morris, M., Uchida, K., \& Do, T. 2006, Nature, 440, 308

Murphy, E. J., et al. 2006, ApJ, 638, 157

Nord, M. E., Lazio, T. J. W., Kassim, N. E., Hyman, S. D., LaRosa, T. N., Brogan, C. L., \& Duric, N. 2004, AJ, 128, 1646

Noriega-Crespo, A., Moro-Martin, A., Carey, S., Morris, P. W., Padgett, D. L., Latter, W. B., \& Muzerolle, J. 2004, ApJS, 154, 402

Odenwald, S. F., \& Fazio, G. G. 1984, ApJ, 283, 601

Ojha, D. K., Tej, A., Schultheis, M., Omont, A., \& Schuller, F. 2007, MNRAS, 381,1219

Oka, T., Geballe, T. R., Goto, M., Usuda, T., \& McCall, B. J. 2005, ApJ, 632, 882

Oka, T., Hasegawa, T., Sato, F., Tsuboi, M., \& Miyazaki, A. 1998, ApJS, 118 , 455

Omont, A., et al. 2003, A\&A, 403, 975

Ortiz, R., et al. 2002, A\&A, 388, 279

Panagia, N. 1973, AJ, 78, 929

Paumard, T., et al. 2006, ApJ, 643, 1011

Pierce-Price, D., et al. 2000, ApJ, 545, L121

Povich, M. S., et al. 2007, ApJ, 660, 346

Ramirez, S. V., Arendt, R. G., Sellgren, K., Stolovy, S. R., Cotera, A., Smith, H. A., \& Yusef-Zadeh, F. 2008, ApJS, 175, 147

Rathborne, J. M., Jackson, J. M., Chambers, E. T., Simon, R., Shipman, R., \& Frieswijk, W. 2005, ApJ, 630, L181

Reach, W. T., et al. 2006, AJ, 131, 1479

Rieke, G. H., Alonso-Herrero, A., Weiner, B. J., Pérez-González, P. G., Blaylock, M., Donley, J. L., \& Marcillac, D. 2009, ApJ, 692, 556
Robitaille, T. P., Whitney, B. A., Indebetouw, R., \& Wood, K. 2007, ApJS, 169, 328

Robitaille, T. P., Whitney, B. A., Indebetouw, R., Wood, K., \& Denzmore, P. 2006, ApJS, 167, 256

Schuller, F., Omont, A., Glass, I. S., Schultheis, M., Egan, M. P., \& Price, S. D. 2006, A\&A, 453, 535

Sevenster, M. N., Chapman, J. M., Habing, H. J., Killeen, N. E. B., \& Lindqvist, M. 1997a, A\&AS, 122, 79

Sevenster, M. N., Chapman, J. M., Habing, H. J., Killeen, N. E. B., \& Lindqvist, M. 1997b, A\&AS, 124, 509

Sharpless, S. 1959, ApJS, 4, 257

Shiki, S., Ohishi, M., \& Deguchi, S. 1997, ApJ, 478, 206

Simon, R., Rathborne, J. M., Shah, R. Y., Jackson, J. M., \& Chambers, E. T. 2006, ApJ, 653, 1325

Sjouwerman, L. O., van Langevelde, H. J., Winnberg, A., \& Habing, H. J. 1998, A\&AS, 128, 35

Skrutskie, M. F., et al. 1997, in Astrophysics and Space Science Library 210, The Impact of Large Scale Near IR Sky Surveys, ed. F. Garzon et al. (Dordrecht: Kluwer), 25

Slysh, V. I., Kalenskii, S. V., Val'tts, I. E., \& Otrupcek, R. 1994, MNRAS, 268, 464

Smith, H. A., Hora, J. L., Marengo, M., \& Pipher, J. L. 2006, ApJ, 645, 1264

Stark, A. A., Bally, J., Gerhard, O. E., \& Binney, J. 1991, MNRAS, 248, 14P

Stolovy, S., et al. 2006, J. Phys. Conf. Ser., 54, 176

Taylor, G. B., Morris, M., \& Schulman, E. 1993, AJ, 106, 1978

Tilley, D. A., Balsara, D. S., \& Howk, J. C. 2006, MNRAS, 371, 1106

Tsuboi, M., Handa, T., \& Ukita, N. 1999, ApJS, 120, 1

Tutukov, A., \& Kruegel, E. 1978, A\&A, 67, 437

Uchida, K., Morris, M., \& Serabyn, E. 1990, ApJ, 351, 443

Vacca, W. D., Garmany, C. D., \& Shull, J. M. 1996, ApJ, 460, 914

Val'tts, I. E., Ellingsen, S. P., Slysh, V. I., Kalenskii, S. V., Otrupcek, R., \& Larionov, G. M. 2000, MNRAS, 317, 315

Van de Steene, G. C., \& Jacoby, G. H. 2001, A\&A, 373, 536

Walsh, A. J., Burton, M. G., Hyland, A. R., \& Robinson, G. 1998, MNRAS, 301,640

Wardle, M., \& Yusef-Zadeh, F. 2008, ApJ, 683, L37

Watson, C., et al. 2008, ApJ, 681, 1341

Whitney, B. A., Wood, K., Bjorkman, J. E., \& Cohen, M. 2003a, ApJ, 598, 1079

Whitney, B. A., Wood, K., Bjorkman, J. E., \& Wolff, M. J. 2003b, ApJ, 591, 1049

Whitney, B. A., et al. 2008, AJ, 136, 18

Yusef-Zadeh, F., et al. 2007a, in IAU Symp. Vol. 242, Masers as Probes of Massive Star Formation in the Nuclear Disk, ed. J. M. Chapman \& W. A. Baan (Drodrecht: Kluwer), 366

Yusef-Zadeh, F., Muno, M., Wardle, M., \& Lis, D. C. 2007b, ApJ, 656, 847

Yusef-Zadeh, F., Goss, W. M., Roberts, D. A., Robinson, B., \& Frail, D. A. 1999, ApJ, 527, 172

Yusef-Zadeh, F., Hewitt, J. W., \& Cotton, W. 2004, ApJS, 155, 421

Yusef-Zadeh, F., \& Morris, M. 1987, AJ, 94, 1178

Yusef-Zadeh, F., Morris, M., \& Chance, D. 1984, Nature, 310, 557

Yusef-Zadeh, F., Wardle, M., Muno, M., Law, C., \& Pound, M. 2005, Adv. Space Res., 35, 1074

Zhao, J.-H., Desai, K., Goss, W. M., \& Yusef-Zadeh, F. 1993, ApJ, 418, 235

Zoonematkermani, S., Helfand, D. J., Becker, R. H., White, R. L., \& Perley, R. A. 1990, ApJS, 74, 181

Zylka, R., Mezger, P. G., \& Wink, J. E. 1990, A\&A, 234, 133 